Nikolai Kislov (i https://orcid.org/0000-0003-2129-3896

Nano CVD Company, Tampa, Florida, 33626, USA

E-mail: nikolai95@verizon.net

\title{
Physical Approach to Solving the Mathematical Navier-Stokes Problem
}

\begin{abstract}
Instead of modeling based on using an infinitesimal fluid element, which is treated as a continuous medium, we consider fluid flow in a fluid system as a model gas flow in a model gas system identical to the fluid system. We assigned to the model gas properties, which differ from the properties typically assigned to the ideal gas. In our approach, we mimic the movement of each particle/molecule composing the model gas and then gather that movement into macro quantities characterizing the fluid flow. We formulated integro-differential balance equations for mass, momentum, and energy applied to any non-moving point in three-dimensional space occupied by the model gas and operable from the continuum through the rarefied to the ballistic flow regimes. In parallel, we worked toward understanding how our derived integro-differential equations of the balance may relate to the existing Navier-Stokes equations. Since Navier-Stokes equations are limited to the continuum and collisiondominated flow regimes, we reduced the derived mass and momentum integro-differential balance equations by if during the period between sequential collisions, the relative change of any property value or any parameter characterizing the model gas is insignificant. Then by applying the method of vector differentiation, we converted the mass and momentum integro-differential balance equations into corresponding vector differential balance equations. We were surprised to observe that our converted differential forms look identically to the Navier-Stokes equations. This finding has led us to the conclusion that, in the collision-dominated flow regime, the formulated integro-differential forms of the balance are exact implicit solutions for corresponding Navier-Stokes equations. This finding also suggests that the proposed matching vector integro-differential forms of the mass, momentum, and energy balance, which can be solved by computer-implemented methods with no difficulty, may represent a physical problem described by the Navier-Stokes equations. We also provided five additional validation tests demonstrating the feasibility of the proposed method.
\end{abstract}

Keywords: Navier, gas, particle, trajectory, balance, fluid, flow

\section{Introduction}

Computational Fluid Dynamics (CFD) is widely used in many practical applications ranging from basic hydrodynamic and kinematic to fundamental cosmological applications. The fundamental basis of any CFD tool is a solver, which solves the Navier-Stokes equations that are a set of second-order partial differential equations. Navier-Stokes equations are based on the assumption that the fluid is a continuum, and they are derived by considering the mass, momentum, and energy conservations for a control volume of any size. The flow is considered continuous and differentiable, allowing the mass, momentum, and energy balances to be expressed as partial differential equations. Scientists and engineers made further approximations and simplifications to the Navier-Stokes equation set until it can be solved [1]. However, this intentional simplification of a fluid model may diminish the usefulness of the results of the computations. Also, the theoretical understanding of the solutions to these equations is still inadequate. Specifically, for three-dimensional Navier-Stokes equations and 
given initial conditions, mathematicians have not yet proved that smooth solutions always exist, and the solutions have limited energy per unit mass. The complete solution of the NavierStokes equations or at least proof of the existence of smooth solutions to the form of the NavierStokes equations has remained to be one of the principal unsolved problems in mathematics for almost two centuries [2]. In the most general form, the Navier-Stokes equations of mass and momentum conservation for compressible fluid are expressed as:

$$
\frac{\partial}{\partial t} \rho+\boldsymbol{\nabla} \cdot(\rho \overrightarrow{\boldsymbol{u}})=0
$$

and

$$
\frac{\partial}{\partial t} \overrightarrow{\boldsymbol{u}}(t, \overrightarrow{\boldsymbol{r}})+(\overrightarrow{\boldsymbol{u}} \cdot \boldsymbol{\nabla}) \overrightarrow{\boldsymbol{u}}(t, \overrightarrow{\boldsymbol{r}})+\frac{1}{\rho} \boldsymbol{\nabla} p(t, \overrightarrow{\boldsymbol{r}})-\overrightarrow{\boldsymbol{g}}(t, \overrightarrow{\boldsymbol{r}})=\frac{1}{\rho} \boldsymbol{\nabla} \cdot \mathrm{T},
$$

respectively, for $t>0$ and $\overrightarrow{\boldsymbol{r}} \in \mathbb{R}^{\mathbf{3}}$. In the equations above, $\rho$ is the density of the fluid, $\overrightarrow{\boldsymbol{u}}$ is mass flow velocity, $\boldsymbol{\nabla} p$ is the pressure gradient, $\boldsymbol{\nabla} \cdot \mathrm{T}$ represents stresses inside the fluid, and $\overrightarrow{\boldsymbol{g}}$ is the external force per unit mass. The right-hand term $\boldsymbol{\nabla} \cdot \mathrm{T}$ has generally unknown functionality and contains too many unknowns and up to date is not applicable to practical problems.

The Navier-Stokes equations for an incompressible Newtonian fluid in Fefferman's formulation [3] and in the interpretation close to Lemarie-Rieusset [2] are shown in a vectordifferential form:

$$
\frac{\partial}{\partial t} \overrightarrow{\boldsymbol{u}}(t, \overrightarrow{\boldsymbol{r}})+(\overrightarrow{\boldsymbol{u}} \cdot \nabla) \overrightarrow{\boldsymbol{u}}(t, \overrightarrow{\boldsymbol{r}})+\frac{1}{\rho} \nabla p(t, \overrightarrow{\boldsymbol{r}})-\overrightarrow{\boldsymbol{g}}(t, \overrightarrow{\boldsymbol{r}})=\frac{1}{\rho} \mu \Delta \overrightarrow{\boldsymbol{u}}(t, \overrightarrow{\boldsymbol{r}})
$$

for $t>0$ and $\overrightarrow{\boldsymbol{r}} \in \mathbb{R}^{3}$

$$
\boldsymbol{\nabla} \cdot \overrightarrow{\boldsymbol{u}}=0
$$

with initial condition

$$
\overrightarrow{\boldsymbol{u}}(0, \overrightarrow{\boldsymbol{r}})=\overrightarrow{\boldsymbol{u}}_{\mathbf{0}}(\overrightarrow{\boldsymbol{r}}) \text { for } \quad \overrightarrow{\boldsymbol{r}} \in \mathbb{R}^{\mathbf{3}},
$$

where $\mu \Delta \overrightarrow{\boldsymbol{u}}(t, \overrightarrow{\boldsymbol{r}})$ is a viscous force, $\mu$ is the viscosity, $\Delta$ is the Laplacian, and unknowns are the vector velocity of the fluid element $\overrightarrow{\boldsymbol{u}}(t, \overrightarrow{\boldsymbol{r}})$ and the pressure exerted on the fluid element $p(t, \overrightarrow{\boldsymbol{r}})$. The equations describe the motion of a fluid filling the whole space $\mathbb{R}^{3}$. The fluid is assumed to be homogeneous. Given the initial state $\overrightarrow{\boldsymbol{u}}_{\mathbf{0}}(\overrightarrow{\boldsymbol{r}})$ at time $t=0$ and acceleration $\overrightarrow{\boldsymbol{g}}$ for $t>0$, one wants to determine the evolution of the system for $t>0$ [2]. However, according to Fefferman [3], a "fundamental problem in analysis is to decide whether such smooth, physically reasonable solutions exist for the Navier-Stokes equations." Tao [4] admits that "the long-time evolution of a dynamical system for deterministic data is still largely only controllable by the classical tools of exact solutions, conservation laws and monotonicity formulae; a discovery of a new and effective tool for this purpose would be a major breakthrough." In the concluding remarks of the official statement of the Navier-Stokes existence and smoothness problem, Fefferman [3] wrote: "Since we don't even know whether these solutions exist, our understanding is at a very primitive level. Standard methods from PDE appear inadequate to settle the problem. Instead, we probably need some deep, new ideas."

The general major disadvantage of any existing "mathematical" approach in solving the fluid dynamics problem consists of using an infinitesimal fluid element viewed as a continuous 
medium, to which fundamental physical principles are applied. This approach contradicts the molecular or particle nature, thus providing a source of significant uncertainty in interpreting the results of modeling and calculations. In small-scale flows, when the characteristic hydrodynamic length scale approaches the fluid characteristic length scale, the Navier-Stokes description is expected to fail (see references of Karnidakis and Beskok [5] and Gad-el-Hak [6]). In rarefied gases, the mean free path or the average distance traveled between intermolecular collisions is also considered a fail factor for the Navier-Stokes description [7].

Mathematicians and physicists consider the recent advancement of the method of the lattice Boltzmann (LB) equation as a serious alternative to standard computational fluid dynamics [8]. This approach consists in modeling "the physical reality at a mesoscopic level: the generic features of microscopic processes can be expressed through simple rules, from which the desired macroscopic behavior emerges as a collective effect of the interactions between the many elementary components [9]." Typical hydrodynamic quantities, such as mass, density, fluid velocity, and temperature, are not associated with individual particle movement and are quantified by simple moments of the particle distribution function [10]. However, solving the LB equation represents a significant challenge because it involves a numerical evaluation of an integral-differential equation in position and velocity phase space. Fluid simulation of such complexity can be performed effectively only by massively parallel data processors having combinational logic for processing collision rules. This method is enormously complicated and restricted because of the limitation of the available computational approaches for modeling real physical statistical systems. In addition, this method is not intended to solve the Navier-Stokes problem.

In this paper, we introduce a physical approach to finding implicit solutions for NavierStokes equations. The physical approach consists of approximating fluid flow as a flow of a model gas, assigning unique properties to the model gas, forming integro-differential balance equations for mass and momentum transport in any non-moving point in space occupied by the model gas, and, reducing these equations to corresponding differential balance equations. Here, in the interests of simplicity, unless otherwise stated, we limit our analysis to the Navier-Stokes problem in the three-dimensional space. In the patent publications [11] and [12], we disclosed detailed steps of the one-dimensional simulation of the model gas flow in a confined space between parallel plates, which support our method of modeling flows without using NavierStokes equations.

In the following, Section 2 describes the physical principles of the fluid model and the general physical approach of forming balancing equations. In Section 3, we provide integrodifferential forms of mass balance, momentum balance, and energy balance equations, which apply to any non-moving point in the space of the fluid at a given time. In Section 4, we validate the proposed approach by demonstrating that, in the collision-dominated flow regime, the differential equations, which were converted from the integro-differential mass balance and momentum balance equations, are identical to the corresponding Navier-Stokes equations In Section 5, we demonstrate five validation tests aimed to prove the feasibility of the proposed method. The first two examples, A1 and A2, illustrate that interaction of the model gas and the ideal gas with the gas-solid interface are identical (compare the rate of collision per unit area and the pressure exerted on the surface); nevertheless, some the most important properties 
characterizing the model gas and the ideal gas are different. In the third example, A.3.2, we directly validate our approach by formulating the mass-balance and the momentum-balance in one-dimensional incompressible gas space confined between two parallel plates at the uniform temperature.

In addition, in the examples A3 and A4, we explain a method for obtaining an analytical solution describing the incompressible model gas flow at the uniform temperature, which is confined in the space between two infinite parallel plates. Example A3 illustrates the model gas flow with diffuse particle scattering from the plates. Example A4 demonstrates the model gas flow in a case of mixed diffuse and specular particle scatterings from the plates being at rest.

Finally, in Section 6, we present the conclusions and highlight the prospectiveness of the proposed physical approach for developing a new generation of the CFD software that does not use the Navier-Stokes equations.

\section{Physical Principles of the Fluid Model}

We suggest an approach in which fluid flow in a fluid system is a model gas flow in a model gas system, which is identical to the fluid system. The transport processes involve the exchange of mass, momentum, energy, and other properties exchanged between interacting particles. In a more abstract sense, all particle interactions and property randomizing are an exchange of property/information [13]. We have also recognized that (1) transport phenomena include any situation that involves a net transfer of property/information between particles, which equals randomizing properties between interacting particles; (2) a randomized by property exchange between interacting particles physical/statistical property can be taken out into the surrounding gas [13]. However, we exclude interaction resulted in chemical reactions between colliding particles and fragmentation or fusion.

In the following, all references are made to absolute time, which is measured equally within the model gas system. Besides, when referencing an appropriate law of motion, Newton's Second Law of motion is considered. In addition, in the interests of simplicity, we analyzed the model gas flow at a uniform body force such as a gravitational field of force or the acceleration field if dealing with a particle of a unit mass.

\subsection{Properties and features of the model gas}

We assign these unique properties to the model gas:

(1) The model gas facilitates a distant transport of one or more properties, including one or more of mass, momentum, and energy by way of a plurality of particles being in a constant state of mostly random motion and interaction by collisions.

(2) Each of the plurality of particles of the model gas is assigned to travel by following a ballistic trajectory governed by a law of motion in free space. It overcomes a distance between any of two points the ballistic trajectory with certain survival probability, the ballistic trajectory having a starting point in one of a plurality of points of original collisions, and an ending point in one of a plurality of points of ending collisions. 
(3) Each of the plurality of particles is adapted to transport a combination of one or more properties, including one or more of mass, momentum, and energy between one of the plurality of points of original collisions and one of the plurality of points of ending collisions.

(4) Each point of the plurality of points in space occupied by the model gas is treated as a point of collisions for each of the plurality of particles following the ballistic trajectory with the same ending point simultaneously.

(5) Each of a plurality of the points of collisions is treated as either a point source for each of a plurality of diverging particles or a point sink for each of a plurality of converging particles.

(6) Each of the plurality of particles moving from the point source to the point sink is treated as a property carrier. The property carrier is created in the point source during the original collision by obtaining one or more properties of specific values being intrinsic to the model gas surrounding the point source. It is ended in the point sink during the ending collision by transferring one or more properties of specific values in the point sink.

(7) The value of the property, which is delivered in the point sink, or the value of the property, which is taken away from the point source, is evaluated regarding whether the value of the property is conserved or changed because of aging [11]. The value of the property is also evaluated for whether the value of property carried by each of the plurality of particles is modified because of interaction with an external field.

(8) The velocity of each of a plurality of point sources equals the mass flow velocity of the model gas in each of a plurality of corresponding points of the original collisions at the time of the original collision.

In this paper, in the interests of simplicity, we analyze the transport of properties not aged, which implies that the transported property is conserved during the ballistic traveling time.

Fig. 1 illustrates a schematic of ballistic traveling of a particle between two consecutive collisions in a fluid system composed of the model gas particles.

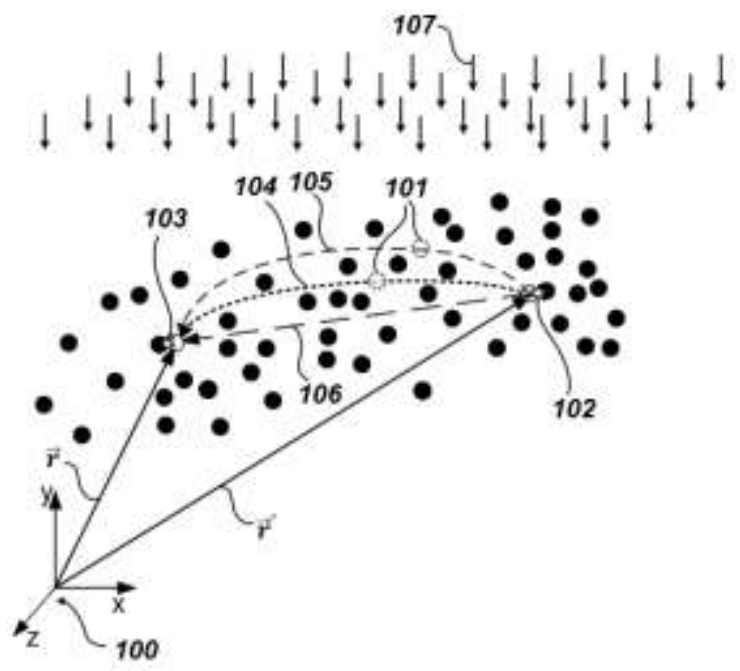

Fig. 1 Ballistic traveling of a particle between two consecutive collisions in a fluid system composed of the model gas particles 
The schematic diagram above shows the model gas composed of identical randomly moving particles and positioned in the observer's Cartesian coordinate system 100. Note that in the paper, the observer's coordinate system is designated by index "100." Here we consider an isotropic model, which requires that the coordinate system needs to be at rest. To locate the position of the event, the observer needs only to read the space coordinates at the location of an event. The clocks at any location within a system are synchronized. The observer, therefore, allocates space coordinates and time by recording both the space coordinates and time at the clock nearest the event position. For clarity, the observer's Cartesian coordinate system 100 is oriented, so y-axis is along the negative direction of an applied field of external force 107 which provides, for each of a plurality of particles, acceleration $\overrightarrow{\boldsymbol{g}}$. Each of the plurality of particles (shown as black disks), particularly a particle 101 (shown as transparent disks), travels in space between its two consecutive collisions: an original collision 102 and an ending collision 103 by following a trajectory 104 or 105 governed by an appropriate law of motion such as Newton's laws of motion. In a lack of external force, all ballistic trajectories between consecutive collisions will be just straight lines, respectively, as indicated by trajectory 106. Specifically, referring to Fig. 1, particle 101 obtains a set of properties of certain values in a point source 102 at the time of the original collision. The property may include a scalar property of value $\Psi$ or a vector property of value $\overrightarrow{\boldsymbol{\Psi}}$, which is intrinsic to the model gas near the point source 102 at the time of a collision $t_{i}^{\prime}$.

In the point source 102 positioned in point $\overrightarrow{\boldsymbol{r}}^{\prime}$ at time $t_{i}^{\prime}$ and moving with velocity $\overrightarrow{\boldsymbol{u}}\left(t_{i}^{\prime}, \overrightarrow{\boldsymbol{r}}^{\prime}\right)$, a particle, as a properties carrier, obtains one or more of model gas properties being intrinsic to the model gas surrounding the point of the original collision at the time of the original collision. In the point source, the particle acquires a thermal velocity component $v_{\mathrm{T}}\left(t_{i}^{\prime}, \overrightarrow{\boldsymbol{r}}^{\prime}\right)$ of an arbitrary direction relatively to the point source. For certainty, the magnitude of the average thermal velocity component in three-dimensional configuration can be defined as:

$$
v_{\mathrm{T}}=\sqrt{\frac{3 k_{B} T}{m}}
$$

where $k_{B}$ is Boltzmann constant, $T$ is the temperature, and $m$ is the mass of a particle.

Each of the property carriers converging in a point sink 103 transfers in the point of the ending collision one or more of a set of properties, which may include just one property $\boldsymbol{\Psi}_{\boldsymbol{i n}}$, carried by each of the plurality of particles at the time of the ending collision.

\subsection{Principles of construction of the property balance in the model gas}

In the microscopic scale, the model gas flow is characterized by the group of particles of mass $m$, which move randomly and interact by collisions with effective collision cross-section $\sigma_{c}$. In each of a plurality of points in space at a given time, the particle density $n$, the magnitude of the random component of velocity or thermal velocity $v_{T}$, and the vector of mass flow velocity $\overrightarrow{\boldsymbol{u}}$ quantify the model gas. In the interests of simplicity, unless otherwise stated, the particles are considered to have a unit mass, which, in the presence of external force, are accelerated during ballistic traveling with acceleration $\overrightarrow{\boldsymbol{g}}$. We have recognized that each point in space occupied by the model gas may serve as both a sink or a collector of property delivered by 
converging ballistic particles from the entire model gas system and a source or a disperser into the surrounding of the property taken away by diverging ballistic particles.

Here, we reasonably may expect maintenance of a general property balance in each of a plurality of the points of collisions within the model gas system. We formulate the balance, illustrated as a word equation in Fig. 2 as follows. In a given non-moving point $\overrightarrow{\boldsymbol{r}}$ at given time $t$, the net rate of property influx per unit volume, $\boldsymbol{B}_{\boldsymbol{i n}}^{\Psi_{n} \mathrm{FS}}(\overrightarrow{\boldsymbol{r}}, t)$, formed the plurality of converging ballistic particles from the model gas system is equated to the temporal rate of property change per unit volume $\frac{\partial}{\partial t}[n \boldsymbol{\Psi}]$ and the net rate of property efflux per unit volume, $\boldsymbol{B}_{\text {out }}^{\boldsymbol{\Psi}_{\mathrm{F}} \mathrm{FS}}$, formed the plurality of diverging ballistic particles. This statement is expressed symbolically as

$$
\boldsymbol{B}_{\text {in }}^{\Psi_{-} \mathrm{FS}}(\overrightarrow{\boldsymbol{r}}, t)=\boldsymbol{B}_{\text {out }}^{\boldsymbol{\Psi}_{\mathrm{F}} \mathrm{FS}}(\overrightarrow{\boldsymbol{r}}, t)+\frac{\partial}{\partial t}[n(t, \overrightarrow{\boldsymbol{r}}) \boldsymbol{\Psi}(t, \overrightarrow{\boldsymbol{r}})] .
$$

For identification, we suggest calling the quantitative relationship above as the Ballistic Principle of the Property Balance in Space (BPPBS). The Principle applies in general to any gas system, including the model gas systems containing heterogeneous gas-solid interfaces. In addition, for clarity, we call our model as the Ballistic Model.

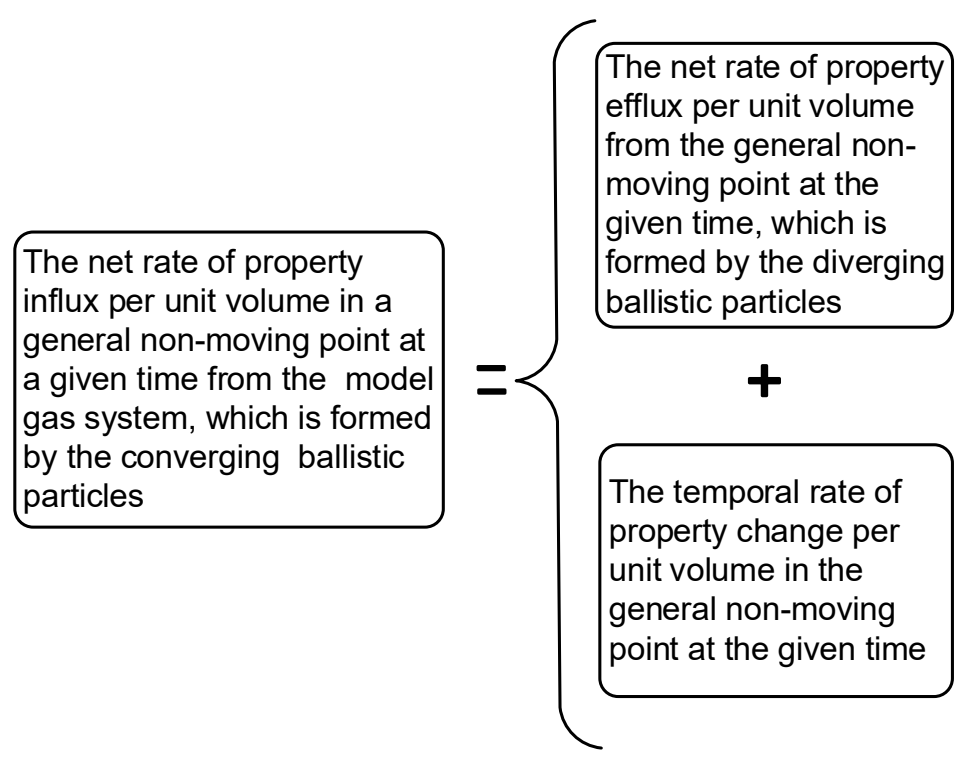

Fig. 2 Block diagram of a word equation of the general property balance in the model gas system

This conceptual relationship can be expanded to the infinite space, for example, in a hypothetical system with no gravitational force. Straight-line trajectories of the particles may start from the infinity.

Here, in the interests of simplicity, we concentrate our further analysis on the homogeneous model gas flow in the three-dimensional space having uniform gas properties on its periphery. Therefore, Equation (7) is reduced to:

$$
\boldsymbol{B}_{\text {in }}^{\Psi_{-} \mathrm{F}}(\overrightarrow{\boldsymbol{r}}, t)=\boldsymbol{B}_{\text {out }}^{\boldsymbol{\Psi}_{\mathrm{F}} \mathrm{FS}}(\overrightarrow{\boldsymbol{r}}, t)+\frac{\partial}{\partial t}[n(t, \overrightarrow{\boldsymbol{r}}) \boldsymbol{\Psi}(t, \overrightarrow{\boldsymbol{r}})],
$$

where $\boldsymbol{B}_{\boldsymbol{i n}}^{\boldsymbol{\Psi}_{\mathbf{n}} \mathrm{F}}$ is the net rate of property influx per unit volume, which is formed by the plurality of converging ballistic particles from the surrounding model gas in the given non- 
moving point $\overrightarrow{\boldsymbol{r}}$ at the given time $t$. Still, the space of the model gas system may be separated from the infinite space by defining, for example, non-uniform gas pressure over the surface confining the system. This situation is further discussed when analyzing the momentum balance.

\subsection{Defining a net rate of total property influx per unit volume in a general non-moving point at} a given time from the surrounding model gas

We have recognized that there exists a combination of a specific direction of an initial instant vector of thermal velocity $\overrightarrow{\boldsymbol{v}}_{\mathbf{T}}\left(t_{i}^{\prime}, \overrightarrow{\boldsymbol{r}}^{\prime}\right)$ and a vector of mass flow velocity $\overrightarrow{\boldsymbol{u}}\left(t_{i}^{\prime}, \overrightarrow{\boldsymbol{r}}^{\prime}\right)$, which provides an opportunity for each of the selected particles to arrive in the given non-moving point $\overrightarrow{\boldsymbol{r}}$ at the given time $t$. These particles, which are from the original collisions within the entire model gas system, form a converging flux in the given non-moving point of the space occupied by the model gas at the given time. In the following Table 1, various terms used in the equations and drawings are listed.

TABLE 1 . Terms and symbols used in the equations and drawings.

\begin{tabular}{|c|c|}
\hline Symbols & Short description \\
\hline$\nabla$ & $\boldsymbol{\nabla}=\overrightarrow{\boldsymbol{\imath}} \frac{\partial}{\partial x}+\overrightarrow{\boldsymbol{\jmath}} \frac{\partial}{\partial y}+\overrightarrow{\boldsymbol{k}} \frac{\partial}{\partial z}$ the operator of vector differentiation \\
\hline$t$ & given time \\
\hline$t_{i}^{\prime}$ & time of the original collision of the converging particle \\
\hline $\overrightarrow{\boldsymbol{r}}$ & the position of the ending point of the converging particle \\
\hline $\overrightarrow{\boldsymbol{r}}^{\prime}$ & is the position of the starting point of the converging particle \\
\hline $\overrightarrow{\boldsymbol{u}}\left(t_{i}^{\prime}, \overrightarrow{\boldsymbol{r}}^{\prime}\right)$ & $\begin{array}{l}\text { mass flow velocity or velocity of the point sink in the point } \overrightarrow{\boldsymbol{r}}^{\prime} \text { at time } \\
t_{i}^{\prime}\end{array}$ \\
\hline$v_{\mathrm{T}}\left(t_{i}^{\prime}, \overrightarrow{\boldsymbol{r}}^{\prime}\right)$ & $\begin{array}{l}\text { the average magnitude of the thermal velocity of the converging } \\
\text { particle in point } \overrightarrow{\boldsymbol{r}}^{\prime} \text { at time } t_{i}^{\prime}\end{array}$ \\
\hline$Z_{V}\left(t_{i}^{\prime}, \overrightarrow{\boldsymbol{r}}^{\prime}\right)$ & $\begin{array}{l}\text { the rate of collisions per unit volume in the point of the original } \\
\text { collision } \overrightarrow{\boldsymbol{r}}^{\prime} \text { at the time } t_{i}^{\prime} \text { of the original collision }\end{array}$ \\
\hline $\overrightarrow{\boldsymbol{v}}\left(t_{i}^{\prime}, \overrightarrow{\boldsymbol{r}}^{\prime}, t, \overrightarrow{\boldsymbol{r}}\right)$ & a velocity vector in the ending point $\overrightarrow{\boldsymbol{r}}$ at the given time $t$ \\
\hline$Q_{i}\left(t, t_{i}^{\prime}\right)$ & $\begin{array}{l}\text { the probability of traveling along the ballistic trajectory of the } \\
\text { converging ballistic trajectory starting at time } t_{i}^{\prime} \text { and ending at time } t\end{array}$ \\
\hline $\boldsymbol{\Psi}_{\text {in }}\left(t_{i}^{\prime}, \overrightarrow{\boldsymbol{r}}^{\prime}, t, \overrightarrow{\boldsymbol{r}}\right)$ & $\begin{array}{l}\text { a property content delivered by the converging ballistic particle in } \\
\text { the ending point } \overrightarrow{\boldsymbol{r}} \text { at the given time } t\end{array}$ \\
\hline$\varphi_{i}=t-t_{i}^{\prime}$ & $\begin{array}{l}\text { traveling time between an original and ending consecutive } \\
\text { collisions or the ballistic traveling time }\end{array}$ \\
\hline$n$ & particles density \\
\hline$m$ & particle mass \\
\hline$\sigma_{c}$ & the cross-section of collisions \\
\hline$P_{c}=\sigma_{c} n$ & the number of particles within a collision tube of a unit length \\
\hline
\end{tabular}




$$
\begin{aligned}
& \text { V } \\
& \overrightarrow{\boldsymbol{r}}_{\boldsymbol{i}}^{\boldsymbol{c}}\left(\overrightarrow{\boldsymbol{r}}^{\prime}, t_{i}^{\prime}, \tilde{t}\right) \\
& \overrightarrow{\boldsymbol{v}}_{\boldsymbol{i}}^{c}\left(\overrightarrow{\boldsymbol{r}}^{\prime}, t_{i}^{\prime}, \tilde{t}\right) \\
& \overrightarrow{\boldsymbol{n}}_{\boldsymbol{i}}= \\
& \overrightarrow{\boldsymbol{n}}_{\boldsymbol{i}}\left(t_{i}^{\prime}, \overrightarrow{\boldsymbol{r}}^{\prime}, t, \overrightarrow{\boldsymbol{r}}\right) \\
& =\frac{\overrightarrow{\boldsymbol{r}}-\overrightarrow{\boldsymbol{r}}_{\boldsymbol{i}}^{c}\left(\overrightarrow{\boldsymbol{r}}^{\prime}, t_{i}^{\prime}, t\right)}{\left|\overrightarrow{\boldsymbol{r}}-\overrightarrow{\boldsymbol{r}}_{\boldsymbol{i}}^{c}\left(\overrightarrow{\boldsymbol{r}}^{\prime}, t_{i}^{\prime}, t\right)\right|} \\
& \overrightarrow{\widetilde{\boldsymbol{r}}}=\overrightarrow{\boldsymbol{r}}\left(\overrightarrow{\boldsymbol{r}}^{\prime}, t_{i}^{\prime}, \tilde{t}\right) \\
& \overrightarrow{\widetilde{v}}=\overrightarrow{\boldsymbol{v}}\left(t_{i}^{\prime}, \overrightarrow{\boldsymbol{r}}^{\prime}, \tilde{t}\right) \\
& \overrightarrow{\mathbf{g}} \\
& v_{r e l}
\end{aligned}
$$

the cross-section of collisions

the volume of integration over space occupied by the model gas

the position of a virtual ballistic particle at a time $\tilde{t}$, which has zero magnitude of thermal velocity in the starting point $\overrightarrow{\boldsymbol{r}}^{\prime}$ at the time of the original collision $t_{i}^{\prime}$

the velocity vector of the virtual ballistic particle having a zero component of the thermal velocity at a time $\tilde{t}$

instant unit vector directing thermal velocity component, so a traveling particle targets point $\overrightarrow{\boldsymbol{r}}$ at time $t$

the position vector of a ballistic particle at time $\tilde{t}$

the velocity vector of the ballistic particle at time $\tilde{t}$

the external force applied to a particle of a unit mass

the average magnitude of the relative velocity or the velocity of the traveling particle with respect to a nearby passed particle

Fig. 3 shows a three-dimensional view of the model gas system for explaining a ballistic movement of the particle after a collision, which is affected by the external field of force. For clarity, the observer's Cartesian coordinate system 100 is oriented, so y-axis is along the negative direction of the applied acceleration field 306, which provides, for each particle, acceleration $\overrightarrow{\boldsymbol{g}}$.

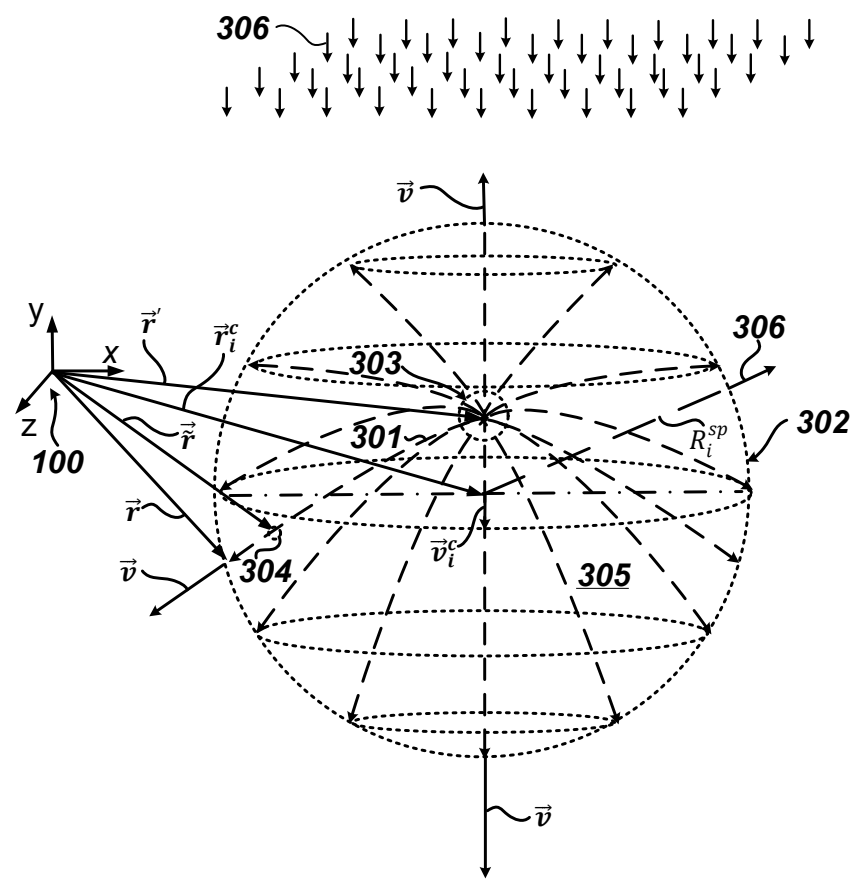

Fig. 3 Schematic three-dimensional view of the model gas system for explaining the ballistic movement of the particle after a collision 
We define the net rate of property influx from the model gas in the general non-moving point $\overrightarrow{\boldsymbol{r}}$ at the given time $t$ by these six steps:

Step (1) Identifying the converging ballistic trajectory and trajectory characteristics

Step (1) includes:

(1) Formulating position vector $\overrightarrow{\tilde{\boldsymbol{r}}}=\overrightarrow{\boldsymbol{r}}\left(\overrightarrow{\boldsymbol{r}}^{\prime}, t_{i}^{\prime}, \tilde{t}\right)$ of particle 304 on trajectory 301 and velocity vector $\overrightarrow{\widetilde{v}}=\overrightarrow{\boldsymbol{v}}(\tilde{t})$ (not shown) at time $\tilde{t}$ by applying Equations (9) and (10), respectively, given below:

$$
\overrightarrow{\tilde{\boldsymbol{r}}}=\overrightarrow{\boldsymbol{r}}\left(\overrightarrow{\boldsymbol{r}}^{\prime}, t_{i}^{\prime}, \tilde{t}\right)=v_{\mathrm{T}}\left(t_{i}^{\prime}, \overrightarrow{\boldsymbol{r}}^{\prime}\right)\left(\tilde{t}-t_{i}^{\prime}\right) \overrightarrow{\boldsymbol{n}}_{\boldsymbol{i}}+\overrightarrow{\boldsymbol{r}}_{\boldsymbol{i}}^{c}\left(\overrightarrow{\boldsymbol{r}}^{\prime}, t_{i}^{\prime}, \tilde{t}\right)
$$

and

$$
\overrightarrow{\widetilde{\boldsymbol{v}}}=\overrightarrow{\boldsymbol{v}}\left(\overrightarrow{\boldsymbol{r}}^{\prime}, t_{i}^{\prime}, \tilde{t}\right)=v_{T}\left(t_{i}^{\prime}, \overrightarrow{\boldsymbol{r}}^{\prime}\right) \overrightarrow{\boldsymbol{n}}_{\boldsymbol{i}}+\overrightarrow{\boldsymbol{v}}_{\boldsymbol{i}}^{c}\left(\overrightarrow{\boldsymbol{r}}^{\prime}, t_{i}^{\prime}, \tilde{t}\right),
$$

where $t \geq \tilde{t} \geq t_{i}^{\prime}, \overrightarrow{\boldsymbol{n}}_{\boldsymbol{i}}$ is defined in Table 1, and $t_{i}^{\prime}$ is defined in (2) below. In Equations (9) and (10) above, $\overrightarrow{\boldsymbol{r}}_{\boldsymbol{i}}^{\boldsymbol{c}}\left(\overrightarrow{\boldsymbol{r}}^{\prime}, t_{i}^{\prime}, \tilde{t}\right)$ and $\overrightarrow{\boldsymbol{v}}_{\boldsymbol{i}}^{\boldsymbol{c}}\left(\overrightarrow{\boldsymbol{r}}^{\prime}, t_{i}^{\prime}, \tilde{t}\right)$ are defined by an appropriate law of motion.

Specifically, when Newton's Laws of Motion govern the motion of particles, then $\overrightarrow{\boldsymbol{r}}_{\boldsymbol{i}}^{\boldsymbol{c}}\left(\overrightarrow{\boldsymbol{r}}^{\prime}, t_{i}^{\prime}, \tilde{t}\right)$ and $\overrightarrow{\boldsymbol{v}}_{\boldsymbol{i}}^{c}\left(\overrightarrow{\boldsymbol{r}}^{\prime}, t_{i}^{\prime}, \tilde{t}\right)$ are defined by Equations (11) and (12) given below:

$$
\overrightarrow{\boldsymbol{r}}_{i}^{c}\left(\overrightarrow{\boldsymbol{r}}^{\prime}, t_{i}^{\prime}, \tilde{t}\right)=\overrightarrow{\boldsymbol{r}}^{\prime}+\overrightarrow{\boldsymbol{u}}\left(t_{i}^{\prime}, \overrightarrow{\boldsymbol{r}}^{\prime}\right)\left(\tilde{t}-t_{i}^{\prime}\right)+\frac{1}{2} \overrightarrow{\mathbf{g}}\left(\tilde{t}-t_{i}^{\prime}\right)^{2}
$$

and

$$
\overrightarrow{\boldsymbol{v}}_{\boldsymbol{i}}^{c}\left(\overrightarrow{\boldsymbol{r}}^{\prime}, t_{i}^{\prime}, \tilde{t}\right)=\overrightarrow{\boldsymbol{u}}\left(t_{i}^{\prime}, \overrightarrow{\boldsymbol{r}}^{\prime}\right)+\overrightarrow{\mathbf{g}}\left(\tilde{t}-t_{i}^{\prime}\right) .
$$

(2) Determining the time of the original collision $t_{i}^{\prime}$ in point $\overrightarrow{\boldsymbol{r}}^{\prime}$. It can be done by solving Equation (9) in which $\tilde{t}=t$. Where a model gas system is governed by Newton's Law of Motion, it can be done by resolving, for each of the plurality of ballistic particles, the equation of projectile motion, given by Equation (13), with respect to the ballistic traveling time, $\varphi_{i}$ :

$$
\frac{1}{2} \overrightarrow{\mathbf{g}} \varphi_{i}^{2}+\left[v_{\mathrm{T}}\left(t_{i}^{\prime}, \overrightarrow{\boldsymbol{r}}^{\prime}\right) \overrightarrow{\boldsymbol{n}}_{\boldsymbol{i}}+\overrightarrow{\boldsymbol{u}}\left(t_{i}^{\prime}, \overrightarrow{\boldsymbol{r}}^{\prime}\right)\right] \varphi_{i}+\overrightarrow{\boldsymbol{r}}^{\prime}-\overrightarrow{\boldsymbol{r}}=0,
$$

which is obtained by substitution of Equation (11) in Equation (9) followed by the assignment of $\tilde{t}=t$ and substitution of $\varphi_{i}$ defined in Table 1 in Equation (9) and rearrangement of the terms. Upon resolving Equation (13) and selecting meaningful values for $\varphi_{i}, t_{i}^{\prime}$ is computed as follows

$$
t_{i}^{\prime}=t-\varphi_{i}
$$

for each of the plurality of ballistic particles.

(3) Defining an appropriate instant unit vector directing thermal velocity component of each particle in point $\overrightarrow{\boldsymbol{r}}^{\prime}$ at time $t_{i}^{\prime}$ by presenting Equation (10) in the following form:

$$
\varphi_{i} v_{\mathrm{T}} \overrightarrow{\boldsymbol{n}}_{\boldsymbol{i}}=\overrightarrow{\boldsymbol{r}}-\overrightarrow{\boldsymbol{r}}_{\boldsymbol{i}}^{\boldsymbol{c}},
$$

where $\overrightarrow{\boldsymbol{r}}_{\boldsymbol{i}}^{c}$ is defined as:

$$
\overrightarrow{\boldsymbol{r}}_{\boldsymbol{i}}^{\boldsymbol{c}}\left(\overrightarrow{\boldsymbol{r}}^{\prime}, t_{i}^{\prime}, t\right)=\overrightarrow{\boldsymbol{r}}^{\prime}+\overrightarrow{\boldsymbol{u}}\left(t_{i}^{\prime}, \overrightarrow{\boldsymbol{r}}^{\prime}\right) \varphi_{i}+\frac{1}{2} \overrightarrow{\mathbf{g}}\left(\varphi_{i}\right)^{2} .
$$


In this, vector $\overrightarrow{\boldsymbol{r}}_{\boldsymbol{i}}^{c}$ is interpreted as the position of the center of the expansion zone at time $t$ or the position of a particle having zero magnitude of an arbitrary or thermal velocity in point $\overrightarrow{\boldsymbol{r}}^{\prime}$ at the time $t_{i}^{\prime}$ of the divergence, which is observed at time $t$.

(4) Defining the size of the expansion zone $R_{i}^{s p}$, by executing scalar multiplication of Equation (15) on itself resulted and averaging as:

$$
\varphi_{i}^{2} v_{T}^{2}=\left(\overrightarrow{\boldsymbol{r}}-\overrightarrow{\boldsymbol{r}}_{\boldsymbol{i}}^{c}\right)^{\mathbf{2}}
$$

and computing $R_{i}^{s p}$ from Equation (17) as

$$
R_{i}^{s p}=\varphi_{i} v_{\mathrm{T}}=\left|\overrightarrow{\boldsymbol{r}}-\overrightarrow{\boldsymbol{r}}_{\boldsymbol{i}}^{c}\right| .
$$

The velocity vector $\overrightarrow{\boldsymbol{v}}_{\boldsymbol{i}}^{\boldsymbol{c}}$ of the center of the expansion zone 302 at time $t$ is computed as

$$
\overrightarrow{\boldsymbol{v}}_{\boldsymbol{i}}^{c}\left(\overrightarrow{\boldsymbol{r}}^{\prime}, t_{i}^{\prime}, t\right)=\overrightarrow{\boldsymbol{u}}\left(t_{i}^{\prime}, \overrightarrow{\boldsymbol{r}}^{\prime}\right)+\overrightarrow{\boldsymbol{g}} \varphi_{i}
$$

The velocity $\overrightarrow{\boldsymbol{v}}$ of each particle reaching the general non-moving point $\overrightarrow{\boldsymbol{r}}$ at time $t$ on any point of the control surface 302 is computed as

$$
\overrightarrow{\boldsymbol{v}}\left(\overrightarrow{\boldsymbol{r}}^{\prime}, t_{i}^{\prime}, t\right)=v_{T}\left(t_{i}^{\prime}, \overrightarrow{\boldsymbol{r}}^{\prime}\right) \overrightarrow{\boldsymbol{n}}_{\boldsymbol{i}}+\overrightarrow{\boldsymbol{u}}\left(t_{i}^{\prime}, \overrightarrow{\boldsymbol{r}}^{\prime}\right)+\overrightarrow{\mathbf{g}} \varphi_{i},
$$

which is obtained by assigning $\tilde{t}=t$ and substitution of $\varphi_{i}=t-t_{i}^{\prime}$ in Equation (10) given above and rearrangement of the terms.

Step (2) Defining the probability of free path traveling along the ballistic trajectory from the starting point to the ending point

Step (2) includes:

(1) Defining the average magnitude of the velocity of the traveling particle with respect to nearby passed particles at a particular point of a trajectory.

(2) Representing the probability of traveling along the ballistic trajectory from the starting point to the ending point in the three-dimensional configuration by Equation (21) given below:

$$
Q_{i}\left(t, t_{i}^{\prime}\right)=\exp \left(-\int_{t_{i}^{\prime}}^{t} P_{c}(\overrightarrow{\tilde{r}}(\tilde{t})) v_{r e l}(\overrightarrow{\tilde{r}}(\tilde{t})) \mathrm{d} \tilde{t}\right),
$$

where $\tilde{t}$ is a parametric time $t_{i}^{\prime}<\tilde{t} \leq t, \overrightarrow{\widetilde{r}}(\tilde{t})$ is a trajectory point of the ballistic trajectory at the parametric time $\tilde{t}, v_{\text {rel }}(\overrightarrow{\tilde{\boldsymbol{r}}}(\tilde{t}))$ is an average magnitude of the relative velocity with respect to a nearby passed particle in the trajectory point $\overrightarrow{\overrightarrow{\boldsymbol{r}}}(\tilde{t})$, and $P_{c}(\overrightarrow{\widetilde{\boldsymbol{r}}}(\tilde{t}))$ is the average number of collisions per unit length in the trajectory point $\overrightarrow{\widetilde{r}}(\tilde{t})$.

Fig. 4 shows a schematic view for explaining a method for determining the average magnitude of the instant velocity of the ballistic particle with respect to a nearby passed particle along the ballistic trajectory of the traveling particle in a three-dimensional configuration. For clarity, the observer's Cartesian coordinate system 100 is oriented, so y-axis is along the negative direction of the applied acceleration field, 401, which provides, for each particle, acceleration $\overrightarrow{\boldsymbol{g}}$. Here, at time $\tilde{t}$, particle P1 having velocity $\overrightarrow{\boldsymbol{v}}_{\mathbf{1}}\left(t_{i}^{\prime}, \overrightarrow{\boldsymbol{r}}^{\prime}, \tilde{t}\right)$ and originated from a collision in point $\mathrm{A}$, passes in point $\mathrm{B}$, which is positioned on ballistic trajectory 402 , particle P2 having velocity $\overrightarrow{\boldsymbol{v}}_{\mathbf{2}}(\overrightarrow{\widetilde{\boldsymbol{r}}}(\tilde{t}))$. 


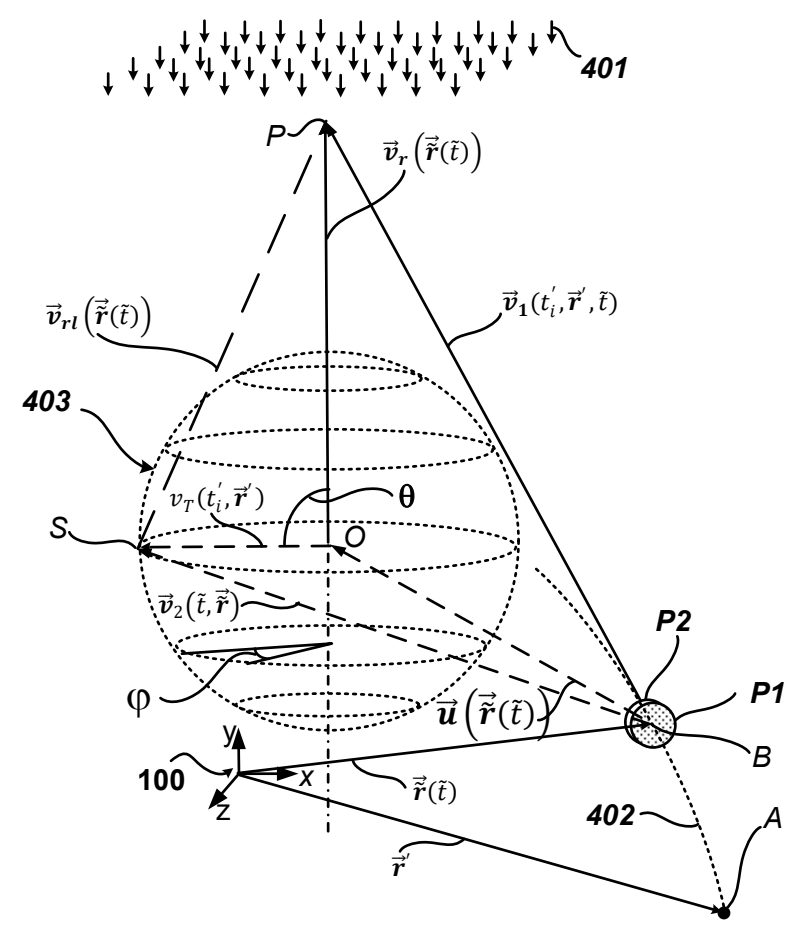

Fig. 4 Schematic view for explaining a method for determining the average magnitude of the instant velocity of the traveling particle with respect to a nearby passed particle along a ballistic trajectory of the traveling particle in a three-dimensional configuration

Following sub-steps calculate the average magnitude of the velocity $v_{r e l}(\overrightarrow{\widetilde{r}}(\tilde{t}))$ of the traveling particle with respect to nearby passed particles at a particular point of a trajectory $\mathrm{B}$ at a specified time $\tilde{t}$ :

(1) by defining an instant magnitude of the velocity of one of the plurality of converging ballistic particles in the trajectory point with respect to one of a plurality of nearby particles in the trajectory point $\overrightarrow{\widetilde{\boldsymbol{r}}}$ at time $\tilde{t}$ as

$$
\overrightarrow{\boldsymbol{v}}_{\boldsymbol{r l}}(\overrightarrow{\widetilde{\boldsymbol{r}}}(\tilde{t}))=\overrightarrow{\boldsymbol{v}}_{\mathbf{1}}\left(t_{i}^{\prime}, \overrightarrow{\boldsymbol{r}}^{\prime}, \tilde{t}\right)-\overrightarrow{\boldsymbol{v}}_{\mathbf{2}}(\overrightarrow{\widetilde{\boldsymbol{r}}}(\tilde{t}))
$$

which is formed by connecting the end of the instant velocity vector $\overrightarrow{\boldsymbol{v}}_{2}(\tilde{t}, \overrightarrow{\tilde{\boldsymbol{r}}})$ with any point S on spherical surface 403 of radius $v_{T}(\overrightarrow{\widetilde{r}}(\tilde{t}))$, where

$$
\overrightarrow{\boldsymbol{v}}_{\mathbf{1}}\left(t_{i}^{\prime}, \overrightarrow{\boldsymbol{r}}^{\prime}, \tilde{t}\right)=v_{T}\left(t_{i}^{\prime}, \overrightarrow{\boldsymbol{r}}^{\prime}\right) \overrightarrow{\boldsymbol{n}}_{\mathbf{1}}+\overrightarrow{\boldsymbol{u}}\left(t_{i}^{\prime}, \overrightarrow{\boldsymbol{r}}^{\prime}\right)+\overrightarrow{\mathbf{g}}\left(\tilde{t}-t_{i}^{\prime}\right),
$$

where $\overrightarrow{\boldsymbol{n}}_{\mathbf{1}}$ is a unit vector of a specific direction having the point of origin $\overrightarrow{\boldsymbol{r}}^{\prime}, v_{T}\left(t_{i}^{\prime}, \overrightarrow{\boldsymbol{r}}^{\prime}\right)$ and $\overrightarrow{\boldsymbol{u}}\left(t_{i}^{\prime}, \overrightarrow{\boldsymbol{r}}^{\prime}\right)$ are thermal velocity and mass flow velocity components in the rest frame of the model gas in point $\overrightarrow{\boldsymbol{r}}^{\prime}$ at time $t_{i}^{\prime}$, which are acquired by particle P1 because of a collision in this point, and where

$$
\overrightarrow{\boldsymbol{v}}_{\mathbf{2}}(\overrightarrow{\widetilde{\boldsymbol{r}}}(\tilde{t}))=v_{T}(\overrightarrow{\widetilde{\boldsymbol{r}}}(\tilde{t})) \overrightarrow{\boldsymbol{n}}_{\boldsymbol{i}}+\overrightarrow{\boldsymbol{u}}(\overrightarrow{\widetilde{\boldsymbol{r}}}(\tilde{t})),
$$

where $v_{T}(\overrightarrow{\widetilde{\boldsymbol{r}}}(\tilde{t}))$ and $\overrightarrow{\boldsymbol{u}}(\overrightarrow{\tilde{\boldsymbol{r}}}(\tilde{t}))$ are thermal velocity and mass flow velocity components in the rest frame of the model gas in point $\overrightarrow{\widetilde{r}}$ at time $\tilde{t}$, which are acquired by particle P2 because of a collision in this point and 
(2) by averaging the instant magnitude of relative velocity over a plurality of directions of a thermal velocity component of one of the nearby particles in the trajectory point $\overrightarrow{\widetilde{r}}$ at time $\tilde{t}$. This is done by integrating the instant magnitude of the relative velocity of Equation (24) over angles of $\vartheta$ from 0 to $\pi$ and $\varphi$, which is the angle of rotation around axis OP from 0 to $2 \pi$ followed by normalizing by the solid angle of $4 \pi$, which results in:

$$
\begin{aligned}
& v_{\text {rel }}(\overrightarrow{\widetilde{r}}(\tilde{t}))= \\
& \frac{1}{2} \int_{0}^{\pi} \sqrt{\left|\overrightarrow{\boldsymbol{v}}_{\boldsymbol{r}}(\overrightarrow{\overrightarrow{\boldsymbol{r}}}(\tilde{t}))\right|^{2}+\left[v_{T}(\overrightarrow{\overrightarrow{\boldsymbol{r}}}(\tilde{t}))\right]^{2}-2 v_{T}(\overrightarrow{\overrightarrow{\boldsymbol{r}}}(\tilde{t}))\left|\overrightarrow{\boldsymbol{v}}_{\boldsymbol{r}}(\overrightarrow{\overrightarrow{\boldsymbol{r}}}(\tilde{t}))\right| \cos (\vartheta)} \sin (\vartheta) \mathrm{d} \vartheta,
\end{aligned}
$$

where

$$
\overrightarrow{\boldsymbol{v}}_{\boldsymbol{r}}(\overrightarrow{\tilde{\boldsymbol{r}}}(\tilde{t}))=\overrightarrow{\boldsymbol{v}}_{\mathbf{1}}\left(t_{i}^{\prime}, \overrightarrow{\boldsymbol{r}}^{\prime}, \tilde{t}\right)-\overrightarrow{\boldsymbol{u}}(\overrightarrow{\tilde{\boldsymbol{r}}}(\tilde{t})) .
$$

Note that, usually, the magnitude of the relative mass flow velocity or the mass flow velocity component of the passing particle $\mathrm{P} 1$ with respect to nearby passed particle $\mathrm{P} 2$ is insignificant in comparison with the magnitude of the thermal velocity of either passing particle P1 or nearby passed particle $\mathrm{P} 2$ or of both.

The average magnitude of the relative velocity of the traveling particle in the trajectory point is calculated from Equation (27) given below, which is obtained by substitution of $\left|\overrightarrow{\boldsymbol{v}}_{\boldsymbol{r}}\right| \cong$ $v_{T}\left(t_{i}^{\prime}, \overrightarrow{\boldsymbol{r}}^{\prime}\right)$ in Equation (25) and executing the integration of the resulted equation:

$$
v_{r e l}(\overrightarrow{\tilde{\boldsymbol{r}}}(\tilde{t}))=\frac{1}{6 v_{T}\left(t_{i}^{\prime}, \overrightarrow{\boldsymbol{r}}^{\prime}\right) v_{T}(\tilde{t}, \overrightarrow{\overrightarrow{\boldsymbol{r}}})}\left\{\left(v_{T}\left(t_{i}^{\prime}, \overrightarrow{\boldsymbol{r}}^{\prime}\right)+v_{T}(\overrightarrow{\tilde{\boldsymbol{r}}}(\tilde{t}))\right)^{3}-\left|v_{T}\left(t_{i}^{\prime}, \overrightarrow{\boldsymbol{r}}^{\prime}\right)-v_{T}(\overrightarrow{\tilde{\boldsymbol{r}}}(\tilde{t}))\right|^{3}\right\}
$$

where $v_{\text {rel }}(\overrightarrow{\tilde{\boldsymbol{r}}}(\tilde{t}))$ is the average magnitude of relative velocity in the trajectory point, $v_{T}\left(t_{i}^{\prime}, \overrightarrow{\boldsymbol{r}}^{\prime}\right)$ is the average magnitude of the thermal velocity component in the starting point of the ballistic trajectory, and $v_{T}(\overrightarrow{\widetilde{r}}(\tilde{t}))$ is the average magnitude of the thermal velocity of a nearby passed particle in the trajectory point.

In addition, usually, the magnitudes of the thermal velocity of nearby particles are approximately identical. For non-relativistic particles, the average magnitude of the relative velocity with respect to each particle moving in an arbitrary direction is calculated from Equation (28) given below, which is obtained by substitution of $v_{T}(\overrightarrow{\overrightarrow{\boldsymbol{r}}}(\tilde{t}))=v_{T}\left(t_{i}^{\prime}, \overrightarrow{\boldsymbol{r}}^{\prime}\right)=v_{T}$ in Equation (27):

$$
v_{\text {rel }}=\frac{4}{3} v_{T}
$$

Analogously, for relativistic particles, the average magnitude of the relative velocity with respect to each particle moving in an arbitrary direction is calculated from Equation (28) given below:

$$
v_{r e l}=\frac{v_{T}}{2} \int_{0}^{\pi} \frac{\sqrt{2-2 \cos (\vartheta)-\left[1-\cos ^{2}(\vartheta)\right] v_{T}^{2} / c^{2}}}{1-v_{T}^{2} \cos (\vartheta) / c^{2}} \sin (\vartheta) \mathrm{d} \vartheta,
$$

where $c$ is the speed of light. For $\frac{v_{T}}{c} \ll 1$, integrating Equation (29) will yield Equation (28). For $\frac{v_{T}}{c} \cong 1$, integrating Equation (29) will yield: 


$$
v_{\text {rel }} \cong c \text {. }
$$

Step (3) Defining the net rate of particle efflux per unit volume from a point source positioned in a point of the original collisions and moving with the mass flow velocity of the model gas in that point

Step (3) includes the following sub-steps:

(1) Defining the vector field of the particle flux $\vec{J}_{\boldsymbol{r}^{\prime}}^{\mathrm{N}}$ along each of the ballistic trajectories in a point of the space $\overrightarrow{\boldsymbol{r}}$ around the point of the original collision, the step that includes representing $\overrightarrow{\boldsymbol{J}}_{\boldsymbol{r}^{\prime}}^{\mathrm{N}}$ by applying Equation (31) as given below:

$$
\overrightarrow{\boldsymbol{J}}_{\boldsymbol{r}^{\prime}}^{\mathbf{N}}=\frac{1}{2} n\left(t_{i}^{\prime}, \overrightarrow{\boldsymbol{r}}^{\prime}\right) Q_{i}\left(t, t_{i}^{\prime}\right) \overrightarrow{\boldsymbol{v}}\left(t_{i}^{\prime}, \overrightarrow{\boldsymbol{r}}^{\prime}, t, \overrightarrow{\boldsymbol{r}}\right),
$$

where $\overrightarrow{\boldsymbol{v}}$ is defined by Equation (20), $Q_{i}$ is a survival probability defined by Equation (21), and $n\left(t_{i}^{\prime}, \overrightarrow{\boldsymbol{r}}^{\prime}\right)$ is particle density at a specific point $\overrightarrow{\boldsymbol{r}}^{\prime}$ at time $t_{i}^{\prime}$.

(2) Representing, in a coordinate system associated with point $\overrightarrow{\boldsymbol{r}}_{\boldsymbol{i}}^{\boldsymbol{c}}$ moving with velocity $\overrightarrow{\boldsymbol{v}}_{\boldsymbol{i}}^{\boldsymbol{c}}$, the vector field of the particle vector flux $\vec{J}_{\boldsymbol{r}^{\prime}}^{\mathbf{N}}$ through the in the control surface 302 of Fig. 3 in the following form:

$$
\overrightarrow{\boldsymbol{J}}_{C S}^{\mathrm{N}}=\frac{1}{2} n\left(t_{i}^{\prime}, \overrightarrow{\boldsymbol{r}}^{\prime}\right) Q_{i}\left(t, t_{i}^{\prime}\right)\left[\overrightarrow{\boldsymbol{v}}\left(t_{i}^{\prime}, \overrightarrow{\boldsymbol{r}}^{\prime}, t, \overrightarrow{\boldsymbol{r}}\right)-\overrightarrow{\boldsymbol{v}}_{\boldsymbol{i}}^{c}\right]=\frac{1}{2} n\left(t_{i}^{\prime}, \overrightarrow{\boldsymbol{r}}^{\prime}\right) Q_{i}\left(t, t_{i}^{\prime}\right) v_{T}\left(t_{i}^{\prime}, \overrightarrow{\boldsymbol{r}}^{\prime}\right) \overrightarrow{\boldsymbol{n}}_{\boldsymbol{i}}
$$

(3) Applying and executing the divergence operator $\boldsymbol{\nabla}^{\prime} \cdot$ to the vector field of Equation (32) followed by shrinking the volume of the auxiliary control volume to infinitely small volume, i.e., $\overrightarrow{\boldsymbol{r}} \rightarrow \overrightarrow{\boldsymbol{r}}^{\prime}$, which, in formula form, is expressed as:

$$
Z_{V}\left(t_{i}^{\prime}, \overrightarrow{\boldsymbol{r}}^{\prime}\right)=\left\{\boldsymbol{\nabla}^{\prime} \cdot\left[\vec{J}_{C S}^{\mathrm{N}}\right]\right\}_{\overrightarrow{\boldsymbol{r}} \rightarrow \overrightarrow{\boldsymbol{r}}^{\prime}}=\frac{1}{2}\left\{\boldsymbol{\nabla}^{\prime} \cdot\left[n\left(t_{i}^{\prime}, \overrightarrow{\boldsymbol{r}}^{\prime}\right) Q_{i}\left(t, t_{i}^{\prime}\right) v_{T}\left(t_{i}^{\prime}, \overrightarrow{\boldsymbol{r}}^{\prime}\right) \overrightarrow{\boldsymbol{n}}_{\boldsymbol{i}}\right]\right\}_{\overrightarrow{\boldsymbol{r}} \rightarrow \overrightarrow{\boldsymbol{r}}^{\prime}},
$$

where

$$
\nabla^{\prime}=\overrightarrow{\boldsymbol{\imath}} \frac{\partial}{\partial x^{\prime}}+\overrightarrow{\boldsymbol{J}} \frac{\partial}{\partial y^{\prime}}+\overrightarrow{\boldsymbol{k}} \frac{\partial}{\partial z^{\prime}},
$$

and includes representing the particle flux production rate, or the net rate of particle efflux per unit volume, or the rate of collisions per unit volume $Z_{V}\left(t_{i}^{\prime}, \overrightarrow{\boldsymbol{r}}^{\prime}\right)$, in a point of the original collision moving with the mass flow velocity $\boldsymbol{u}\left(t_{i}^{\prime}, \overrightarrow{\boldsymbol{r}}^{\prime}\right)$ at timet $t_{i}^{\prime}$ by following Equation (35) given below:

$$
Z_{V}\left(t_{i}^{\prime}, \overrightarrow{\boldsymbol{r}}^{\prime}\right)=\frac{1}{2} n\left(t_{i}^{\prime}, \overrightarrow{\boldsymbol{r}}^{\prime}\right) P_{c}\left(t_{i}^{\prime}, \overrightarrow{\boldsymbol{r}}^{\prime}\right) \boldsymbol{v}_{\boldsymbol{r e l}}\left(t_{i}^{\prime}, \overrightarrow{\boldsymbol{r}}^{\prime}\right),
$$

where $n\left(t_{i}^{\prime}, \overrightarrow{\boldsymbol{r}}^{\prime}\right)$ is particle density in the corresponding point of original collisions at the time of the original collision, $P_{c}\left(t_{i}^{\prime}, \overrightarrow{\boldsymbol{r}}^{\prime}\right)$ is the average number of collisions per unit length in the corresponding point of original collisions at the time of the original collision, and $\boldsymbol{v}_{\text {rel }}\left(t_{i}^{\prime}, \overrightarrow{\boldsymbol{r}}^{\prime}\right)$ is the average magnitude of the relative velocity in the corresponding point of original collisions at the time of the original collision. The above is obtained upon recognizing that control volume 305 , which is confined by inflated control surface 302, is isolated (see Fig. 3), and then the total particle efflux through the surface 302 at time $t$ should be equal to the total particle efflux from the point at $\overrightarrow{\boldsymbol{r}}^{\prime}$ at time $t_{i}^{\prime}$ through the surface 303 closely surrounding point $\overrightarrow{\boldsymbol{r}}^{\prime}$. Some also 


\section{(c) $)(1) \Theta(\Theta$}

recognize that, after a collision in point $\overrightarrow{\boldsymbol{r}}^{\prime}$ at time $t_{i}^{\prime}$, the particle can move in any direction because of the arbitrary nature of the unit vector $\overrightarrow{\boldsymbol{n}}_{\boldsymbol{i}}$, thus forming an expansion zone around the point source in point $\overrightarrow{\boldsymbol{r}}^{\prime}$ at time $t_{i}^{\prime}$, which is shown as 302 .

Step (4) Defining property flux in a given non-moving point at a given time from one of a plurality of point sources of the model gas

Step (4) includes representing the property vector flux $\overrightarrow{\boldsymbol{J}}_{\overrightarrow{\boldsymbol{r}}^{\prime} \rightarrow \overrightarrow{\boldsymbol{r}}}^{\Psi}$ originated from the point source of the original collisions in point $\overrightarrow{\boldsymbol{r}}^{\prime}$ at time $t_{i}^{\prime}$, which moves in the space of the model gas with a mass-flow velocity $\overrightarrow{\boldsymbol{u}}\left(t_{i}^{\prime}, \overrightarrow{\boldsymbol{r}}^{\prime}\right)$, and being sensed by a point sink positioned in a being at rest point $\overrightarrow{\boldsymbol{r}}$ at the given time $t$ :

$$
\overrightarrow{\boldsymbol{J}}_{\overrightarrow{\boldsymbol{r}}^{\prime} \rightarrow \overrightarrow{\boldsymbol{r}}}^{\Psi}(t, \overrightarrow{\boldsymbol{r}})=\frac{1}{4 \pi} \frac{1}{\left|\overrightarrow{\boldsymbol{r}}-\overrightarrow{\boldsymbol{r}}_{\boldsymbol{i}}^{c}\right|^{2}} Q_{i}\left(t, t_{i}^{\prime}\right) Z_{V}\left(t_{i}^{\prime}, \overrightarrow{\boldsymbol{r}}^{\prime}\right) \frac{\overrightarrow{\boldsymbol{v}}\left(t_{i}^{\prime}, \overrightarrow{\boldsymbol{r}}^{\prime}, t, \overrightarrow{\boldsymbol{r}}\right)}{v_{T}\left(t_{i}^{\prime}, \overrightarrow{\boldsymbol{r}}^{\prime}\right)} \Psi_{\boldsymbol{i n}}\left(t_{i}^{\prime}, \overrightarrow{\boldsymbol{r}}^{\prime}, t, \overrightarrow{\boldsymbol{r}}\right) d V^{\prime}
$$

Step (5) Defining the rate of the property vector flux $\overrightarrow{\boldsymbol{J}}_{\boldsymbol{F} \boldsymbol{S} \rightarrow \overrightarrow{\boldsymbol{r}}}^{\Psi}$ in point $\overrightarrow{\boldsymbol{r}}$ at the given time $t$, which is originated from original collisions within entire space occupied by the model gas

Step (5) includes applying Equation (37) given below, which is obtained by integrating Equation (36) over the volume of the model gas system:

$$
\overrightarrow{\boldsymbol{J}}_{\boldsymbol{F} S \rightarrow \overrightarrow{\boldsymbol{r}}}^{\Psi}=\iiint_{\mathrm{V}} \frac{1}{4 \pi} \frac{1}{\left|\overrightarrow{\boldsymbol{r}}-\overrightarrow{\boldsymbol{r}}_{i}^{c}\right|^{2}} Q_{i}\left(t, t_{i}^{\prime}\right) Z_{V}\left(t_{i}^{\prime}, \overrightarrow{\boldsymbol{r}}^{\prime}\right) \frac{\overrightarrow{\boldsymbol{v}}\left(t_{i}^{\prime}, \overrightarrow{\boldsymbol{r}}^{\prime}, t, \overrightarrow{\boldsymbol{r}}^{\prime}\right)}{v_{T}\left(t_{i}^{\prime}, \overrightarrow{\boldsymbol{r}}^{\prime}\right)} \Psi_{\boldsymbol{i n}}\left(t_{i}^{\prime}, \overrightarrow{\boldsymbol{r}}^{\prime}, t, \overrightarrow{\boldsymbol{r}}\right) d V^{\prime} .
$$

Here point $\overrightarrow{\boldsymbol{r}}$ is excluded from integration in the equation above because we are interested in calculating the total rate of the property flux in the point sink at $\vec{r}$, which is originated from the surrounding point sources of the original collisions at $\overrightarrow{\boldsymbol{r}}^{\prime}$.

Step (6) Defining the net rate of property influx per unit volume $\boldsymbol{B}_{\text {in }}^{{ }_{-} F S}$ formed by the flow of ballistic particles carrying property and converging from the space occupied by the model gas in the general non-moving point of the space occupied by the model gas at the given time

Step (6) further includes a step of representing $\boldsymbol{B}_{\boldsymbol{i n}}^{\Psi-F S}$ by applying Equation (38) given below:

$$
\boldsymbol{B}_{\boldsymbol{i n}}^{\Psi-\mathrm{FS}}(\overrightarrow{\boldsymbol{r}}, t)=-\boldsymbol{\nabla} \cdot \iiint_{\mathrm{V}} \frac{1}{4 \pi} \frac{1}{\left|\overrightarrow{\boldsymbol{r}}-\overrightarrow{\boldsymbol{r}}_{\boldsymbol{i}}^{c}\right|^{2}} Q_{i}\left(t, t_{i}^{\prime}\right) Z_{V}\left(t_{i}^{\prime}, \overrightarrow{\boldsymbol{r}}^{\prime}\right) \frac{\overrightarrow{\boldsymbol{v}}^{\prime}\left(t_{i}^{\prime} \overrightarrow{\boldsymbol{r}}^{\prime}, t, \overrightarrow{\boldsymbol{r}}^{\prime}\right)}{v_{T}\left(t_{i}^{\prime}, \overrightarrow{\boldsymbol{r}}^{\prime}\right)} \Psi_{\boldsymbol{i n}}\left(t_{i}^{\prime}, \overrightarrow{\boldsymbol{r}}^{\prime}, t, \overrightarrow{\boldsymbol{r}}\right) d V^{\prime} .
$$

From the equation above, one can conclude that the net rate of property influx in the general non-moving point $\overrightarrow{\boldsymbol{r}}$ at the given time $t$ is resulted from the impact of the flow of ballistic particles carrying property and converging from the space occupied by the model gas. The equation above calculates the impact in point $\overrightarrow{\boldsymbol{r}}$ at the given time $t$ from all original collisions of converging ballistic particles having trajectories allowing them to target point $\overrightarrow{\boldsymbol{r}}$ at the given time $t$, the original collisions taking place during all preceding dynamic history of the system preceding the given time, i.e. $t_{i}^{\prime}<t$.

2.4 Defining a net rate of total property efflux per unit volume from the general non-moving point at the given time 
To define and formulate the net rate of property efflux per unit volume into surroundings from the general non-moving point $\overrightarrow{\boldsymbol{r}}$ at the given time, $t$, the linear dimensions of the main control volume surrounding point $\overrightarrow{\boldsymbol{r}}$ are selected to be sufficiently small for preventing two and more consecutive collisions of the same particle within the main control volume. We have recognized that the net rate of property efflux per unit volume is formed by a plurality of diverging particles. Each of the plurality of diverging particles is selected from the plurality of particles by the ballistic trajectory having the starting point in the general point of the plurality of non-moving points at the given time. In the following, various terms used in the equations and drawings are listed in Table 2 .

TABLE 2. Terms and symbols used in the equations and drawings.

\begin{tabular}{|c|c|}
\hline Symbol & Short description \\
\hline$t$ & the given time \\
\hline $\overrightarrow{\boldsymbol{r}}$ & $\begin{array}{l}\text { the position of the starting point of a ballistic trajectory of the } \\
\text { diverging particle }\end{array}$ \\
\hline $\overrightarrow{\boldsymbol{r}}^{\prime}$ & $\begin{array}{l}\text { the position of the ending point of a ballistic trajectory of the } \\
\text { diverging particle }\end{array}$ \\
\hline$t_{a}^{\prime}$ & time of positioning the ending point of the diverging particle \\
\hline$n(t, \overrightarrow{\boldsymbol{r}})$ & particle density in the starting point $\overrightarrow{\boldsymbol{r}}$ at the given time $t$ \\
\hline $\overrightarrow{\boldsymbol{u}}(\overrightarrow{\boldsymbol{r}}, t)$ & the mass flow velocity of the model gas in point $\overrightarrow{\boldsymbol{r}}$ at time $t$ \\
\hline$v_{\mathrm{T}}(\overrightarrow{\boldsymbol{r}}, t)$ & $\begin{array}{l}\text { the magnitude of thermal velocity obtained by the diverging } \\
\text { particle in point } \overrightarrow{\boldsymbol{r}} \text { at time } t\end{array}$ \\
\hline $\overrightarrow{\boldsymbol{v}}_{+}\left(t, \overrightarrow{\boldsymbol{r}}, t_{a}^{\prime}\right)$ & $\begin{array}{l}\text { a velocity vector of a diverging particle at the time of positioning } \\
\text { the ending point }\end{array}$ \\
\hline $\overrightarrow{\boldsymbol{r}}_{\boldsymbol{a}}^{c}(t, \overrightarrow{\boldsymbol{r}}, \tilde{t})$ & $\begin{array}{l}\text { the position of a virtual ballistic particle at time } \tilde{t}, \text { which has zero } \\
\text { magnitude of thermal velocity in the starting point } \vec{r} \text { at the time of } \\
\text { original collision } t\end{array}$ \\
\hline $\overrightarrow{\boldsymbol{v}}_{\boldsymbol{a}}^{c}(t, \overrightarrow{\boldsymbol{r}}, \tilde{t})$ & the velocity vector of the virtual ballistic particle at time $\tilde{t}$ \\
\hline$Q_{+}\left(t_{a}^{\prime}, t\right)$ & $\begin{array}{l}\text { the probability of traveling along the ballistic trajectory of the } \\
\text { diverging ballistic trajectory starting at time } t \text { and ending at time } t_{a}^{\prime}\end{array}$ \\
\hline $\boldsymbol{\Psi}\left(t, \overrightarrow{\boldsymbol{r}}, t_{a}^{\prime}, \overrightarrow{\boldsymbol{r}}^{\prime}\right)$ & $\begin{array}{l}\text { property content carried by the diverging particle at the time } t_{a}^{\prime} \text { of } \\
\text { crossing in point } \overrightarrow{\boldsymbol{r}}^{\prime} \text { enclosing control surface } 503\end{array}$ \\
\hline
\end{tabular}

Fig. 5 is a schematic shown to illustrate a method for analytical representation of the net rate of property efflux from a non-moving point in three-dimensional space occupied by the model gas. For clarity, the observer's Cartesian coordinate system 100 is oriented, so the y-axis is along the negative direction of the applied acceleration field 504. In Fig. 5, particle P1 in point $\mathrm{A}$, which is indicated by the position vector $\overrightarrow{\boldsymbol{r}}$ (501) at the given time $t$, diverges from the point source in this point and, in point B, which is indicated by the position vector $\overrightarrow{\boldsymbol{r}}^{\prime}$ (502) at the time $t_{a}^{\prime}$, has velocity $\overrightarrow{\boldsymbol{v}}_{+}$while crossing control surface 503 enclosing the point source in point $\mathrm{A}$. We 


\section{(1) (1) $(9)$}

assume that property

$\Psi\left(t, \overrightarrow{\boldsymbol{r}}, t_{a}^{\prime}, \overrightarrow{\boldsymbol{r}}^{\prime}\right)$ was initially obtained by the particle at the moment of the original collision in point $\overrightarrow{\boldsymbol{r}}$ at time $t$. Specifically, in the point source in point $\overrightarrow{\boldsymbol{r}}$ at time $t$ (point A), each of the diverging particles obtains the thermal velocity of the magnitude of $v_{\mathrm{T}}$ and mass flow velocity $\overrightarrow{\boldsymbol{u}}$ (not shown).

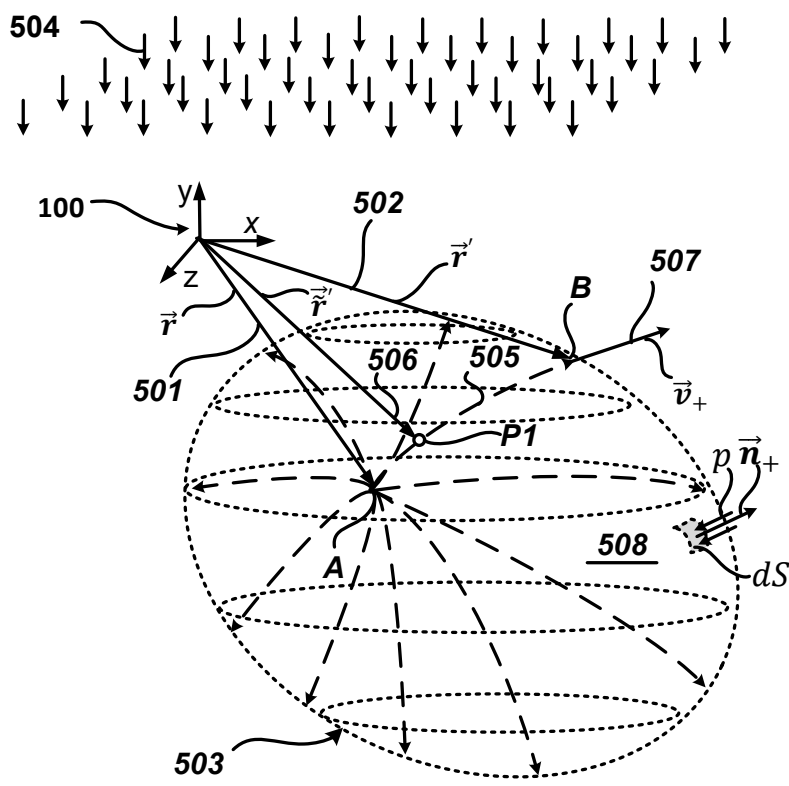

Fig. 5 Schematic three-dimensional view of the model gas system for explaining the ballistic movement of the diverging particle after a collision

We define the net rate of property efflux from the general non-moving point $\overrightarrow{\boldsymbol{r}}$ at the given time $t$ by these four steps:

Step (1) Identifying a trajectory and trajectory characteristics, for each particle diverging from the general non-moving point at the given time

Step (1) includes:

(1) Formulating position vector $\overrightarrow{\widetilde{\boldsymbol{r}}}^{\prime}$ (506) of the particle on trajectory 505 and velocity vector $\overrightarrow{\widetilde{v}}^{\prime}$ (not shown) at time $\tilde{t}$ by applying Equations (39) and (40), respectively, given below:

$$
\overrightarrow{\boldsymbol{r}}^{\prime}=\overrightarrow{\boldsymbol{r}}^{\prime}(t, \overrightarrow{\boldsymbol{r}}, \tilde{t})=v_{\mathrm{T}}(t, \overrightarrow{\boldsymbol{r}}) \overrightarrow{\boldsymbol{n}}_{+\boldsymbol{c}}(\tilde{t}-t)+\overrightarrow{\boldsymbol{r}}_{\boldsymbol{a}}^{c}(t, \overrightarrow{\boldsymbol{r}}, \tilde{t}),
$$

and

$$
{\overrightarrow{\boldsymbol{v}_{+}}}_{+}^{\prime}(\tilde{t})=\overrightarrow{\boldsymbol{v}}_{+}^{\prime}(t, \overrightarrow{\boldsymbol{r}}, \tilde{t})=v_{T}(t, \overrightarrow{\boldsymbol{r}}) \overrightarrow{\boldsymbol{n}}_{+\boldsymbol{c}}+\overrightarrow{\boldsymbol{v}}_{\boldsymbol{a}}^{c}(t, \overrightarrow{\boldsymbol{r}}, \tilde{t}),
$$

where $\overrightarrow{\boldsymbol{n}}_{+\boldsymbol{c}}$ is a unit vector of arbitrary direction with the initial point of origin $\overrightarrow{\boldsymbol{r}}$ at time $t$ and $t_{a}^{\prime} \geq \tilde{t} \geq t$. In Equations (39) and (40) above, $\overrightarrow{\boldsymbol{r}}_{\boldsymbol{a}}^{c}(t, \overrightarrow{\boldsymbol{r}}, \tilde{t})$ and $\overrightarrow{\boldsymbol{v}}_{\boldsymbol{a}}^{\boldsymbol{c}}(t, \overrightarrow{\boldsymbol{r}}, \tilde{t})$ are defined by an appropriate law of motion.

Specifically, when Newton's Law of Motion governs the model gas system, $\overrightarrow{\boldsymbol{r}}_{\boldsymbol{a}}^{\boldsymbol{c}}(t, \overrightarrow{\boldsymbol{r}}, \tilde{t})$ and $\overrightarrow{\boldsymbol{v}}_{\boldsymbol{a}}^{c}(t, \overrightarrow{\boldsymbol{r}}, \tilde{t})$ are defined by Equations (41) and (42), respectively, given below: 


$$
\overrightarrow{\boldsymbol{r}}_{\boldsymbol{a}}^{c}(t, \overrightarrow{\boldsymbol{r}}, \tilde{t})=\overrightarrow{\boldsymbol{r}}+\overrightarrow{\boldsymbol{u}}(t, \overrightarrow{\boldsymbol{r}})(\tilde{t}-t)+\frac{1}{2} \overrightarrow{\boldsymbol{g}}(\tilde{t}-t)^{2}
$$

and

$$
\overrightarrow{\boldsymbol{v}}_{\boldsymbol{a}}^{c}(t, \overrightarrow{\boldsymbol{r}}, \tilde{t})=\overrightarrow{\boldsymbol{u}}(t, \overrightarrow{\boldsymbol{r}})+\overrightarrow{\boldsymbol{g}}(\tilde{t}-t) .
$$

(2) Determining a traveling time needed, for each particle diverging from a non-moving point $\overrightarrow{\boldsymbol{r}}$ at the given time $t$ to cross a control surface enclosing point $\overrightarrow{\boldsymbol{r}}$ in point $\overrightarrow{\boldsymbol{r}}^{\prime}$ of the control surface. It can be done by solving Equation (39) in which $\tilde{t}=t_{a}^{\prime}$. Specifically, when Newton's Laws of Motion govern the motion of the particles, then it can be done by resolving the equation of projectile motion, Equation (43) given below, with respect to the ballistic traveling time $\varphi_{+}$

$$
\overrightarrow{\boldsymbol{r}}^{\prime}=\overrightarrow{\boldsymbol{r}}+\left[v_{\mathrm{T}}(t, \overrightarrow{\boldsymbol{r}}) \overrightarrow{\boldsymbol{n}}_{+\boldsymbol{c}}+\overrightarrow{\boldsymbol{u}}(t, \overrightarrow{\boldsymbol{r}})\right] \varphi_{+}+\frac{1}{2} \overrightarrow{\mathbf{g}} \varphi_{+}{ }^{2},
$$

which is obtained by assigning $\tilde{t}=t_{a}^{\prime}$ and substitution of $\varphi_{+}=t_{a}^{\prime}-t$ in Equation (39) and rearrangement of the terms. Traveling time $\varphi_{+}$is needed to indicate a time $t_{a}^{\prime}$ at which the departing particle has reached a point $\overrightarrow{\boldsymbol{r}}^{\prime}$ on control surface 503 enclosing point $\overrightarrow{\boldsymbol{r}}$.

(3) Defining an appropriate instant unit vector directing thermal velocity component along the diverging ballistic trajectory of each particle diverging from the general non-moving point $\overrightarrow{\boldsymbol{r}}$ at time $t$ includes:

presenting Equation (43) in the following form:

$$
\varphi_{+} v_{\mathrm{T}} \overrightarrow{\boldsymbol{n}}_{+\boldsymbol{c}}=\overrightarrow{\boldsymbol{r}}^{\prime}-\overrightarrow{\boldsymbol{r}}_{\boldsymbol{a}}^{c},
$$

where $\overrightarrow{\boldsymbol{n}}_{+\boldsymbol{c}}$ is a unit vector and $\overrightarrow{\boldsymbol{r}}_{\boldsymbol{a}}^{c}$ (not shown) is a virtual position of the particle leaving point $\overrightarrow{\boldsymbol{r}}$ at time $t$, which would have zero magnitude of the thermal velocity and is observed at time $t_{a}^{\prime}$ and, where a model gas system is governed by Newton's Laws of Motion, is expressed as:

$$
\overrightarrow{\boldsymbol{r}}_{\boldsymbol{a}}^{\boldsymbol{c}}=\overrightarrow{\boldsymbol{r}}+\overrightarrow{\boldsymbol{u}}(t, \overrightarrow{\boldsymbol{r}}) \varphi_{+}+\frac{1}{2} \overrightarrow{\mathbf{g}} \varphi_{+}^{2}
$$

and deriving $\overrightarrow{\boldsymbol{n}}_{+\boldsymbol{c}}$ from Equation (44), which is given as

$$
\overrightarrow{\boldsymbol{n}}_{+c}=\frac{\vec{r}^{\prime}-\overrightarrow{\boldsymbol{r}}_{a}^{c}}{\left|\overrightarrow{\boldsymbol{r}}^{\prime}-\overrightarrow{\boldsymbol{r}}_{a}^{c}\right|}
$$

where $\overrightarrow{\boldsymbol{n}}_{+\boldsymbol{c}}$ is interpreted as a unit vector of origin $\overrightarrow{\boldsymbol{r}}_{\boldsymbol{a}}^{c}$ at time $t_{a}^{\prime}$, which directs the vector of the thermal velocity component and where $t_{a}^{\prime}$ is the time of positioning in point $\overrightarrow{\boldsymbol{r}}^{\prime}$, which is on the control surface 503.

(4) Defining the velocity vector $\overrightarrow{\boldsymbol{v}}_{+}$, (507) of each particle at the moment of crossing the control surface in point $\overrightarrow{\boldsymbol{r}}^{\prime}$ includes, where a model gas system is governed by Newton's Laws of Motion, representing $\overrightarrow{\boldsymbol{v}}_{+}$by applying Equation (47) as given below:

$$
\overrightarrow{\boldsymbol{v}}_{+}\left(t, \overrightarrow{\boldsymbol{r}}, t_{a}^{\prime}, \overrightarrow{\boldsymbol{r}}^{\prime}\right)=v_{\mathrm{T}}(t, \overrightarrow{\boldsymbol{r}}) \overrightarrow{\boldsymbol{n}}_{+\boldsymbol{c}}+\overrightarrow{\boldsymbol{u}}(t, \overrightarrow{\boldsymbol{r}})+\overrightarrow{\mathbf{g}} \varphi_{+},
$$

which is obtained by assigning $\tilde{t}=t_{a}^{\prime}$ and substitution of $t_{a}^{\prime}-t=\varphi_{+}$in Equation (40).

Step (2) Defining the probability of traveling along the ballistic trajectory from the general non-moving point $\overrightarrow{\boldsymbol{r}}$ at time $t$ to one of the plurality of points in space surrounding the general non-moving point 


\section{(c) $(1)(\Theta \Theta$}

Step (2) includes representing the probability $Q_{+}\left(t_{a}^{\prime}, t\right)$ by applying Equation (48) given below:

$$
Q_{+}\left(t_{a}^{\prime}, t\right)=Q_{+}\left(0, \varphi_{i+}\right)=\exp \left(-\int_{t}^{t_{a}^{\prime}} P_{c}\left(\overrightarrow{\widetilde{r}}^{\prime}(\tilde{t})\right) v_{r e l}\left(\overrightarrow{\widetilde{r}}^{\prime}(\tilde{t})\right) \mathrm{d} \tilde{t}\right),
$$

where $t_{a}^{\prime}=t+\varphi_{+}$is a time of the particle positioning in point $\overrightarrow{\boldsymbol{r}}^{\prime}, P_{c}\left(\overrightarrow{\widetilde{\boldsymbol{r}}}^{\prime}(\tilde{t})\right)$ is the average number of collisions per unit length in the trajectory point $\overrightarrow{\widetilde{\boldsymbol{r}}}^{\prime}(\tilde{t}), v_{\text {rel }}\left(\overrightarrow{\widetilde{\boldsymbol{r}}}^{\prime}(\tilde{t})\right)$ is an average magnitude of the velocity of the particle traveling along the diverging ballistic trajectory with respect to a nearby passed particle in the trajectory point $\overrightarrow{\widetilde{r}}^{\prime}(\tilde{t})$.

Step (3) Defining vector/tensor field of property flux $\boldsymbol{J}_{r}^{\Psi}$ along one of the ballistic trajectories of a diverging particle in point $\overrightarrow{\boldsymbol{r}}^{\prime}$

Step (3) includes representing the property vector flux $\boldsymbol{J}_{\boldsymbol{r}}^{\Psi}$ by applying Equation (49) as given below:

$$
\boldsymbol{J}_{\boldsymbol{r}}^{\boldsymbol{\Psi}}=\frac{1}{2} n(t, \overrightarrow{\boldsymbol{r}}) Q_{+}\left(t_{a}^{\prime}, t\right) \overrightarrow{\boldsymbol{v}}_{+}\left(t, \overrightarrow{\boldsymbol{r}}, t_{a}^{\prime}, \overrightarrow{\boldsymbol{r}}^{\prime}\right) \Psi\left(t, \overrightarrow{\boldsymbol{r}}, t_{a}^{\prime}, \overrightarrow{\boldsymbol{r}}^{\prime}\right) .
$$

Step (4) Defining the net rate of property efflux per unit volume $\boldsymbol{B}_{\text {out }}^{\Psi_{\text {IS }}}$ from a point source positioned in the general non-moving point $\overrightarrow{\boldsymbol{r}}$ at the given time $t$

Step (4) includes representing $\boldsymbol{B}_{\text {out }}^{\Psi_{\mathbf{L}} \text {.FS }}$ by applying Equation (50) as given below:

$$
\boldsymbol{B}_{\text {out }}^{\Psi_{-} \mathrm{FS}}(\vec{r}, t)=\frac{1}{2}\left\{\boldsymbol{\nabla} \cdot\left[n(t, \overrightarrow{\boldsymbol{r}}) Q_{+}\left(t_{a}^{\prime}, t\right) \overrightarrow{\boldsymbol{v}}_{+}\left(t, \overrightarrow{\boldsymbol{r}}, t_{a}^{\prime}, \overrightarrow{\boldsymbol{r}}^{\prime}\right) \boldsymbol{\Psi}\left(t, \overrightarrow{\boldsymbol{r}}, t_{a}^{\prime}, \overrightarrow{\boldsymbol{r}}^{\prime}\right)\right]\right\}_{\overrightarrow{\boldsymbol{r}}^{\prime} \rightarrow \overrightarrow{\boldsymbol{r}}},
$$

which is obtained by executing the divergence operator $\boldsymbol{\nabla} \cdot$ to the vector field of Equation (49) and by shrinking the volume of control volume 508 confined by the control surface 503 to an infinitely small volume, i.e., $\overrightarrow{\boldsymbol{r}}^{\prime} \rightarrow \overrightarrow{\boldsymbol{r}}$, which also leads to the limit $\overrightarrow{\boldsymbol{r}}_{\boldsymbol{a}}^{\boldsymbol{c}} \rightarrow \overrightarrow{\boldsymbol{r}}$.

2.5 Analytical representation of a general integro-differential form of property balance equation in the three-dimensional model gas system

The integro-differential form of property balance equation is formulated by Equation (51) given below, which is obtained by substitution of Equations (38) and (50) in Equation (8):

$$
\begin{gathered}
\frac{\partial}{\partial t}[n(t, \vec{r}) \Psi(t, \vec{r})]+\frac{1}{2}\left\{\boldsymbol{\nabla} \cdot\left[n(t, \overrightarrow{\boldsymbol{r}}) Q_{+}\left(t_{a}^{\prime}, t\right) \overrightarrow{\boldsymbol{v}}_{+}\left(t, \overrightarrow{\boldsymbol{r}}, t_{a}^{\prime}, \overrightarrow{\boldsymbol{r}}^{\prime}\right) \boldsymbol{\Psi}\left(t, \overrightarrow{\boldsymbol{r}}, t_{a}^{\prime}, \overrightarrow{\boldsymbol{r}}^{\prime}\right)\right]\right\}_{\overrightarrow{\boldsymbol{r}}^{\prime} \rightarrow \overrightarrow{\boldsymbol{r}}}, \\
=-\boldsymbol{\nabla} \cdot \iiint_{\mathrm{V}} \frac{1}{4 \pi} \frac{1}{\left|\overrightarrow{\boldsymbol{r}}-\overrightarrow{\boldsymbol{r}}_{\boldsymbol{i}}^{c}\right|^{2}} Q_{i}\left(t, t_{i}^{\prime}\right) Z_{V}\left(t_{i}^{\prime}, \overrightarrow{\boldsymbol{r}}^{\prime}\right) \frac{\overrightarrow{\boldsymbol{v}}\left(t_{i}^{\prime}, \overrightarrow{\boldsymbol{r}}^{\prime}, t, \overrightarrow{\boldsymbol{r}}^{\prime}\right)}{v_{T}\left(t_{i}^{\prime}, \overrightarrow{\boldsymbol{r}}^{\prime}\right)} \Psi_{i \boldsymbol{n}}\left(t_{i}^{\prime}, \overrightarrow{\boldsymbol{r}}^{\prime}, t, \overrightarrow{\boldsymbol{r}}\right) d V^{\prime},
\end{gathered}
$$

Here point $\overrightarrow{\boldsymbol{r}}$ is not included in integration for converging ballistic particles. It implies that any singularity in the right-hand of the equation above is excluded, so Equation (51) defines $\Psi$ at $t \geq 0$ as an implicit function of $\overrightarrow{\boldsymbol{r}}$ on $\mathbb{R}^{3}$, i.e. $\overrightarrow{\boldsymbol{r}} \in \mathbb{R}^{3}$.

The analytical representation of the general integro-differential form of property balance equation shown by Equation (51) is generally valid for any homogeneous fluid system with any configuration of the external field of force. 
Remark that the integro-differential property balance equation needs to be formed for each unknown property/variable so that the number of equations in a system of balance equations is sufficient to determine each of the unknown properties characterizing the model gas flow. In the following, we provide general governing integro-differential forms of mass balance, momentum balance, and energy balance equations.

2.5.1 Analytical representation of a general integro-differential form of mass balance equation in the three-dimensional model gas system

To formulate a general integro-differential form of mass balance equation in a given nonmoving point of space occupied by the model at a given time, we will modify Equation (51) by assigning:

$$
\Psi=\Psi_{\text {in }}=1
$$

Then, we obtain the following general integro-differential form of the mass balance equation

$$
\begin{aligned}
\frac{\partial}{\partial t} & {[n(t, \vec{r})]+\frac{1}{2}\left\{\boldsymbol{\nabla} \cdot\left[n(t, \overrightarrow{\boldsymbol{r}}) Q_{+}\left(t_{a}^{\prime}, t\right) \overrightarrow{\boldsymbol{v}}_{+}\left(t, \overrightarrow{\boldsymbol{r}}, t_{a}^{\prime}, \overrightarrow{\boldsymbol{r}}^{\prime}\right)\right]\right\}_{\overrightarrow{\boldsymbol{r}}^{\prime} \rightarrow \overrightarrow{\boldsymbol{r}}}, } \\
& =-\boldsymbol{\nabla} \cdot \iiint_{\mathrm{V}} \frac{1}{4 \pi} \frac{1}{\left|\overrightarrow{\boldsymbol{r}}-\overrightarrow{\boldsymbol{r}}_{\boldsymbol{i}}^{c}\right|^{2}} Q_{i}\left(t, t_{i}^{\prime}\right) Z_{V}\left(t_{i}^{\prime}, \overrightarrow{\boldsymbol{r}}^{\prime}\right) \frac{\overrightarrow{\boldsymbol{v}}\left(t_{i}^{\prime}, \overrightarrow{\boldsymbol{r}}^{\prime}, t, \overrightarrow{\boldsymbol{r}}\right)}{v_{T}\left(t_{i}^{\prime}, \overrightarrow{\boldsymbol{r}}^{\prime}\right)} d V^{\prime} .
\end{aligned}
$$

2.5.2 Analytical representation of a general integro-differential form of momentum balance equation in the three-dimensional model gas system

To formulate a general integro-differential form of momentum balance equation in a given non-moving point of space occupied by the model at a given time, we will modify Equation (51) by assigning:

$$
\Psi\left(t, \overrightarrow{\boldsymbol{r}}, t_{a}^{\prime}, \overrightarrow{\boldsymbol{r}}^{\prime}\right)=\overrightarrow{\boldsymbol{v}}_{+}\left(t, \overrightarrow{\boldsymbol{r}}, t_{a}^{\prime}, \overrightarrow{\boldsymbol{r}}^{\prime}\right)
$$

in the left-hand of the equation and

$$
\Psi_{\text {in }}\left(t_{i}^{\prime}, \overrightarrow{\boldsymbol{r}}^{\prime}, t, \overrightarrow{\boldsymbol{r}}\right)=\overrightarrow{\boldsymbol{v}}\left(t_{i}^{\prime}, \overrightarrow{\boldsymbol{r}}^{\prime}, t, \overrightarrow{\boldsymbol{r}}\right)
$$

in the right-hand of the equation. Then we obtain:

$$
\begin{gathered}
\left\{m \frac{\partial}{\partial t}\left[n(t, \vec{r}) \overrightarrow{\boldsymbol{v}}_{+}\left(t, \overrightarrow{\boldsymbol{r}}, t_{a}^{\prime}, \overrightarrow{\boldsymbol{r}}^{\prime}\right)\right]\right\}_{\overrightarrow{\boldsymbol{r}}^{\prime} \rightarrow \overrightarrow{\boldsymbol{r}}} \\
+\frac{1}{2}\left\{\boldsymbol{\nabla} \cdot\left[m n(t, \overrightarrow{\boldsymbol{r}}) Q_{+}\left(t_{a}^{\prime}, t\right) \overrightarrow{\boldsymbol{v}}_{+}\left(t, \overrightarrow{\boldsymbol{r}}, t_{a}^{\prime}, \overrightarrow{\boldsymbol{r}}^{\prime}\right) \overrightarrow{\boldsymbol{v}}_{+}\left(t, \overrightarrow{\boldsymbol{r}}_{,} t_{a}^{\prime}, \overrightarrow{\boldsymbol{r}}^{\prime}\right)\right]\right\}_{\overrightarrow{\boldsymbol{r}}^{\prime} \rightarrow \overrightarrow{\boldsymbol{r}}^{\prime}}+\nabla p(t, \overrightarrow{\boldsymbol{r}}) \\
=-m \boldsymbol{\nabla} \cdot \iiint_{\mathrm{V}} \frac{1}{4 \pi} \frac{1}{\left|\overrightarrow{\boldsymbol{r}}-\overrightarrow{\boldsymbol{r}}_{\boldsymbol{i}}^{c}\right|^{2}} Q_{i}\left(t, t_{i}^{\prime}\right) Z_{V}\left(t_{i}^{\prime}, \overrightarrow{\boldsymbol{r}}^{\prime}\right) \frac{\overrightarrow{\boldsymbol{v}}\left(t_{i}^{\prime}, \overrightarrow{\boldsymbol{r}}^{\prime}, t, \overrightarrow{\boldsymbol{r}}\right) \overrightarrow{\boldsymbol{v}}_{+}\left(t, \overrightarrow{\boldsymbol{r}}, t_{a}^{\prime}, \overrightarrow{\boldsymbol{r}}^{\prime}\right)}{v_{T}\left(t_{i}^{\prime}, \overrightarrow{\boldsymbol{r}}^{\prime}\right)} d V^{\prime} .
\end{gathered}
$$

In the equation above, we also introduce a term of the pressure force exerted on its surroundings in point $\overrightarrow{\boldsymbol{r}}$ at time $t$, which may appear because of a non-uniform pressure applied to a bounded system.

2.5.3 Analytical representation of a general integro-differential form of energy balance equation in the three-dimensional model gas system 
To formulate a general integro-differential form of energy balance equation in a given nonmoving point of space occupied by the model at a given time, we will modify Equation (51) by assigning:

$$
\Psi\left(t, \overrightarrow{\boldsymbol{r}}, t_{a}^{\prime}, \overrightarrow{\boldsymbol{r}}^{\prime}\right)=\frac{1}{2} \overrightarrow{\boldsymbol{v}}_{+}\left(t, \overrightarrow{\boldsymbol{r}}, t_{a}^{\prime}, \overrightarrow{\boldsymbol{r}}^{\prime}\right) \cdot \overrightarrow{\boldsymbol{v}}_{+}\left(t, \overrightarrow{\boldsymbol{r}}, t_{a}^{\prime}, \overrightarrow{\boldsymbol{r}}^{\prime}\right)
$$

in the left-hand of the equation and

$$
\Psi_{\text {in }}\left(t_{i}^{\prime}, \overrightarrow{\boldsymbol{r}}^{\prime}, t, \overrightarrow{\boldsymbol{r}}\right)=\frac{1}{2} \overrightarrow{\boldsymbol{v}}\left(t_{i}^{\prime}, \overrightarrow{\boldsymbol{r}}^{\prime}, t, \overrightarrow{\boldsymbol{r}}\right) \cdot \overrightarrow{\boldsymbol{v}}\left(t_{i}^{\prime}, \overrightarrow{\boldsymbol{r}}^{\prime}, t, \overrightarrow{\boldsymbol{r}}\right)
$$

in the right-hand of the equation. Then we obtain:

$$
\begin{gathered}
\left\{\frac{\partial}{\partial t}\left[n(t, \vec{r}) \overrightarrow{\boldsymbol{v}}_{+} \cdot \overrightarrow{\boldsymbol{v}}_{+}\right]\right\}_{\overrightarrow{\boldsymbol{r}}^{\prime} \rightarrow \overrightarrow{\boldsymbol{r}}^{\prime}} \\
+\frac{1}{2}\left\{\boldsymbol{\nabla} \cdot\left[n(t, \overrightarrow{\boldsymbol{r}}) Q_{+}\left(t_{a}^{\prime}, t\right) \overrightarrow{\boldsymbol{v}}_{+}\left(t, \overrightarrow{\boldsymbol{r}}, t_{a}^{\prime}, \overrightarrow{\boldsymbol{r}}^{\prime}\right) \overrightarrow{\boldsymbol{v}}_{+} \cdot \overrightarrow{\boldsymbol{v}}_{+}+\frac{1}{m} p(t, \overrightarrow{\boldsymbol{r}}) \overrightarrow{\boldsymbol{v}}_{+}\right]\right\}_{\overrightarrow{\boldsymbol{r}}^{\prime} \rightarrow \overrightarrow{\boldsymbol{r}}} \\
=-\boldsymbol{\nabla} \cdot \iiint_{\mathrm{V}} \frac{1}{4 \pi} \frac{1}{\left|\overrightarrow{\boldsymbol{r}}-\overrightarrow{\boldsymbol{r}}_{\boldsymbol{i}}^{c}\right|^{2}} Q_{i}\left(t, t_{i}^{\prime}\right) Z_{V}\left(t_{i}^{\prime}, \overrightarrow{\boldsymbol{r}}^{\prime}\right) \frac{\overrightarrow{\boldsymbol{v}}\left(t_{i}^{\prime}, \overrightarrow{\boldsymbol{r}}^{\prime}, t, \overrightarrow{\boldsymbol{r}}\right)}{v_{T}\left(t_{i}^{\prime}, \overrightarrow{\boldsymbol{r}}^{\prime}\right)} \overrightarrow{\boldsymbol{v}}\left(t_{i}^{\prime}, \overrightarrow{\boldsymbol{r}}^{\prime}, t, \overrightarrow{\boldsymbol{r}}\right) \cdot \overrightarrow{\boldsymbol{v}}\left(t_{i}^{\prime}, \overrightarrow{\boldsymbol{r}}^{\prime}, t, \overrightarrow{\boldsymbol{r}}\right) d V^{\prime} .
\end{gathered}
$$

In the second left-term of the equation above, we also introduced a term of the pressure force work on its surroundings in point $\overrightarrow{\boldsymbol{r}}$ at time $t$, which may appear because of a non-uniform pressure applied to the system.

\section{Integro-Differential Forms of Mass and Momentum Balance Equations in Collision- Dominated Flow Regime}

We have recognized that, in the majority of real conditions on Earth and near-Earth space, first, the acceleration field $\overrightarrow{\mathbf{g}}$ applied to each of the plurality of the model gas particles is approximately a constant, namely:

$$
\overrightarrow{\mathbf{g}}=\text { constant; }
$$

second, for each of the model gas particles, the average magnitude of the thermal velocity component, $v_{\mathrm{T}}$, is much higher than the magnitude of a mass-flow velocity component $|\overrightarrow{\boldsymbol{u}}|$, namely:

$$
|\overrightarrow{\boldsymbol{u}}| \ll v_{\mathrm{T}} ;
$$

third, in the collision-dominated flow regime, the magnitude of velocity gained or lost because of interaction with the acceleration field during the ballistic traveling time $\varphi_{i 0}$ is insignificant in comparison with the thermal velocity component $v_{\mathrm{T}}$, namely:

$$
\left|\vec{g} \varphi_{i 0}\right| \ll v_{T}
$$

fourth, in the collision-dominated flow regime, the relative change of any property value or any parameter characterizing the model gas $\Sigma$ is insignificant during the period between collisions $\frac{\mathbf{1}}{P_{c} v_{\text {rel }}}$ in a given point of the model gas, which is expressed:

$$
\frac{1}{P_{c} v_{r e l}} \frac{\left|\frac{d}{d t} \Sigma\right|}{|\Sigma|} \ll 1
$$


and fifth, in the collision-dominated flow regime, the relative change of any property value or any parameter characterizing the model gas is insignificant on the length scale of the average distance between the model gas particles $\frac{1}{P_{c}}$, which is expressed:

$$
\frac{1}{P_{c}} \frac{|\nabla \Sigma|}{|\Sigma|} \ll 1
$$

We also note here that since point $\overrightarrow{\boldsymbol{r}}$ is excluded from integration, then, in the domain of integration $\Omega$ in which $\overrightarrow{\boldsymbol{r}}^{\prime} \neq \overrightarrow{\boldsymbol{r}}$, the operation of differentiation regarding a parameter $\overrightarrow{\boldsymbol{r}}$ is interchangeable with the operation of integration over some other variable $\overrightarrow{\boldsymbol{r}}^{\prime}$. In addition, these functions or approximations for functions involving in calculations according to Equations (53), (56), and (59) are formulated:

(1) Approximating $\overrightarrow{\boldsymbol{v}}_{+}\left(t, t_{a}^{\prime}, \overrightarrow{\boldsymbol{r}}^{\prime}\right)$ as

$$
\overrightarrow{\boldsymbol{v}}_{+}\left(t, \overrightarrow{\boldsymbol{r}}, t_{a}^{\prime}, \overrightarrow{\boldsymbol{r}}^{\prime}\right) \cong \overrightarrow{\boldsymbol{v}}_{+0}\left(t, \overrightarrow{\boldsymbol{r}}, t_{a 0}^{\prime}, \overrightarrow{\boldsymbol{r}}^{\prime}\right)=v_{\mathrm{T}}(t, \overrightarrow{\boldsymbol{r}}) \overrightarrow{\boldsymbol{n}}_{+\mathbf{0}}+\overrightarrow{\boldsymbol{u}}(t, \overrightarrow{\boldsymbol{r}})+\overrightarrow{\boldsymbol{g}} \varphi_{+0},
$$

where $\overrightarrow{\boldsymbol{n}}_{+\mathbf{0}}$ is the unit vector of arbitrary direction from the point $\overrightarrow{\boldsymbol{r}}$, which is approximated from $\overrightarrow{\boldsymbol{n}}_{+}$as

$$
\overrightarrow{\boldsymbol{n}}_{+} \cong \overrightarrow{\boldsymbol{n}}_{+\mathbf{0}}=\frac{\overrightarrow{\boldsymbol{r}}^{\prime}-\overrightarrow{\boldsymbol{r}}}{\left|\overrightarrow{\boldsymbol{r}}^{\prime}-\overrightarrow{\boldsymbol{r}}\right|},
$$

and $\varphi_{+0}$ is the ballistic traveling time, which is approximated from $\varphi_{+}$as

$$
\varphi_{+} \cong \varphi_{+0}=\frac{\left|\overrightarrow{\boldsymbol{r}}^{\prime}-\overrightarrow{\boldsymbol{r}}\right|}{v_{T}(\overrightarrow{\boldsymbol{r}}, t)} .
$$

(2) Approximating $Q_{+}\left(t_{a}^{\prime}, t\right)$ as

$$
Q_{+}\left(t_{a}^{\prime}, t\right) \cong Q_{+0}\left(t_{a 0}^{\prime}, t\right)=\exp \left(-\int_{t}^{t_{a 0}^{\prime}} P_{c}\left(\overrightarrow{\widetilde{\boldsymbol{r}}}^{\prime}(\tilde{t})\right) v_{r e l}\left(\overrightarrow{\widetilde{\boldsymbol{r}}}^{\prime}(\tilde{t})\right) \mathrm{d} \tilde{t}\right),
$$

where $t_{a 0}^{\prime}$ is a time of the particle positioning in point $\overrightarrow{\boldsymbol{r}}^{\prime}$, which is approximated from $t_{a}^{\prime}$ as

$$
t_{a}^{\prime} \cong t_{a 0}^{\prime}=t+\varphi_{+0} .
$$

(3) For $\overrightarrow{\boldsymbol{r}}^{\prime} \rightarrow \overrightarrow{\boldsymbol{r}}$, executing $\nabla Q_{+0}\left(t_{a 0}^{\prime}, t\right)$ as

(4) Approximating $\overrightarrow{\boldsymbol{v}}\left(t_{i}^{\prime}, \overrightarrow{\boldsymbol{r}}^{\prime}, t, \overrightarrow{\boldsymbol{r}}\right)$ as

$$
\overrightarrow{\boldsymbol{v}}\left(t_{i}^{\prime}, \overrightarrow{\boldsymbol{r}}^{\prime}, t, \overrightarrow{\boldsymbol{r}}\right) \cong \overrightarrow{\boldsymbol{v}}\left(t_{i 0}^{\prime}, \overrightarrow{\boldsymbol{r}}^{\prime}, t, \overrightarrow{\boldsymbol{r}}\right)=v_{T}\left(t_{i 0}^{\prime}, \overrightarrow{\boldsymbol{r}}^{\prime}\right) \overrightarrow{\boldsymbol{n}}_{i \mathbf{0}}+\overrightarrow{\boldsymbol{u}}\left(t_{i 0}^{\prime}, \overrightarrow{\boldsymbol{r}}^{\prime}\right)+\overrightarrow{\boldsymbol{g}} \varphi_{i 0},
$$

where $\overrightarrow{\boldsymbol{n}}_{\boldsymbol{i} \mathbf{0}}$ is the unit vector of arbitrary direction from the point $\overrightarrow{\boldsymbol{r}}^{\prime}$, which is approximated from $\overrightarrow{\boldsymbol{n}}_{\boldsymbol{i}}$ as

$$
\overrightarrow{\boldsymbol{n}}_{\boldsymbol{i}} \cong \overrightarrow{\boldsymbol{n}}_{\boldsymbol{i} \mathbf{0}}=\frac{\overrightarrow{\boldsymbol{r}}-\overrightarrow{\boldsymbol{r}}^{\prime}}{\left|\overrightarrow{\boldsymbol{r}}-\overrightarrow{\boldsymbol{r}}^{\prime}\right|}
$$

and $\varphi_{i 0}$ is the ballistic traveling time, which is approximated from $\varphi_{i}$ as

$$
\varphi_{i} \cong \varphi_{i 0}=\frac{\left|\overrightarrow{\boldsymbol{r}}-\overrightarrow{\boldsymbol{r}}^{\prime}\right|}{v_{T}\left(t_{i}^{\prime}, \vec{r}^{\prime}\right)} .
$$

(5) Approximating $Q_{i}\left(t, t_{i}^{\prime}\right)$ as

$$
Q_{i}\left(t, t_{i}^{\prime}\right) \cong Q_{i 0}\left(t, t_{i 0}^{\prime}\right)=\exp \left(-\int_{t_{i 0}^{\prime}}^{t} P_{c}(\overrightarrow{\widetilde{r}}(\tilde{t})) v_{r e l}(\overrightarrow{\widetilde{r}}(\tilde{t})) \mathrm{d} \tilde{t}\right),
$$

where $t_{i 0}^{\prime}$ is a time of the particle positioning in point $\overrightarrow{\boldsymbol{r}}^{\prime}$, which is approximated from $t_{i}^{\prime}$ as 


$$
t_{i}^{\prime} \cong t_{i 0}^{\prime}=t-\varphi_{i 0}
$$

(6) Approximating $\nabla Q_{i 0}\left(t, t_{i 0}^{\prime}\right)$ as

$$
\nabla Q_{i 0}\left(t, t_{i 0}^{\prime}\right) \cong-Q_{i 0}\left(t, t_{i 0}^{\prime}\right) P_{c}(t, \overrightarrow{\boldsymbol{r}}) \boldsymbol{v}_{\boldsymbol{r e l}}(t, \overrightarrow{\boldsymbol{r}}) \frac{\overrightarrow{\boldsymbol{n}}_{i 0}}{v_{T}\left(t_{i}^{\prime}, \vec{r}^{\prime}\right)}
$$

(7) Approximating $\overrightarrow{\boldsymbol{r}}_{\boldsymbol{i}}^{\boldsymbol{c}}$ as

$$
\overrightarrow{\boldsymbol{r}}_{i}^{c} \cong \overrightarrow{\boldsymbol{r}}^{\prime}
$$

(8) Representing $\mathbf{G}\left(\overrightarrow{\boldsymbol{r}}, \overrightarrow{\boldsymbol{r}}^{\prime}\right)$, a first vector derivative of the Green function with no boundary conditions, as

$$
\mathbf{G}\left(\overrightarrow{\boldsymbol{r}}, \overrightarrow{\boldsymbol{r}}^{\prime}\right)=\frac{1}{4 \pi} \frac{\overrightarrow{\boldsymbol{r}}-\overrightarrow{\boldsymbol{r}}^{\prime}}{\left|\overrightarrow{\boldsymbol{r}}-\overrightarrow{\boldsymbol{r}}^{\prime}\right|^{3}}
$$

which has the following property:

$$
\boldsymbol{\nabla} \cdot \mathbf{G}\left(\overrightarrow{\boldsymbol{r}}, \overrightarrow{\boldsymbol{r}}^{\prime}\right)=\delta\left(\overrightarrow{\boldsymbol{r}}-\overrightarrow{\boldsymbol{r}}^{\prime}\right) .
$$

Considering that governing equations require satisfaction of the balance of any model gas property at any time, these analytical representations of approximations for mass balance, momentum balance, and energy balance are provided.

\subsection{Analytical representation of an integro-differential form of the mass balance equation in the} collision-dominated flow regime

To formulate an integro-differential form of mass balance equation in the collisiondominated flow regime, which applies to a given non-moving point of space occupied by the model at a given time, we will modify Equation (53) as follows:

(1) Substituting approximations from Equation (65) to Equation (78) in Equation (53) and executing vector differentiation; and

(2) Executing limit $\overrightarrow{\boldsymbol{r}}^{\prime} \rightarrow \overrightarrow{\boldsymbol{r}}$ in the second left-hand term of the resulting approximated integro-differential mass balance equation.

We obtain the following approximated integro-differential form of mass balance equation:

$$
\begin{aligned}
& \frac{1}{P_{c}(t, \overrightarrow{\boldsymbol{r}}) v_{r e l}(t, \overrightarrow{\boldsymbol{r}})}\left\{\frac{\partial}{\partial t}[n(t, \overrightarrow{\boldsymbol{r}})]+Z_{V}(t, \overrightarrow{\boldsymbol{r}})+\frac{1}{2} \boldsymbol{\nabla} \cdot[n(t, \vec{r}) \overrightarrow{\boldsymbol{u}}(t, \overrightarrow{\boldsymbol{r}})]\right\} \\
& \cong-\iiint_{\mathrm{V}} Q_{i 0}\left(t, t_{i 0}^{\prime}\right) Z_{V}\left(t_{i 0}^{\prime}, \overrightarrow{\boldsymbol{r}}^{\prime}\right) \delta\left(\overrightarrow{\boldsymbol{r}}-\overrightarrow{\boldsymbol{r}}^{\prime}\right) d V^{\prime} \\
& +\iiint_{\mathrm{V}} Q_{i 0}\left(t, t_{i 0}^{\prime}\right) \frac{1}{4 \pi} \frac{1}{\left|\overrightarrow{\boldsymbol{r}}-\overrightarrow{\boldsymbol{r}}^{\prime}\right|^{2}} \frac{z_{V}\left(t_{i 0}^{\prime}, \overrightarrow{\boldsymbol{r}}^{\prime}\right)}{v_{T}\left(t_{i 0}^{\prime}, \vec{r}^{\prime}\right)} d V^{\prime} \\
& -\frac{1}{P_{c}(t, \overrightarrow{\boldsymbol{r}}) v_{\text {rel }}(t, \overrightarrow{\boldsymbol{r}})} \iiint_{\mathrm{V}} Q_{i 0}\left(t, t_{i 0}^{\prime}\right) \frac{1}{4 \pi} \frac{1}{\left|\overrightarrow{\boldsymbol{r}}-\overrightarrow{\boldsymbol{r}}^{\prime}\right|^{2}} \frac{1}{v_{T}\left(t_{i 0}^{\prime}, \overrightarrow{\boldsymbol{r}}^{\prime}\right)} \frac{d}{d t_{i 0}^{\prime}} Z_{V}\left(t_{i 0}^{\prime}, \overrightarrow{\boldsymbol{r}}^{\prime}\right) d V^{\prime} \\
& +\iiint_{\mathrm{V}} Z_{V}\left(t_{i 0}^{\prime}, \overrightarrow{\boldsymbol{r}}^{\prime}\right) Q_{i 0}\left(t, t_{i 0}^{\prime}\right) \mathbf{G}\left(\overrightarrow{\boldsymbol{r}}, \overrightarrow{\boldsymbol{r}}^{\prime}\right) \cdot \overrightarrow{\boldsymbol{u}}\left(t_{i 0}^{\prime}, \overrightarrow{\boldsymbol{r}}^{\prime}\right) \frac{1}{v_{T}^{2}\left(t_{i 0}^{\prime}, \overrightarrow{\boldsymbol{r}}^{\prime}\right)} d V^{\prime} \\
& +\frac{\mathbf{1}}{P_{c}(t, \overrightarrow{\boldsymbol{r}}) \boldsymbol{v}_{\boldsymbol{r e l}}(t, \overrightarrow{\boldsymbol{r}})} \iiint_{\mathrm{V}} Q_{i 0} \frac{d}{d t_{i 0}^{\prime}}\left[\frac{z_{V}\left(t_{i 0}^{\prime}, \overrightarrow{\boldsymbol{r}}^{\prime}\right)}{v_{T}\left(t_{i 0}^{\prime}, \overrightarrow{\boldsymbol{r}}^{\prime}\right)} \overrightarrow{\boldsymbol{u}}\left(t_{i 0}^{\prime}, \overrightarrow{\boldsymbol{r}}^{\prime}\right)\right] \cdot \mathbf{G}\left(\vec{r}, \overrightarrow{\boldsymbol{r}}^{\prime}\right) \frac{1}{v_{T}\left(t_{i}^{\prime}, \overrightarrow{\boldsymbol{r}}^{\prime}\right)} d V^{\prime} \\
& -\frac{1}{P_{C}(t, \overrightarrow{\boldsymbol{r}}) \boldsymbol{v}_{\boldsymbol{r e l}}(t, \overrightarrow{\boldsymbol{r}})} \iiint_{\mathrm{V}} \frac{Z_{V}\left(t_{i 0}^{\prime}, \overrightarrow{\boldsymbol{r}}^{\prime}\right)}{v_{T}\left(t_{i 0}^{\prime}, \overrightarrow{\boldsymbol{r}}^{\prime}\right)} Q_{i 0} \boldsymbol{\nabla}\left[\frac{1}{4 \pi} \frac{1}{\left|\overrightarrow{\boldsymbol{r}}-\overrightarrow{\boldsymbol{r}}^{\prime}\right|^{2}}\right] \cdot \overrightarrow{\boldsymbol{u}}\left(t_{i 0}^{\prime}, \overrightarrow{\boldsymbol{r}}^{\prime}\right) d V^{\prime}
\end{aligned}
$$




$$
\begin{gathered}
+\iiint_{\mathrm{V}} Z_{V}\left(t_{i 0}^{\prime}, \overrightarrow{\boldsymbol{r}}^{\prime}\right) Q_{i 0}\left(t, t_{i 0}^{\prime}\right) \mathbf{G}\left(\overrightarrow{\boldsymbol{r}}, \overrightarrow{\boldsymbol{r}}^{\prime}\right) \cdot \overrightarrow{\boldsymbol{g}} \varphi_{i 0} \frac{1}{v_{T^{2}}\left(t_{i 0}^{\prime}, \overrightarrow{\boldsymbol{r}}^{\prime}\right)} d V^{\prime} \\
+\frac{\mathbf{1}}{P_{c}(t, \overrightarrow{\boldsymbol{r}}) v_{\boldsymbol{r e l}}(t, \overrightarrow{\boldsymbol{r}})} \iiint_{\mathrm{V}} Q_{i 0} \frac{d}{d t_{i 0}^{\prime}}\left[\varphi_{i 0} \frac{Z_{V}\left(t_{i 0}^{\prime}, \overrightarrow{\boldsymbol{r}}^{\prime}\right)}{v_{T}\left(t_{i 0}^{\prime}, \overrightarrow{\boldsymbol{r}}^{\prime}\right)} \overrightarrow{\boldsymbol{g}}\right] \cdot \mathbf{G}\left(\vec{r}, \overrightarrow{\boldsymbol{r}}^{\prime}\right) \frac{1}{v_{T}\left(t_{i}^{\prime}, \overrightarrow{\boldsymbol{r}}^{\prime}\right)} d V^{\prime} \\
\quad-\frac{\mathbf{1}}{P_{c}(t, \overrightarrow{\boldsymbol{r}}) v_{\text {rel }}(t, \overrightarrow{\boldsymbol{r}})} \iiint_{\mathrm{V}} Q_{i 0} Z_{V}\left(t_{i}^{\prime}, \overrightarrow{\boldsymbol{r}}^{\prime}\right) \mathbf{G}\left(\vec{r}, \overrightarrow{\boldsymbol{r}}^{\prime}\right) \cdot \overrightarrow{\boldsymbol{g}} \frac{1}{v_{T^{2}\left(t_{i 0}^{\prime}, \overrightarrow{\boldsymbol{r}}^{\prime}\right)}} d V^{\prime} \\
\quad-\frac{\mathbf{1}}{P_{c}(t, \overrightarrow{\boldsymbol{r}}) v_{\boldsymbol{r e l}}(t, \overrightarrow{\boldsymbol{r}})} \iiint_{\mathrm{V}} \frac{Z_{V}\left(t_{i 0}^{\prime}, \overrightarrow{\boldsymbol{r}}^{\prime}\right)}{v_{T}\left(t_{i 0}^{\prime}, \overrightarrow{\boldsymbol{r}}^{\prime}\right)} \varphi_{i 0} Q_{i 0} \nabla\left[\frac{1}{4 \pi} \frac{1}{\left|\overrightarrow{\boldsymbol{r}}-\overrightarrow{\boldsymbol{r}}^{\prime}\right|^{2}}\right] \cdot \overrightarrow{\boldsymbol{g}} d V^{\prime},
\end{gathered}
$$

where $Z_{V}(t, \overrightarrow{\boldsymbol{r}})$ the rate of collisions per unit volume in the given non-moving point $\overrightarrow{\boldsymbol{r}}$ at time $t$, which is expressed as

$$
Z_{V}(t, \overrightarrow{\boldsymbol{r}})=\frac{1}{2} n(t, \overrightarrow{\boldsymbol{r}}) P_{c}(t, \overrightarrow{\boldsymbol{r}}) \boldsymbol{v}_{\boldsymbol{r e l}}(t, \overrightarrow{\boldsymbol{r}}),
$$

The equation above defines $\overrightarrow{\boldsymbol{u}}(t, \overrightarrow{\boldsymbol{r}})$ as an implicit function of $\overrightarrow{\boldsymbol{r}}$ in space occupied by the model gas at a given time $t$. Here the mass balance equation is formed by considering a unique combination of converging ballistic particles from the entire model gas system and the diverging the same point at the same time $t$ ballistic particles. Besides, each of the converging ballistic particles can target point $\overrightarrow{\boldsymbol{r}}$ at given time $t$ and originates from a preceding collision within the model gas system at a time earlier than time $t$. Such a combination of converging and diverging ballistic particles capable of targeting or escaping point $\overrightarrow{\boldsymbol{r}}$ at given time $t$ is treated as an exhaustive combination. Therefore, from a physical viewpoint, the solution $\overrightarrow{\boldsymbol{u}}(t, \overrightarrow{\boldsymbol{r}})$ is unique.

Considering that point $\overrightarrow{\boldsymbol{r}}$ is not included in integration for converging ballistic particles, the first right-hand term of Equation (79) is zeroed. Comparing the second and the third right-hand terms of the equation above and considering the limitation of Equation (64), the third right-hand term can be neglected. Next, comparing the fourth and fifth right-hand terms of the equation above and considering the limitation of Equation (64), the fifth right-hand term can be neglected. Finally, comparing the sevenths and the eighths right-hand terms of the equation above and considering the limitation of Equation (64), the eighth right-hand term can be neglected. Also, comparing the sevenths and the ninths right-hand terms of the equation above, we may suggest that the ninths right-hand term can be neglected because of the low value of the coefficient $\frac{1}{P_{c} v_{r e l}}$.

Also, we need to estimate the sixths and the tenth right-hand terms of the equation above. First, we need to note these terms may have a significant value only within the point of singularity at $\overrightarrow{\boldsymbol{r}}^{\prime} \rightarrow \overrightarrow{\boldsymbol{r}}$ since the values of both $Q_{i 0}$ and $\frac{1}{\left|\overrightarrow{\boldsymbol{r}}-\overrightarrow{\boldsymbol{r}}^{\prime}\right|^{2}}$ functions are decreased with increasing $\left|\overrightarrow{\boldsymbol{r}}-\overrightarrow{\boldsymbol{r}}^{\prime}\right|$, where the product of others under integral functions are varied smoothly within the space occupied by the model gas. It implies the validity of these transformations of the integral in the sixths term, for certainty:

$$
\begin{gathered}
\iiint_{V} \frac{Z_{V}\left(t_{i 0}^{\prime}, \overrightarrow{\boldsymbol{r}}^{\prime}\right)}{v_{T}\left(t_{i 0}^{\prime}, \overrightarrow{\boldsymbol{r}}^{\prime}\right)} Q_{i 0} \nabla\left[\frac{1}{4 \pi} \frac{1}{\left|\overrightarrow{\boldsymbol{r}}-\overrightarrow{\boldsymbol{r}}^{\prime}\right|^{2}}\right] \cdot \overrightarrow{\boldsymbol{u}}\left(t_{i 0}^{\prime}, \overrightarrow{\boldsymbol{r}}^{\prime}\right) d V^{\prime} \\
\cong-\left[Q_{i 0}\left(t, t_{i 0}^{\prime}\right) \frac{Z_{V}\left(t_{i 0}^{\prime}, \overrightarrow{\boldsymbol{r}}^{\prime}\right)}{v_{T}\left(t_{i 0}^{\prime}, \overrightarrow{\boldsymbol{r}}^{\prime}\right)} \overrightarrow{\boldsymbol{u}}\left(t_{i 0}^{\prime}, \overrightarrow{\boldsymbol{r}}^{\prime}\right)\right]_{\overrightarrow{\boldsymbol{r}}^{\prime}=\overrightarrow{\boldsymbol{r}}} \cdot \iiint_{V^{\prime} \rightarrow 0} \nabla^{\prime}\left[\frac{1}{4 \pi} \frac{1}{\left|\overrightarrow{\boldsymbol{r}}-\overrightarrow{\boldsymbol{r}}^{\prime}\right|^{2}}\right] d V^{\prime}
\end{gathered}
$$




$$
\begin{gathered}
=+\left[Q_{i 0}\left(t, t_{i 0}^{\prime}\right) \frac{Z_{V}\left(t_{i 0}^{\prime}, \overrightarrow{\boldsymbol{r}}^{\prime}\right)}{v_{T}\left(t_{i 0}^{\prime}, \overrightarrow{\boldsymbol{r}}^{\prime}\right)} \overrightarrow{\boldsymbol{u}}\left(t_{i 0}^{\prime}, \overrightarrow{\boldsymbol{r}}^{\prime}\right)\right]_{\overrightarrow{\boldsymbol{r}}^{\prime}=\overrightarrow{\boldsymbol{r}}} \cdot \oiint_{A_{s}}\left[\frac{1}{4 \pi} \frac{1}{\left|\overrightarrow{\boldsymbol{r}}-\overrightarrow{\boldsymbol{r}}^{\prime}\right|^{2}}\right] \overrightarrow{\boldsymbol{n}}_{i 0} d A_{s} \\
\cong+\left[Q_{i 0}\left(t, t_{i 0}^{\prime}\right) \frac{Z_{V}\left(t_{i 0}^{\prime}, \overrightarrow{\boldsymbol{r}}^{\prime}\right)}{v_{T}\left(t_{i 0}^{\prime}, \overrightarrow{\boldsymbol{r}}^{\prime}\right)} \overrightarrow{\boldsymbol{u}}\left(t_{i 0}^{\prime}, \overrightarrow{\boldsymbol{r}}^{\prime}\right)\right]_{\overrightarrow{\boldsymbol{r}}^{\prime}=\overrightarrow{\boldsymbol{r}}} \cdot\left\{\frac{1}{4 \pi} \frac{1}{\left|\overrightarrow{\boldsymbol{r}}-\overrightarrow{\boldsymbol{r}}^{\prime}\right|^{2}} \overrightarrow{\boldsymbol{n}}_{\boldsymbol{i} 0} 4 \pi\left|\overrightarrow{\boldsymbol{r}}-\overrightarrow{\boldsymbol{r}}^{\prime}\right|^{2}\right\}_{\overrightarrow{\boldsymbol{r}}^{\prime}=\overrightarrow{\boldsymbol{r}}} \\
=\frac{Z_{V}(t, \overrightarrow{\boldsymbol{r}})}{v_{T}(t, \overrightarrow{\boldsymbol{r}})} \overrightarrow{\boldsymbol{u}}(t, \overrightarrow{\boldsymbol{r}}) \cdot\left\{\overrightarrow{\boldsymbol{n}}_{\boldsymbol{i} \mathbf{0}}\right\}_{\overrightarrow{\boldsymbol{r}}^{\prime}=\overrightarrow{\boldsymbol{r}}}=\overrightarrow{0} .
\end{gathered}
$$

Analogously, one can determine zero value for the integral in the tenth term of Equation (79). Because of the approximations described above, Equation (79) is reduced to:

$$
\begin{aligned}
& \frac{\partial}{\partial t} n(t, \overrightarrow{\boldsymbol{r}})+Z_{V}(t, \overrightarrow{\boldsymbol{r}})+\frac{1}{2} \boldsymbol{\nabla} \cdot[n(t, \vec{r}) \overrightarrow{\boldsymbol{u}}(t, \overrightarrow{\boldsymbol{r}})] \\
& =P_{c}(t, \overrightarrow{\boldsymbol{r}}) \boldsymbol{v}_{\boldsymbol{r e l}}(t, \overrightarrow{\boldsymbol{r}}) \iiint_{\mathrm{V}} Z_{V}\left(t_{i 0}^{\prime}, \overrightarrow{\boldsymbol{r}}^{\prime}\right) \frac{1}{v_{T}\left(t_{i 0}^{\prime}, \overrightarrow{\boldsymbol{r}}^{\prime}\right)} Q_{i 0}\left(t, t_{i 0}^{\prime}\right) \frac{1}{4 \pi} \frac{1}{\left|\overrightarrow{\boldsymbol{r}}-\overrightarrow{\boldsymbol{r}}^{\prime}\right|^{2}} d V^{\prime} \\
& +P_{c}(t, \overrightarrow{\boldsymbol{r}}) \boldsymbol{v}_{\boldsymbol{r e l}}(t, \overrightarrow{\boldsymbol{r}}) \iiint_{\mathrm{V}} Z_{V}\left(t_{i 0}^{\prime}, \overrightarrow{\boldsymbol{r}}^{\prime}\right) \frac{1}{v_{T^{2}\left(t_{i 0}^{\prime}, \overrightarrow{\boldsymbol{r}}^{\prime}\right)}} Q_{i 0}\left(t, t_{i 0}^{\prime}\right) \mathbf{G}\left(\overrightarrow{\boldsymbol{r}}, \overrightarrow{\boldsymbol{r}}^{\prime}\right) \cdot \overrightarrow{\boldsymbol{u}}\left(t_{i 0}^{\prime}, \overrightarrow{\boldsymbol{r}}^{\prime}\right) d V^{\prime} \\
& +P_{c}(t, \overrightarrow{\boldsymbol{r}}) \boldsymbol{v}_{\boldsymbol{r e l}}(t, \overrightarrow{\boldsymbol{r}}) \iiint_{\mathrm{V}} Z_{V}\left(t_{i 0}^{\prime}, \overrightarrow{\boldsymbol{r}}^{\prime}\right) \frac{1}{v_{T^{2}\left(t_{i 0}^{\prime}, \vec{r}^{\prime}\right)}} Q_{i 0}\left(t, t_{i 0}^{\prime}\right) \mathbf{G}\left(\overrightarrow{\boldsymbol{r}}, \overrightarrow{\boldsymbol{r}}^{\prime}\right) \cdot \overrightarrow{\boldsymbol{g}} \varphi_{i 0} d V^{\prime} .
\end{aligned}
$$

Here point $\overrightarrow{\boldsymbol{r}}$ is not included in integration for converging ballistic particles. It implies that Equation (82) defines $\overrightarrow{\boldsymbol{u}}$ at $t \geq 0$ as an implicit function of $\overrightarrow{\boldsymbol{r}}$ on $\mathbb{R}^{\mathbf{3}}$, i.e. $\overrightarrow{\boldsymbol{r}} \in \mathbb{R}^{\mathbf{3}}$.

\subsection{Analytical representation of an integro-differential form of the momentum balance equation in the collision-dominated flow regime}

Here we again should note that the momentum balance equation is formed by considering a unique combination of ballistic particles converging from the entire model gas system in a given point at a given time and the diverging ballistic particles from the given point at the given time. Besides, each of the converging ballistic particles can target point $\overrightarrow{\boldsymbol{r}}$ at given time $t$ and originates from a preceding collision within the model gas system at a time earlier than time $t$. Such a combination of converging and diverging ballistic particles capable of targeting or escaping point $\overrightarrow{\boldsymbol{r}}$ at given time $t$ is treated as an exhaustive combination. Therefore, from a physical viewpoint, the solution $\overrightarrow{\boldsymbol{u}}(t, \overrightarrow{\boldsymbol{r}})$ is unique.

To formulate an integro-differential form of momentum balance equation in a given nonmoving point of space occupied by the model at a given time, we will modify Equation (56) as follows:

(1) Assigning

$$
\overrightarrow{\boldsymbol{v}}_{+}\left(t, \overrightarrow{\boldsymbol{r}}, t_{a}^{\prime}, \overrightarrow{\boldsymbol{r}}^{\prime}\right) \cong v_{\mathrm{T}}(t, \overrightarrow{\boldsymbol{r}}) \overrightarrow{\boldsymbol{n}}_{+\mathbf{0}}+\overrightarrow{\boldsymbol{u}}(t, \overrightarrow{\boldsymbol{r}})+\overrightarrow{\boldsymbol{g}} \varphi_{+0}
$$

and

$$
\overrightarrow{\boldsymbol{v}}\left(t_{i}^{\prime}, \overrightarrow{\boldsymbol{r}}^{\prime}, t, \overrightarrow{\boldsymbol{r}}\right) \cong v_{T}\left(t_{i 0}^{\prime}, \overrightarrow{\boldsymbol{r}}^{\prime}\right) \overrightarrow{\boldsymbol{n}}_{\boldsymbol{i} \mathbf{0}}+\overrightarrow{\boldsymbol{u}}\left(t_{i 0}^{\prime}, \overrightarrow{\boldsymbol{r}}^{\prime}\right)+\overrightarrow{\boldsymbol{g}} \varphi_{i 0}
$$

(2) Substituting approximations for functions involving in Equation (56) by Equations (83), (84), and from Equation (65) to Equation (78) and executing vector differentiation. 


\section{(c) $(1)(9)$}

(3) Executing limit $\overrightarrow{\boldsymbol{r}}^{\prime} \rightarrow \overrightarrow{\boldsymbol{r}}$ in the second left-hand term of the resulting approximated integro-differential momentum balance equation. We obtain:

$$
\begin{aligned}
& \frac{1}{P_{c} \boldsymbol{v}_{\text {rel }}}\left\{\frac{\partial}{\partial t}[n \overrightarrow{\boldsymbol{u}}]+2 Z_{V} \overrightarrow{\boldsymbol{u}}+\frac{1}{2} \boldsymbol{\nabla}\left(n{v_{T}}^{2}\right)+\frac{1}{2} \boldsymbol{\nabla} \cdot[n \overrightarrow{\boldsymbol{u}} \overrightarrow{\boldsymbol{u}}]-2 n \overrightarrow{\boldsymbol{g}}+\frac{1}{m} \boldsymbol{\nabla} p\right\} \\
& \cong \iiint_{V} Z_{V} \frac{1}{v_{T}} Q_{i 0} \mathbf{G} \cdot\left[\overrightarrow{\boldsymbol{n}}_{\boldsymbol{i 0}} \overrightarrow{\boldsymbol{n}}_{\boldsymbol{i} \mathbf{0}} v_{T}+\left(\overrightarrow{\boldsymbol{n}}_{\boldsymbol{i} \mathbf{0}} \overrightarrow{\boldsymbol{u}}+\overrightarrow{\boldsymbol{u}} \overrightarrow{\boldsymbol{n}}_{\boldsymbol{i 0}}\right)+\varphi_{i 0}\left(\overrightarrow{\boldsymbol{n}}_{\boldsymbol{i 0}} \overrightarrow{\boldsymbol{g}}+\overrightarrow{\boldsymbol{g}}_{\boldsymbol{i} \mathbf{0}}\right)\right] d V^{\prime} \\
& -\frac{\mathbf{1}}{P_{c} \boldsymbol{v}_{\text {rel }}} \iiint_{\mathrm{V}} Q_{i 0} \boldsymbol{\nabla} \cdot\left\{Z_{V}\left[\mathbf{G} \overrightarrow{\boldsymbol{n}}_{\boldsymbol{i 0}} v_{T}+(\mathbf{G} \overrightarrow{\boldsymbol{u}}+\overrightarrow{\boldsymbol{u}} \mathbf{G})+\varphi_{i 0}(\mathbf{G} \overrightarrow{\boldsymbol{g}}+\overrightarrow{\boldsymbol{g}} \mathbf{G})\right]\right\} d V^{\prime} \\
& +\iiint_{V} Z_{V} \frac{1}{v_{T}{ }^{2}} Q_{i 0} \mathbf{G} \cdot\left[\overrightarrow{\boldsymbol{u}} \overrightarrow{\boldsymbol{u}}+\varphi_{i 0}(\overrightarrow{\boldsymbol{u}} \vec{g}+\overrightarrow{\boldsymbol{g}} \overrightarrow{\boldsymbol{u}})+\overrightarrow{\boldsymbol{g}} \overrightarrow{\boldsymbol{g}} \varphi_{i 0}^{2}\right] d V^{\prime} \\
& -\frac{1}{P_{c} v_{r e l}} \iiint_{V} Q_{i 0} \boldsymbol{\nabla} \cdot\left\{Z_{V} \frac{1}{v_{T}} \frac{1}{4 \pi} \frac{1}{\left|\overrightarrow{\boldsymbol{r}}-\overrightarrow{\boldsymbol{r}}^{\prime}\right|^{2}}\left[\overrightarrow{\boldsymbol{u} \boldsymbol{u}}+\varphi_{i 0}(\overrightarrow{\boldsymbol{u}} \overrightarrow{\boldsymbol{g}}+\overrightarrow{\boldsymbol{g}} \overrightarrow{\boldsymbol{u}})+\overrightarrow{\boldsymbol{g}} \overrightarrow{\boldsymbol{g}} \varphi_{i 0}^{2}\right]\right\} d V^{\prime} .
\end{aligned}
$$

As it has been commented above regarding Equation (79), Equation (85) defines $\overrightarrow{\boldsymbol{u}}(t, \overrightarrow{\boldsymbol{r}})$ as an implicit function of $\overrightarrow{\boldsymbol{r}}$ in space occupied by the model gas at a given time $t$.

(4) Executing a procedure of neglecting the insignificant terms by following the procedures described in the previous sub-section. Here, in the interest of simplicity, we take into the account the approximation expressed by Equation (63), which allows us to neglect the second and the fourth right-hand terms in the equation above. Remembering that point $\overrightarrow{\boldsymbol{r}}$ is not included in integration for converging ballistic particles, we obtain the following integro-differential form of the momentum balance equation:

$$
\begin{array}{r}
\frac{\partial}{\partial t}[n \overrightarrow{\boldsymbol{u}}]+2 Z_{V} \overrightarrow{\boldsymbol{u}}+\frac{1}{2} \boldsymbol{\nabla}\left(n v_{T}^{2}\right)+\frac{1}{2} \boldsymbol{\nabla} \cdot[n \overrightarrow{\boldsymbol{u}} \overrightarrow{\boldsymbol{u}}]-2 n \overrightarrow{\boldsymbol{g}}+\frac{1}{m} \boldsymbol{\nabla} p \\
\cong P_{c} \boldsymbol{v}_{\boldsymbol{r e l}} \iiint_{\mathrm{V}} Z_{V} Q_{i 0} \mathbf{G} d V^{\prime} \\
+2 P_{c} \boldsymbol{v}_{\text {rel }} \iiint_{\mathrm{V}} Z_{V} \frac{1}{v_{T}} Q_{i 0} \frac{1}{4 \pi} \frac{1}{\left|\overrightarrow{\boldsymbol{r}}-\overrightarrow{\boldsymbol{r}}^{\prime}\right|^{2}}[\overrightarrow{\boldsymbol{u}}] d V^{\prime} \\
+2 P_{c} \boldsymbol{v}_{\text {rel }} \iiint_{\mathrm{V}} Z_{V} \frac{1}{v_{T}} Q_{i 0} \frac{1}{4 \pi} \frac{1}{\left|\overrightarrow{\boldsymbol{r}}-\overrightarrow{\boldsymbol{r}}^{\prime}\right|^{2}} \varphi_{i 0} \overrightarrow{\boldsymbol{g}} d V^{\prime} \\
+P_{c} \boldsymbol{v}_{\text {rel }} \iiint_{\mathrm{V}} Z_{V} \frac{1}{v_{T^{2}}} Q_{i 0}(\mathbf{G} \cdot \overrightarrow{\boldsymbol{u}}) \overrightarrow{\boldsymbol{u}} d V^{\prime} \\
+P_{c} \boldsymbol{v}_{\text {rel }} \iiint_{\mathrm{V}} Z_{V} \frac{1}{v_{T}^{2}} Q_{i 0} \mathbf{G} \cdot\left[\varphi_{i 0}\left[(\overrightarrow{\boldsymbol{u}} \boldsymbol{g}+\overrightarrow{\boldsymbol{g}} \overrightarrow{\boldsymbol{u}})+\overrightarrow{\boldsymbol{g}} \overrightarrow{\boldsymbol{g}} \varphi_{i 0}\right]\right] d V^{\prime}
\end{array}
$$

It implies that Equation (86) defines $\overrightarrow{\boldsymbol{u}}$ at $t \geq 0$ as an implicit function of $\overrightarrow{\boldsymbol{r}}$ on $\mathbb{R}^{\mathbf{3}}$, i.e. $\overrightarrow{\boldsymbol{r}} \in \mathbb{R}^{\mathbf{3}}$.

\section{Reducing Integro-Differential Forms of Mass and Momentum Balance Equations into Corresponding Differential Forms}

According to Tenenbaum and Pollard [14], to test whether an implicit function defined by the relation $f(x, y)=0$ is a solution of a given differential equation; there is a need to show that the function does satisfy a given differential equation on an interval I: $a<x<b$. Then the relation $f(x, y)=0$ is called an implicit solution of the differential equation.

Definition 3.6 [14]: A relation $f(x, y)=0$ will be called an implicit solution of the differential equation

$$
F\left(x, y, y^{\prime}, \cdots y^{(n)}\right)=0
$$


on an interval I: $a<x<b$, if

1. it defines $y$ as an implicit function of $x$ on $I$, i.e., if there exists a function $g(x)$ defined on $\boldsymbol{I}$ such that $f(x, g(x))=0$ for every $x$ on $\boldsymbol{I}$, and if

2. $g(x)$ satisfies (87), i.e., if

$$
F\left(x, g(x), g(x)^{\prime}, \cdots g(x)^{(n)}\right)=0
$$

for every $x$ on $\boldsymbol{I}$.

The standard procedure in calculus to prove that an implicit function defined by relationship $f(x, y)=0$ (a) is a solution of a given differential equation $F\left(x, y, y^{\prime}, \cdots y^{(n)}\right)=0$ on an interval $I: a<x<b$ (b) is the following [14]: "Differentiate (a) implicitly. If it yields (b), then (a) is said to be an implicit solution of (b)," if $f(x, y)$ defines $y$ as an implicit function of $x$ on $I$.

To reduce an equation to an ordinary differential equation, we apply the method of differentiation for integral equations (ones, twice, and so on) with subsequent elimination of the terms belonging to the original equation [15]. Specifically, we will use this technique for reducing vector integro-differential balance equations into corresponding vector differential balance equations. Here we need also note that the point $\overrightarrow{\boldsymbol{r}}$ should be included in integration since we have to apply the operator of vector differentiation in this point. In the domain of integration $\Omega$ in which $\overrightarrow{\boldsymbol{r}}^{\prime} \neq \overrightarrow{\boldsymbol{r}}$, operation of differentiation regarding a parameter $\overrightarrow{\boldsymbol{r}}$ is interchangeable with the operation of the integration over some other variable $\overrightarrow{\boldsymbol{r}}^{\prime}$. Even if domain $\Gamma$ includes singularity point $\overrightarrow{\boldsymbol{r}}$, the integrals containing the term $\boldsymbol{\nabla}\left[\frac{1}{4 \pi} \frac{1}{\left|\overrightarrow{\boldsymbol{r}}-\overrightarrow{\boldsymbol{r}}^{\prime}\right|^{2}}\right]$ are zeroed, as it was shown as an example by Equation (81). The "fate" of other integrals containing singularity point $\overrightarrow{\boldsymbol{r}}$ will be examined afterward.

\subsection{Reducing the integro-differential form of mass balance equation into a corresponding vector differential form}

Deriving a vector differential form of mass balance equation is shown:

Step 1. Normalizing Equation (82) by $P_{c} \boldsymbol{v}_{\text {rel }}$, then applying differential operator $\nabla$ to the left- and right-hands of the equation and executing procedures of neglecting insignificant terms, which are similar to the described in Section III. This leads to establishing the following equality:

$$
\begin{gathered}
\frac{\mathbf{1}}{P_{c} \boldsymbol{v}_{r e l}} \nabla\left[\frac{\mathbf{1}}{P_{c} \boldsymbol{v}_{r e l}}\left\{\frac{\partial}{\partial t} n+Z_{V}+\frac{1}{2} \nabla \cdot[n \overrightarrow{\boldsymbol{u}}]\right\}\right] \\
\cong-\iiint_{\mathrm{V}} \frac{Z_{V}\left(t_{i 0}^{\prime}, \overrightarrow{\boldsymbol{r}}^{\prime}\right)}{v_{T}\left(t_{i 0}^{\prime}, \overrightarrow{\boldsymbol{r}}^{\prime}\right)} \frac{1}{v_{T}\left(t_{i 0}^{\prime}, \overrightarrow{\boldsymbol{r}}^{\prime}\right)} Q_{i 0}\left(t, t_{i 0}^{\prime}\right) \mathbf{G}\left(\overrightarrow{\boldsymbol{r}}, \overrightarrow{\boldsymbol{r}}^{\prime}\right) d V^{\prime} \\
-\iiint_{\mathrm{V}} \frac{Z_{V}\left(t_{i 0}^{\prime}, \overrightarrow{\boldsymbol{r}}^{\prime}\right)}{v_{T}\left(t_{i 0}^{\prime}, \overrightarrow{\boldsymbol{r}}^{\prime}\right)} \frac{1}{v_{T^{2}}\left(t_{i 0}^{\prime}, \overrightarrow{\boldsymbol{r}}^{\prime}\right)} Q_{i 0}\left(t, t_{i 0}^{\prime}\right) \frac{1}{4 \pi} \frac{1}{\left|\overrightarrow{\boldsymbol{r}}-\overrightarrow{\boldsymbol{r}}^{\prime}\right|^{2}} \overrightarrow{\boldsymbol{u}}\left(t_{i 0}^{\prime}, \overrightarrow{\boldsymbol{r}}^{\prime}\right) d V^{\prime} \\
-\iiint_{\mathrm{V}} \frac{Z_{V}\left(t_{i 0}^{\prime}, \overrightarrow{\boldsymbol{r}}^{\prime}\right)}{v_{T}\left(t_{i 0}^{\prime}, \overrightarrow{\boldsymbol{r}}^{\prime}\right)} \frac{1}{v_{T}^{2}\left(t_{i 0}^{\prime}, \overrightarrow{\boldsymbol{r}}^{\prime}\right)} Q_{i 0}\left(t, t_{i 0}^{\prime}\right) \frac{1}{4 \pi} \frac{1}{\left|\overrightarrow{\boldsymbol{r}}-\overrightarrow{\boldsymbol{r}}^{\prime}\right|^{2}} \overrightarrow{\boldsymbol{g}} \varphi_{i 0} d V^{\prime} \\
+\frac{\mathbf{1}}{P_{c} \boldsymbol{v}_{r e l}} \iiint_{\mathrm{V}} \frac{Z_{V}\left(t_{i 0}^{\prime}, \overrightarrow{\boldsymbol{r}}^{\prime}\right)}{v_{T^{2}}\left(t_{i 0}^{\prime}, \overrightarrow{\boldsymbol{r}}^{\prime}\right)} Q_{i 0}\left(t, t_{i 0}^{\prime}\right)(\overrightarrow{\boldsymbol{u}} \cdot \boldsymbol{\nabla}) \boldsymbol{G} d V^{\prime}
\end{gathered}
$$




$$
+\frac{\mathbf{1}}{P_{c} \boldsymbol{v}_{\text {rel }}} \iiint_{\mathrm{V}} \frac{Z_{V}\left(t_{i 0}^{\prime}, \overrightarrow{\boldsymbol{r}}^{\prime}\right)}{v_{T}\left(t_{i 0}^{\prime}, \overrightarrow{\boldsymbol{r}}^{\prime}\right)} Q_{i 0} \frac{1}{v_{T}^{2}\left(t_{i 0}^{\prime}, \vec{r}^{\prime}\right)} \frac{1}{4 \pi} \frac{1}{\left|\overrightarrow{\boldsymbol{r}}-\overrightarrow{\boldsymbol{r}}^{\prime}\right|^{2}} \overrightarrow{\boldsymbol{g}} d V^{\prime} .
$$

Step 2. Applying divergence operator $\nabla \cdot$ to the left- and right-hands of the equation above and neglecting insignificant terms by the steps, which are similar to the described in Section III:

$$
\begin{aligned}
& \boldsymbol{\nabla} \cdot\left\{\frac{\mathbf{1}}{P_{c} \boldsymbol{v}_{\text {rel }}} \nabla\left[\frac{\mathbf{1}}{P_{c} \boldsymbol{v}_{\text {rel }}}\left\{\frac{\partial}{\partial t} n+Z_{V}+\frac{1}{2} \boldsymbol{\nabla} \cdot[n \overrightarrow{\boldsymbol{u}}]\right\}\right]\right\} \\
& \cong+P_{c} \boldsymbol{v}_{\text {rel }} \iiint_{\mathrm{V}} \frac{Z_{V}\left(t_{i 0}^{\prime}, \vec{r}^{\prime}\right)}{v_{T}^{2}\left(t_{i 0}^{\prime}, \overrightarrow{\boldsymbol{r}}^{\prime}\right)} \frac{1}{v_{T}\left(t_{i 0}^{\prime}, \vec{r}^{\prime}\right)} Q_{i 0} \frac{1}{4 \pi} \frac{1}{\left|\overrightarrow{\boldsymbol{r}}-\overrightarrow{\boldsymbol{r}}^{\prime}\right|^{2}} d V^{\prime} \\
& +P_{c} \boldsymbol{v}_{\text {rel }} \iiint_{\mathrm{V}} \frac{Z_{V}\left(t_{i 0}^{\prime}, \overrightarrow{\boldsymbol{r}}^{\prime}\right)}{v_{T^{2}}{ }^{\prime}\left(t_{i 0}^{\prime}, \overrightarrow{\boldsymbol{r}}^{\prime}\right)} \frac{1}{v_{T}^{2}\left(t_{i 0}^{\prime}, \overrightarrow{\boldsymbol{r}}^{\prime}\right)} Q_{i 0} \mathbf{G}\left(\overrightarrow{\boldsymbol{r}}, \overrightarrow{\boldsymbol{r}}^{\prime}\right) \cdot \overrightarrow{\boldsymbol{u}}\left(t_{i 0}^{\prime}, \overrightarrow{\boldsymbol{r}}^{\prime}\right) d V^{\prime} \\
& +P_{C} \boldsymbol{v}_{\boldsymbol{r e l}} \iiint_{\mathrm{V}} \frac{Z_{V}\left(t_{i 0}^{\prime}, \overrightarrow{\boldsymbol{r}}^{\prime}\right)}{v_{T}^{2}\left(t_{i 0}^{\prime}, \overrightarrow{\boldsymbol{r}}^{\prime}\right)} \frac{1}{v_{T^{2}}\left(t_{i 0}^{\prime}, \overrightarrow{\boldsymbol{r}}^{\prime}\right)} Q_{i 0} \mathbf{G}\left(\overrightarrow{\boldsymbol{r}}, \overrightarrow{\boldsymbol{r}}^{\prime}\right) \cdot \overrightarrow{\boldsymbol{g}} \varphi_{i 0} d V^{\prime} \\
& -\frac{Z_{V}}{v_{T}^{2}}+\frac{1}{P_{c} v_{r e l}} \nabla \cdot\left(\overrightarrow{\boldsymbol{u}} \frac{Z_{V}}{v_{T}^{2}}\right) .
\end{aligned}
$$

Step 3. Comparing the first four right-hand terms in the equation above and Equation (82), we may suggest a modification of Equation (82) by introducing a coefficient of proportionality to the mass, $\frac{1}{v_{T}^{2}}$. This results in obtaining of the following equation:

$$
\begin{gathered}
\frac{\partial}{\partial t}\left[\frac{n}{v_{T}^{2}}\right]+\frac{Z_{V}}{v_{T}^{2}}+\frac{1}{2} \boldsymbol{\nabla} \cdot\left[\overrightarrow{\boldsymbol{u}} \frac{n}{v_{T}^{2}}\right] \\
\cong+P_{C} \boldsymbol{v}_{\text {rel }} \iiint_{\mathrm{V}} \frac{Z_{V}\left(t_{i 0}^{\prime}, \overrightarrow{\boldsymbol{r}}^{\prime}\right)}{v_{T}^{2}\left(t_{i 0}^{\prime}, \overrightarrow{\boldsymbol{r}}^{\prime}\right)} \frac{1}{v_{T}\left(t_{i 0}^{\prime}, \overrightarrow{\boldsymbol{r}}^{\prime}\right)} Q_{i 0} \frac{1}{4 \pi} \frac{1}{\left|\overrightarrow{\boldsymbol{r}}-\overrightarrow{\boldsymbol{r}}^{\prime}\right|^{2}} d V^{\prime} \\
+P_{C} \boldsymbol{v}_{\boldsymbol{r e l}} \iiint_{\mathrm{V}} \frac{Z_{V}\left(t_{i 0}^{\prime}, \overrightarrow{\boldsymbol{r}}^{\prime}\right)}{v_{T}^{2}\left(t_{i 0}^{\prime}, \overrightarrow{\boldsymbol{r}}^{\prime}\right)} \frac{1}{v_{T}^{2}\left(t_{i 0}^{\prime}, \overrightarrow{\boldsymbol{r}}^{\prime}\right)} Q_{i 0} \mathbf{G}\left(\overrightarrow{\boldsymbol{r}}, \overrightarrow{\boldsymbol{r}}^{\prime}\right) \cdot \overrightarrow{\boldsymbol{u}}\left(t_{i 0}^{\prime}, \overrightarrow{\boldsymbol{r}}^{\prime}\right) d V^{\prime} \\
+P_{C} \boldsymbol{v}_{\boldsymbol{r e l}} \iiint_{\mathrm{V}} \frac{Z_{V}\left(t_{i 0}^{\prime}, \overrightarrow{\boldsymbol{r}}^{\prime}\right)}{v_{T}^{2}\left(t_{i 0}^{\prime}, \overrightarrow{\boldsymbol{r}}^{\prime}\right)} \frac{1}{v_{T^{2}}\left(t_{i 0}^{\prime}, \overrightarrow{\boldsymbol{r}}^{\prime}\right)} Q_{i 0} \mathbf{G}\left(\overrightarrow{\boldsymbol{r}}, \overrightarrow{\boldsymbol{r}}^{\prime}\right) \cdot \overrightarrow{\boldsymbol{g}} \varphi_{i 0} d V^{\prime} .
\end{gathered}
$$

Substituting the first three right-hand terms of Equation (90) by the left-hand terms of Equ

$$
\frac{\partial}{\partial t}\left(\frac{n}{v_{T}^{2}}\right)+\boldsymbol{\nabla} \cdot\left(\overrightarrow{\boldsymbol{u}} \frac{n}{v_{T}^{2}}\right)=\frac{9}{16} \boldsymbol{\nabla} \cdot\left\{\frac{1}{P_{c} v_{T}} \nabla\left[\frac{1}{P_{c} v_{T}}\left\{\frac{\partial}{\partial t} n+\frac{2}{3} n P_{c} v_{T}+\frac{1}{2} \boldsymbol{\nabla} \cdot[n \overrightarrow{\boldsymbol{u}}]\right\}\right]\right\}-\frac{1}{2} \frac{1}{v_{T}^{2}} n \overrightarrow{\boldsymbol{u}} \cdot \frac{\boldsymbol{\nabla}\left(n v_{T}\right)}{n v_{T}}
$$

The equation above suggests that when considering the mass balance, the thermal velocity of particles should be considered. This is reasonable for gases composing the plurality of randomly moving particles. For an incompressible model gas at the uniform temperature and a lack of the acceleration field, Equation (92) is reduced to:

$$
\boldsymbol{\nabla} \cdot \overrightarrow{\boldsymbol{u}}=\frac{9}{32} \frac{1}{P_{c}^{2}} \boldsymbol{\nabla} \cdot \nabla(\boldsymbol{\nabla} \cdot \overrightarrow{\boldsymbol{u}})
$$

which is obtained by considering that $P_{c}, v_{r e l}$, and $v_{T}$ are constants. For high particle density, the right-hand of the equation above is limited to zero, so the equation above is approximated to the well-known continuity equation for incompressible fluid:

$$
\boldsymbol{\nabla} \cdot \overrightarrow{\boldsymbol{u}} \cong 0
$$

Since the vector differentiation of Equation (82) yields Equation (4) and Equation (82) defines $\overrightarrow{\boldsymbol{u}}$ at $t \geq 0$ as an implicit function of $\overrightarrow{\boldsymbol{r}}$ on $\mathbb{R}^{3}$, then Equation (82) is an implicit solution of Equation (4) in the region $\overrightarrow{\boldsymbol{r}} \in \mathbb{R}^{3}$ occupied by the model gas. 


\section{(c) $)(1) \Theta(\Theta)$}

4.2 Reducing the integro-differential form of momentum balance equation into a corresponding vector differential form

Deriving a vector differential form of mass balance equation is shown:

Step 1. Normalizing Equation (86) by $P_{c} \boldsymbol{v}_{\text {rel }}$, applying the differential operator $\nabla$ to the leftand right-hands of the equation, and executing procedures of neglecting insignificant terms, which are similar to those described in Section 3. This leads to establishing the following equality:

$$
\begin{aligned}
& \frac{\mathbf{1}}{P_{c} \boldsymbol{v}_{\text {rel }}} \nabla\left\{\frac{\mathbf{1}}{P_{c} \boldsymbol{v}_{\text {rel }}}\left[\frac{\partial}{\partial t}[n \overrightarrow{\boldsymbol{u}}]+2 Z_{V} \overrightarrow{\boldsymbol{u}}+\frac{1}{2} \boldsymbol{\nabla}\left(n v_{T}^{2}\right)+\frac{1}{2} \boldsymbol{\nabla} \cdot[n \overrightarrow{\boldsymbol{u}} \overrightarrow{\boldsymbol{u}}]-2 n \overrightarrow{\boldsymbol{g}}+\frac{1}{m} \boldsymbol{\nabla} p\right]\right\} \\
& =-\iiint_{\mathrm{V}} Z_{V} \frac{1}{v_{T}} Q_{i 0} \overrightarrow{\boldsymbol{n}}_{\boldsymbol{i} \mathbf{0}} \mathbf{G} d V^{\prime}+\frac{\mathbf{1}}{P_{c} \boldsymbol{v}_{\boldsymbol{r} e l}} \iiint_{\mathrm{V}} Z_{V} Q_{i 0} \nabla \mathbf{G} d V^{\prime}-2 \iiint_{\mathrm{V}} Z_{V} \frac{1}{v_{T}^{2}} Q_{i 0} \mathbf{G} \overrightarrow{\boldsymbol{u}} d V^{\prime} \\
& -2 \iiint_{\mathrm{V}} Z_{V} \frac{1}{v_{T}^{2}} Q_{i 0} \mathbf{G} \varphi_{i 0} \overrightarrow{\boldsymbol{g}} d V^{\prime}+2 \frac{\mathbf{1}}{P_{c} \boldsymbol{v}_{\boldsymbol{r e l}}} \iiint_{\mathrm{V}} Z_{V} \frac{1}{v_{T}^{2}} Q_{i 0} \mathbf{G} \overrightarrow{\boldsymbol{g}} d V^{\prime} m \\
& -\iiint_{\mathrm{V}} Z_{V} \frac{1}{v_{T}{ }^{3}} Q_{i 0} \overrightarrow{\boldsymbol{n}}_{\boldsymbol{i} \mathbf{0}}(\mathbf{G} \cdot \overrightarrow{\boldsymbol{u}}) \overrightarrow{\boldsymbol{u}} d V^{\prime}+\frac{1}{P_{c} \boldsymbol{v}_{r e l}} \iiint_{\mathrm{V}} Z_{V} \frac{1}{v_{T}^{2}} Q_{i 0}(\overrightarrow{\boldsymbol{u}} \cdot \nabla) \boldsymbol{G} \overrightarrow{\boldsymbol{u}} d V^{\prime} \\
& -\iiint_{V} Z_{V} \frac{1}{v_{T}^{3}} Q_{i 0} \overrightarrow{\boldsymbol{n}}_{\boldsymbol{i} \mathbf{0}} \mathbf{G} \cdot\left[\varphi_{i 0}\left[(\overrightarrow{\boldsymbol{u}} \boldsymbol{g}+\overrightarrow{\boldsymbol{g}} \overrightarrow{\boldsymbol{u}})+\overrightarrow{\boldsymbol{g}} \overrightarrow{\boldsymbol{g}} \varphi_{i 0}\right]\right] d V^{\prime} \\
& +\frac{1}{P_{c} v_{r e l}} \iiint_{V} Z_{V} \frac{1}{v_{T^{2}}} Q_{i 0}\left(\varphi_{i 0}\left[(\overrightarrow{\boldsymbol{u}} \vec{g}+\overrightarrow{\boldsymbol{g}} \overrightarrow{\boldsymbol{u}})+\overrightarrow{\boldsymbol{g}} \overrightarrow{\boldsymbol{g}} \varphi_{i 0}\right] \cdot \nabla\right) \mathbf{G} d V^{\prime}
\end{aligned}
$$

Step 2. Applying divergence operator $\nabla \cdot$ to the left- and right-hands of the equation above and neglecting insignificant terms by the procedures, which is similar to the described in Section III:

$$
\begin{aligned}
& \nabla \cdot\left\{\frac{\mathbf{1}}{P_{c} \boldsymbol{v}_{r e l}} \nabla\left\{\frac{\mathbf{1}}{P_{c} \boldsymbol{v}_{\text {rel }}}\left[\frac{\partial}{\partial t}[n \overrightarrow{\boldsymbol{u}}]+2 Z_{V} \overrightarrow{\boldsymbol{u}}+\frac{1}{2} \boldsymbol{\nabla}\left(n v_{T}^{2}\right)+\frac{1}{2} \boldsymbol{\nabla} \cdot[n \overrightarrow{\boldsymbol{u}} \overrightarrow{\boldsymbol{u}}]-2 n \overrightarrow{\boldsymbol{g}}+\frac{1}{m} \boldsymbol{\nabla} p\right]\right\}\right\} \\
& =P_{c} \boldsymbol{v}_{\text {rel }} \iiint_{\mathrm{V}} Z_{V} \frac{1}{v_{T^{2}}} Q_{i 0} \mathbf{G} d V^{\prime}+2 P_{c} \boldsymbol{v}_{\text {rel }} \iiint_{\mathrm{V}} Z_{V} \frac{1}{v_{T^{2}}} \frac{1}{v_{T}} Q_{i 0} \overrightarrow{\boldsymbol{u}} d V^{\prime} \\
& +2 P_{c} \boldsymbol{v}_{\text {rel }} \iiint_{\mathrm{V}} Z_{V} \frac{1}{v_{T}^{2}} \frac{1}{v_{T}} Q_{i 0} \frac{1}{4 \pi} \frac{1}{\left|\overrightarrow{\boldsymbol{r}}-\overrightarrow{\boldsymbol{r}}^{\prime}\right|^{2}} \varphi_{i 0} \overrightarrow{\boldsymbol{g}} d V^{\prime}+P_{c} \boldsymbol{v}_{\text {rel }} \iiint_{\mathrm{V}} Z_{V} \frac{1}{v_{T}^{2}} \frac{1}{v_{T}^{2}} Q_{i 0}(\mathbf{G} \cdot \overrightarrow{\boldsymbol{u}}) \overrightarrow{\boldsymbol{u}} d V^{\prime} \\
& +P_{c} \boldsymbol{v}_{\text {rel }} \iiint_{\mathrm{V}} Z_{V} \frac{1}{v_{T}^{2}} \frac{1}{v_{T}^{2}} Q_{i 0} \mathbf{G} \cdot\left[\varphi_{i 0}\left[(\overrightarrow{\boldsymbol{u}} \overrightarrow{\boldsymbol{g}}+\overrightarrow{\boldsymbol{g}} \overrightarrow{\boldsymbol{u}})+\overrightarrow{\boldsymbol{g}} \overrightarrow{\boldsymbol{g}} \varphi_{i 0}\right]\right] d V^{\prime} \\
& +\frac{1}{2} \frac{\boldsymbol{\nabla}\left(P_{c} \boldsymbol{v}_{\text {rel }}\right)}{P_{c} \boldsymbol{v}_{\text {rel }}} n+\frac{1}{2} \boldsymbol{\nabla} n+\frac{n}{v_{T^{2}}{ }^{2}} \overrightarrow{\boldsymbol{g}}-2 \frac{Z_{V}}{v_{T^{2}}{ }^{2}} \overrightarrow{\boldsymbol{u}}+\frac{1}{2} \frac{\boldsymbol{\nabla}\left(P_{c} \boldsymbol{v}_{\text {rel }}\right)}{P_{c} \boldsymbol{v}_{\text {rel }}} \cdot\left(\frac{n}{v_{T^{2}}{ }^{2}} \overrightarrow{\boldsymbol{u}} \overrightarrow{\boldsymbol{u}}\right)+\frac{1}{2} \nabla \cdot\left(\frac{n}{v_{T}{ }^{2}} \overrightarrow{\boldsymbol{u}} \overrightarrow{\boldsymbol{u}}\right) .
\end{aligned}
$$

Step 3. Comparing the first four right-hand terms in the equation above and Equation (82), we may suggest a modification of Equation (86) by introducing a coefficient of proportionality to the mass, $\frac{1}{v_{T}^{2}}$. This results in obtaining of the following equation:

$$
\begin{gathered}
\frac{\partial}{\partial t}\left[\frac{n}{v_{T}^{2}} \overrightarrow{\boldsymbol{u}}\right]+2 \frac{Z_{V}}{v_{T}^{2}} \overrightarrow{\boldsymbol{u}}+\frac{1}{2} \boldsymbol{\nabla}\left(\frac{n}{v_{T}^{2}} v_{T}^{2}\right)+\frac{1}{2} \boldsymbol{\nabla} \cdot\left[\frac{n}{v_{T}^{2}} \overrightarrow{\boldsymbol{u}} \overrightarrow{\boldsymbol{u}}\right]-2 \frac{n}{v_{T}^{2}} \overrightarrow{\boldsymbol{g}}+\frac{1}{m} \boldsymbol{\nabla}\left(\frac{p}{v_{T}^{2}}\right) \\
=P_{c} \boldsymbol{v}_{\text {rel }} \iiint_{\mathrm{V}} Z_{V} \frac{1}{v_{T}^{2}} Q_{i 0} \mathbf{G} d V^{\prime}+2 P_{c} \boldsymbol{v}_{\text {rel }} \iiint_{\mathrm{V}} Z_{V} \frac{1}{v_{T}^{2}} \frac{1}{v_{T}} Q_{i 0} \overrightarrow{\boldsymbol{u}} d V^{\prime} \\
+2 P_{c} \boldsymbol{v}_{\text {rel }} \iiint_{\mathrm{V}} Z_{V} \frac{1}{v_{T}^{2}} \frac{1}{v_{T}} Q_{i 0} \frac{1}{4 \pi} \frac{1}{\left|\overrightarrow{\boldsymbol{r}}-\overrightarrow{\boldsymbol{r}}^{\prime}\right|^{2}} \varphi_{i 0} \overrightarrow{\boldsymbol{g}} d V^{\prime}+P_{c} \boldsymbol{v}_{\text {rel }} \iiint_{\mathrm{V}} Z_{V} \frac{1}{v_{T^{2}}} \frac{1}{v_{T}^{2}} Q_{i 0}(\mathbf{G} \cdot \overrightarrow{\boldsymbol{u}}) \overrightarrow{\boldsymbol{u}} d V^{\prime} \\
+P_{c} \boldsymbol{v}_{\text {rel }} \iiint_{\mathrm{V}} Z_{V} \frac{1}{v_{T}^{2}} \frac{1}{v_{T}^{2}} Q_{i 0} \mathbf{G} \cdot\left[\varphi_{i 0}\left[(\overrightarrow{\boldsymbol{u}} \overrightarrow{\boldsymbol{g}}+\overrightarrow{\boldsymbol{g}} \overrightarrow{\boldsymbol{u}})+\overrightarrow{\boldsymbol{g}} \overrightarrow{\boldsymbol{g}} \varphi_{i 0}\right]\right] d V^{\prime},
\end{gathered}
$$




\section{(c) $(1) \Theta \Theta$}

Substituting the first five right-hand terms of Equation (96) by the left-hand terms of Equation (97) and rearranging terms, we finally obtain:

$$
\begin{aligned}
& \frac{\partial}{\partial t}\left[\frac{n}{v_{T}^{2}} \overrightarrow{\boldsymbol{u}}\right]+\boldsymbol{\nabla}\left(\frac{n}{v_{T}^{2}} v_{T}^{2}\right)+\boldsymbol{\nabla} \cdot\left[\frac{n}{v_{T}^{2}} \overrightarrow{\boldsymbol{u}} \overrightarrow{\boldsymbol{u}}\right]-\frac{n}{v_{T}^{2}} \overrightarrow{\boldsymbol{g}}+\frac{1}{m} \boldsymbol{\nabla}\left(\frac{p}{v_{T}^{2}}\right) \\
& =\nabla \cdot\left\{\frac{\mathbf{1}}{P_{c} \boldsymbol{v}_{\text {rel }}} \nabla\left\{\frac{\mathbf{1}}{P_{c} \boldsymbol{v}_{\text {rel }}}\left[\frac{\partial}{\partial t}[n \overrightarrow{\boldsymbol{u}}]+P_{c} \boldsymbol{v}_{\text {rel }} n \overrightarrow{\boldsymbol{u}}+\frac{1}{2} \boldsymbol{\nabla}\left(n v_{T}^{2}\right)+\frac{1}{2} \boldsymbol{\nabla} \cdot[n \overrightarrow{\boldsymbol{u}} \overrightarrow{\boldsymbol{u}}]-2 n \overrightarrow{\boldsymbol{g}}+\frac{1}{m} \boldsymbol{\nabla} p\right]\right\}\right\} \\
& -\frac{1}{2} \frac{\nabla\left(n v_{T}\right)}{n v_{T}} n-\frac{1}{2} \frac{\nabla\left(n v_{T}\right)}{n v_{T}} \cdot\left(\frac{n}{v_{T}^{2}} \overrightarrow{\boldsymbol{u}} \vec{u}\right) .
\end{aligned}
$$

Again, the equation above suggests that when considering the momentum balance, the thermal velocity of particles should be considered. This is reasonable for gases composing the plurality of randomly moving particles. Considering the conservation of mass by subtracting Equation (92) multiplied by $\overrightarrow{\boldsymbol{u}}$ from Equation (98) and rearranging terms, the following reduced vector-differential form of the momentum balance equation is provided:

$$
\begin{gathered}
\frac{\partial}{\partial t}[\overrightarrow{\boldsymbol{u}}]+\frac{v_{T}^{2}}{n} \nabla n+(\overrightarrow{\boldsymbol{u}} \cdot \boldsymbol{\nabla}) \overrightarrow{\boldsymbol{u}}+\frac{1}{m n} \boldsymbol{\nabla} p-\overrightarrow{\boldsymbol{g}} \\
=\frac{v_{T}^{2}}{n} \nabla \cdot\left\{\frac{\mathbf{1}}{P_{c} \boldsymbol{v}_{\boldsymbol{r e l}}} \nabla\left\{\frac{\mathbf{1}}{P_{c} \boldsymbol{v}_{\boldsymbol{r} \text { el }}}\left[\frac{\partial}{\partial t}[n \overrightarrow{\boldsymbol{u}}]+2 Z_{V} \overrightarrow{\boldsymbol{u}}+\frac{1}{2} \boldsymbol{\nabla}\left(n v_{T}^{2}\right)+\frac{1}{2} \boldsymbol{\nabla} \cdot[n \overrightarrow{\boldsymbol{u}} \overrightarrow{\boldsymbol{u}}]-2 n \overrightarrow{\boldsymbol{g}}+\frac{1}{m} \boldsymbol{\nabla} p\right]\right\}\right\} \\
-\frac{v_{T}^{2}}{n} \overrightarrow{\boldsymbol{u}} \boldsymbol{\nabla} \cdot\left\{\frac{\mathbf{1}}{P_{c} \boldsymbol{v}_{\boldsymbol{r} e l}} \nabla\left[\frac{\mathbf{1}}{P_{c} \boldsymbol{v}_{\boldsymbol{r} e l}}\left\{\frac{\partial}{\partial t} n+Z_{V}+\frac{1}{2} \boldsymbol{\nabla} \cdot[n \overrightarrow{\boldsymbol{u}}]\right\}\right]\right\}-\frac{1}{2} v_{T}^{2} \frac{\boldsymbol{\nabla}\left(n v_{T}\right)}{n v_{T}}+2 \frac{1}{m n} p \frac{\boldsymbol{\nabla} v_{T}}{v_{T}}
\end{gathered}
$$

Comparing the first, the second, the third, and the fourth left-hand terms of Equations (2) with the first, the third, the fourth, and the fifth left-hand terms of Equation (99), respectively, suggests that each pair of the corresponding compared terms is identical. The second left-hand term of the equation above is identified as a normalized self-diffusion force. Additional comparison of the right-hand terms of Equations (2) and (99) may suggest that stress inside the fluid has the following functional dependence:

$$
\begin{gathered}
\frac{1}{\rho} \boldsymbol{\nabla} \cdot \mathrm{T} \\
=\frac{v_{T}^{2}}{n} \nabla \cdot\left\{\frac{\mathbf{1}}{P_{c} \boldsymbol{v}_{r e l}} \nabla\left\{\frac{1}{P_{c} \boldsymbol{v}_{\boldsymbol{r} e l}}\left[\frac{\partial}{\partial t}[n \overrightarrow{\boldsymbol{u}}]+2 Z_{V} \overrightarrow{\boldsymbol{u}}+\frac{1}{2} \nabla\left(n v_{T}^{2}\right)+\frac{1}{2} \boldsymbol{\nabla} \cdot[n \overrightarrow{\boldsymbol{u}} \overrightarrow{\boldsymbol{u}}]-2 n \overrightarrow{\boldsymbol{g}}+\frac{1}{m} \nabla p\right]\right\}\right\} \\
-\frac{v_{T}^{2}}{n} \overrightarrow{\boldsymbol{u}} \boldsymbol{\nabla} \cdot\left\{\frac{\mathbf{1}}{P_{c} \boldsymbol{v}_{\text {rel }}} \nabla\left[\frac{\mathbf{1}}{P_{c} \boldsymbol{v}_{\text {rel }}}\left\{\frac{\partial}{\partial t} n+Z_{V}+\frac{1}{2} \boldsymbol{\nabla} \cdot[n \overrightarrow{\boldsymbol{u}}]\right\}\right]\right\}-\frac{1}{2} v_{T}^{2} \frac{\boldsymbol{\nabla}\left(n v_{T}\right)}{n v_{T}}+2 \frac{1}{m n} p \frac{\boldsymbol{\nabla} v_{T}}{v_{T}} .
\end{gathered}
$$

Since the vector differentiation of Equation (86) yields Equation (2) and Equation (86) defines $\overrightarrow{\boldsymbol{u}}$ at $t \geq 0$ as an implicit function of $\overrightarrow{\boldsymbol{r}}$ on $\mathbb{R}^{3}$, then Equation (86) is an implicit solution of Equation (2) in the region $\overrightarrow{\boldsymbol{r}} \in \mathbb{R}^{\mathbf{3}}$ occupied by the model gas.

For an incompressible model gas, Equation (99) is reduced to:

$$
\begin{gathered}
\frac{\partial}{\partial t}[\overrightarrow{\boldsymbol{u}}]+\overrightarrow{\boldsymbol{u}} \nabla \cdot[\overrightarrow{\boldsymbol{u}}]+\frac{1}{m n} \nabla p-\overrightarrow{\boldsymbol{g}} \\
=\frac{9}{16} \frac{\mathbf{1}}{P_{c}} \frac{\mathbf{1}}{P_{c}} v_{T}^{2} \nabla \cdot\left\{\frac{\mathbf{1}}{v_{T}} \nabla\left\{\frac{\mathbf{1}}{v_{T}}\left[\frac{\partial}{\partial t}[\overrightarrow{\boldsymbol{u}}]+P_{c} \boldsymbol{v}_{r e l} \overrightarrow{\boldsymbol{u}}+\frac{1}{2} \nabla\left(v_{T}{ }^{2}\right)+\frac{1}{2} \nabla \cdot[\overrightarrow{\boldsymbol{u}} \overrightarrow{\boldsymbol{u}}]-2 \overrightarrow{\boldsymbol{g}}\right]\right\}\right\} \\
-\frac{1}{2} v_{T}^{2} \frac{\boldsymbol{\nabla}\left(v_{T}\right)}{v_{T}}-\frac{9}{16} \frac{\mathbf{1}}{P_{c}} \frac{\mathbf{1}}{P_{c}} v_{T}^{2} \overrightarrow{\boldsymbol{u}} \boldsymbol{\nabla} \cdot\left\{\frac{\mathbf{1}}{v_{T}} \nabla\left[\frac{\mathbf{1}}{v_{T}} \frac{1}{2} \boldsymbol{\nabla} \cdot[\overrightarrow{\boldsymbol{u}}]\right]\right\},
\end{gathered}
$$

which is obtained by considering that $P_{c}$ and $n$ are constants. 
Technically, with the initial state $\overrightarrow{\boldsymbol{u}}_{\mathbf{0}}(\overrightarrow{\boldsymbol{r}})$ at time $t=0$, one upon resolving Equation (101) may determine the evolution or dynamics of relaxation of the fluid flow motion for $t>0$. However, even with this significant reduction of the Navier-Stokes equation (incompressibility), the direct mathematical solution of the equation above is very difficult or rather impossible.

However, the alternative integral balance equations such as Equation (82) and Equation (86), which are implicit solutions for vector-differential Equations (92) and Equation (101), respectively, can be solved by computer-implemented methods with no difficulties and will need the shorter computational time. Detailed analysis of direct resolving of one-dimensional integral balance equations by a method of sequential approximations can be found in our recent nonprovisional patent application [11] and PCT application [12].

\section{Validation Tests}

The following five validation tests A1, A2, A3, A3.2, and A4, are aimed to demonstrate the feasibility of the proposed method.

A1 Determining the total rate of collisions per unit area on a surface being in contact with the gas

Fig. 6 is a perspective view for explaining a method for determining the total rate of collisions per unit area on a surface being in contact with the gas. In a system with no gravitational force, the ballistic particles have straight-line trajectories. Here we limit further our consideration to an incompressible model gas at the uniform temperature in a stable condition. In the semi-sphere filled with the model gas over the being at rest surface $A_{s}$ having a directional vector $\overrightarrow{\boldsymbol{n}}$, each particle having an instantaneous randomly directed vector of thermal of magnitude, $v_{\mathrm{T}}$, may have the instant vector-velocity component directing a particle toward the surface $A_{s}$. In Fig. 6 , the particle 601 positioned at $\overrightarrow{\boldsymbol{r}}^{\prime}$ is shown at distance $y$ from the surface $A_{s}$ in point $\overrightarrow{\boldsymbol{r}}$. The angle between the instant vector-velocity $\overrightarrow{\boldsymbol{v}}$ and the directional vector $\overrightarrow{\boldsymbol{n}}$ is labeled as $\theta$. The ends of the directionally random vector-velocity of magnitude $v_{\mathrm{T}}$ form spherical surface 602 .

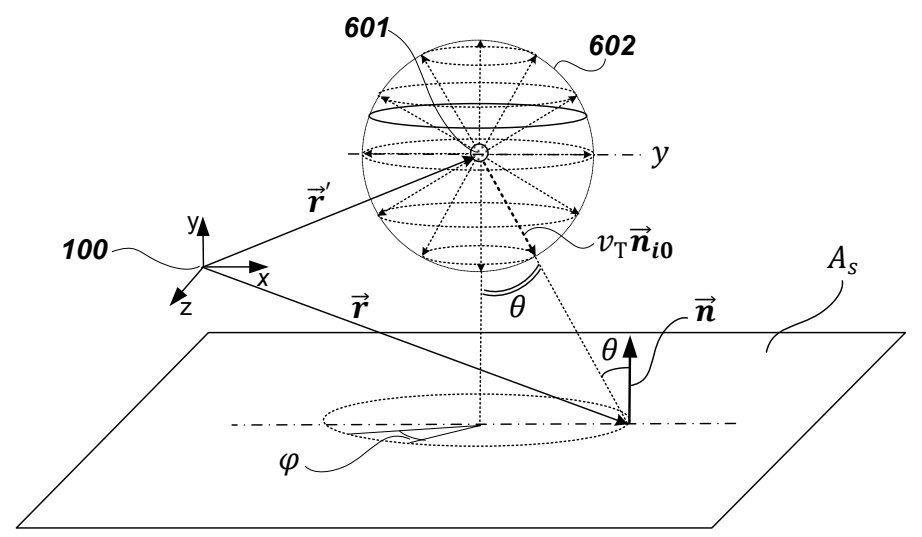

Fig. 6 The perspective view of the geometry and coordinate system for determining the total rate of collisions per unit area and the pressure exerted on the surface from the gas volume

Adopting Equation (37) to the conditions above and assigning $\boldsymbol{\Psi}_{\boldsymbol{i n}}=1$, the total rate of collisions per unit area on the surface $A_{s}$ is given as: 


$$
Z=-Z_{V} \frac{1}{4 \pi} \iiint_{V} \frac{1}{\left|\overrightarrow{\boldsymbol{r}}-\overrightarrow{\boldsymbol{r}}^{\prime}\right|^{2}} Q_{i}\left(\overrightarrow{\boldsymbol{r}}, \overrightarrow{\boldsymbol{r}}^{\prime}\right) \overrightarrow{\boldsymbol{n}}_{\boldsymbol{i 0}} \cdot \overrightarrow{\boldsymbol{n}} d V^{\prime}
$$

where

$$
\begin{gathered}
\overrightarrow{\boldsymbol{n}}_{\boldsymbol{i} \mathbf{0}}=\frac{\overrightarrow{\boldsymbol{r}}-\overrightarrow{\boldsymbol{r}}^{\prime}}{\left|\overrightarrow{\boldsymbol{r}}-\overrightarrow{\boldsymbol{r}}^{\prime}\right|}, \\
Q_{i}\left(\overrightarrow{\boldsymbol{r}}, \overrightarrow{\boldsymbol{r}}^{\prime}\right)=\exp \left(-\frac{4}{3} P_{c}\left|\overrightarrow{\boldsymbol{r}}-\overrightarrow{\boldsymbol{r}}^{\prime}\right|\right),
\end{gathered}
$$

and $Z_{V}$ is the rate of collisions per unit volume, which, upon substitution of Equation (28) in Equation (80) is expressed as

$$
Z_{V}=\frac{2}{3} n P_{c} v_{T}
$$

where $n$ is particle density, $v_{T}$ is the magnitude of the thermal velocity, and $P_{C}$ is the average number of collisions per unit length.

Finally, using the spherical polar coordinate system, substituting Equations (103), (104), and (105) in Equation (102), and considering that

$$
\left|\overrightarrow{\boldsymbol{r}}-\overrightarrow{\boldsymbol{r}}^{\prime}\right|=y / \cos \theta
$$

and

$$
\overrightarrow{\boldsymbol{n}}_{\boldsymbol{i 0}} \cdot \overrightarrow{\boldsymbol{n}}=-\cos \theta,
$$

we convert Equation (102) and calculate as:

$$
Z=\frac{2}{3} n P_{c} v_{T} \frac{1}{4 \pi} \int_{0}^{\frac{\pi}{2}} \int_{0}^{2 \pi} \int_{0}^{\infty} \exp \left(-\frac{4}{3} \frac{P_{c} y}{\cos \theta}\right) \sin \theta d \theta d \varphi d y=n \frac{v_{T}}{4},
$$

That the result of derivation above according to our method, which is applied to the model gas, is identical to the result of the derivation of the rate of collisions per unit area of an ideal gas, which one can find in any course of the kinetic theory of gases, supports the Ballistic Model.

A2 Determining the pressure exerted on the surface from the entire gas volume being in contact with the surface

Referring to the previous section and Fig. 6, we recognize that each particle of the model gas carries the momentum

$$
\overrightarrow{\boldsymbol{p}}=m v_{\mathrm{T}} \overrightarrow{\boldsymbol{n}}_{\boldsymbol{i 0}}
$$

and the momentum component delivered by the particle to the surface $A_{s}$ is

$$
\Delta \boldsymbol{p}_{\boldsymbol{y}}=m v_{\mathrm{T}} \overrightarrow{\boldsymbol{n}}_{\boldsymbol{i 0}} \cdot \overrightarrow{\boldsymbol{n}},
$$

where $m$ is mass of the particle/molecule.

If the particle undergoes an elastic collision with the surface, in such a collision, the momentum passed on the surface is doubled. Adopting Equation (37) to the conditions listed initially referring to Figure 7 and assigning $\Psi_{\boldsymbol{i n}}=2 \Delta \boldsymbol{p}_{\boldsymbol{y}}$, the pressure $P$ exerted on the surface $A_{s}$ is given as: 


$$
P=Z_{V} \frac{2}{4 \pi} m v_{\mathrm{T}} \iiint_{\mathrm{V}} \frac{1}{\left|\overrightarrow{\boldsymbol{r}}-\overrightarrow{\boldsymbol{r}}^{\prime}\right|^{2}} Q_{i}\left(\overrightarrow{\boldsymbol{r}}, \overrightarrow{\boldsymbol{r}}^{\prime}\right)\left(\overrightarrow{\boldsymbol{n}}_{\boldsymbol{i 0}} \cdot \overrightarrow{\boldsymbol{n}}\right)\left(\overrightarrow{\boldsymbol{n}}_{\boldsymbol{i 0}} \cdot \overrightarrow{\boldsymbol{n}}\right) d V^{\prime} .
$$

Now, using the spherical polar coordinate system, substituting Equations (103), (104), (105), and (106) in Equation (111), we convert Equation (111) and calculate as:

$$
P=\frac{2}{3} n P_{c} v_{T} \frac{1}{\pi} m v_{\mathrm{T}} \int_{0}^{\pi / 2} \int_{0}^{2 \pi} \int_{0}^{\infty} \exp \left(-\frac{4}{3} \frac{P_{c} y}{\cos \theta}\right) \cos ^{2} \theta \sin \theta d \theta d \varphi d y=\frac{m n v_{T}^{2}}{3} .
$$

Again, the result of derivation above according to our method, which considers an impact on the surface of unlimited number particles of the model gas (integration along $y$ direction from zero to infinity), is identical to the result of derivation of the pressure of a perfect gas according to the kinetic theory of gases, which corroborates with the Ballistic Model.

In addition, analyzing examples A1 and A2 above, one may note that the proposed method provides the possibility of quantifying impacts of the limited number of particles on the gassolid interface. This, from the practical viewpoint, is important in many applications dealing with MEMS technology (capacitive sensing, electrostatic actuation mechanisms, a block of sensing mass in micro gyroscopes, accelerometers, switches, mirrors, pressure sensors and so on) [16].

A short communication about these two examples (A3 and A4) is present on the website and can be accessed on [17]. This communication is not published nor is under publication elsewhere.

A3 Incompressible model gas flow between two infinite parallel plates at the uniform temperature in a case of diffuse particle scatterings

The velocity profile induced in the model gas due to the pressure gradient along the channel is analyzed by an analytical method based on the proposed model gas flow described above. Fig. 7 shows schematically a one-dimensional model gas system, in which the main control volume $(\mathrm{CV})$ of the unit length volume $d \mathrm{~V}=\Delta x \Delta y$ is at $\boldsymbol{y}$ within the model gas flow confined by two parallel plates at $\boldsymbol{y}=0$ and $\boldsymbol{y}=H$. Plate 1 and Plate 2 confine across $y$-axis a model gas flow along the $\mathrm{x}$-axis and surfaces 701 and 702 positioned at distance $\Delta x$ bound a portion of the model gas system along the $\mathrm{x}$-axis. The known method of obtaining analytical solutions for isothermal gaseous flow with slip boundary conditions is based on the locally fully developed flow assumption and applying the second-order velocity slip boundary conditions (Maxwelltype assumption) in the following form [18]

$$
u-u_{w}= \pm C_{1} \lambda_{f} \frac{\partial u}{\partial y}-C_{2} \lambda_{f}^{2} \frac{\partial^{2} u}{\partial y^{2}},
$$

where $u$ is the gas slip velocity near the wall, $u_{w}$ is the tangential velocity of the wall, and $\lambda_{f}$ is the mean free path.

The method described below uses none of the Maxwell-type assumptions.

\section{A3.1 Properties and features of the gas-solid interface}

Here we assign the following additional special properties to the model gas being in contact with a gas-solid interface revealing purely diffuse scattering of particles: 
(1) Each of a plurality of collisions on a gas-solid interface of the model gas system, which has resulted in diffuse particle scattering from the gas-solid interface, is treated as an act of interaction involving a property transfer from the gas-solid interface to a scattered particle.

(2) Each of a plurality of points of diffuse particle scattering on the gas-solid interface is treated as a heterogeneous point source for each of a plurality of scattered particles.

(3) The velocity of each of a plurality of heterogeneous point sources on the gas-solid interface is assigned to be equal to the velocity of the gas-solid interface in each of a plurality of corresponding points of diffuse particle scattering.

(4) The point source strength of each of the plurality of heterogeneous point sources on the gas-solid interface is assigned to be directly proportional to a property accommodation coefficient $\sigma$ intrincsic to a corresponding point of the gas-solid interface at the time of diffuse particle scattering.

Note: Diffuse scattering is an act of interaction involving property transfer from a gas-solid interface to a scattered particle. Specular scattering does not involve property exchange between the gas-solid interface and a scattered particle.

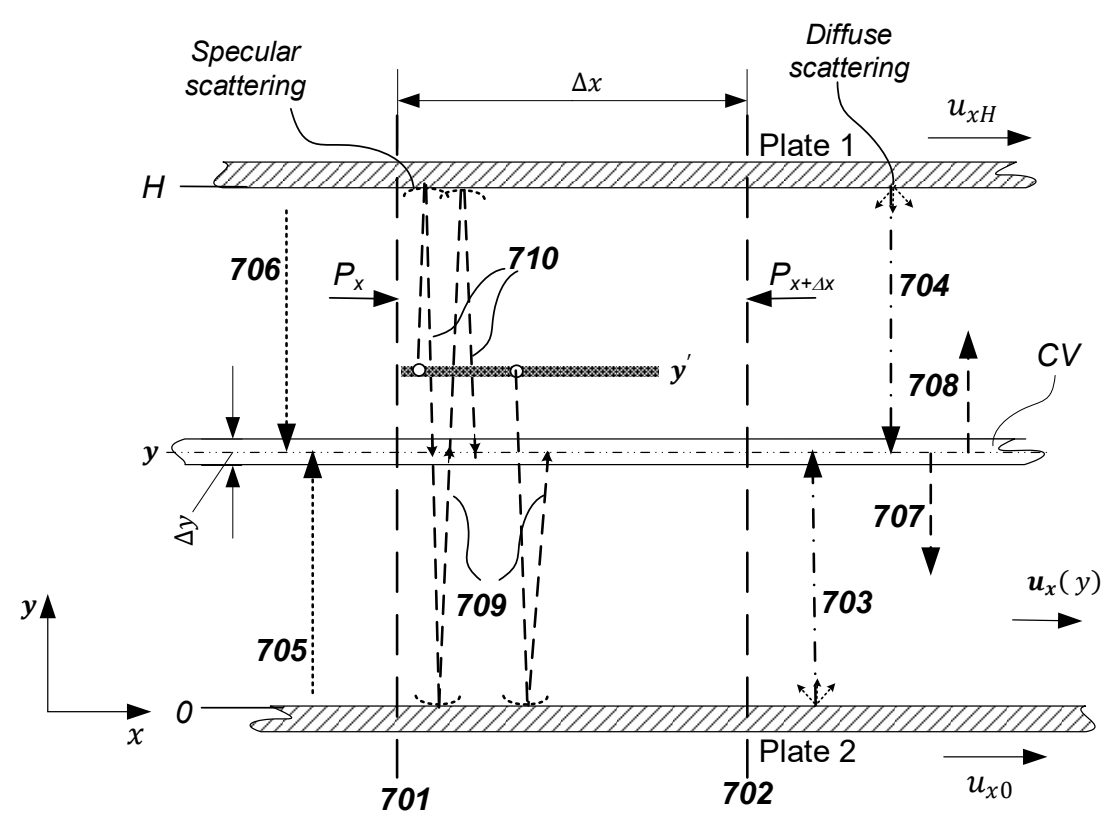

Fig. 7 The schematic one-dimensional view of flow geometry and coordinate system showing model gas flow confined between two parallel plates

In the model gas system having gas-solid interfaces with the purely diffuse scattering of particles, each particle originated from preceding diffuse scatterings from the interfaces delivers in the CV some property obtained from the location of the original diffuse scattering (ballistic trajectory 703 from plate 2 and ballistic trajectory 704 from plate 1 of Fig. 7). Ballistic trajectories 705 and 706 show movement of ballistic particles, which are originated from the preceding collisions in the space occupied by the model gas, into targeting point $\boldsymbol{y}$. Finally, ballistic trajectories 707 and 708 show the movement of diverging ballistic particles, which are originated from the preceding collisions in space surrounding point $\boldsymbol{y}$. The fluid flow, with the 
velocity distribution $\boldsymbol{u}_{\boldsymbol{x}}(y)$, is forced by both the pressure gradient $\frac{d P}{d x}$ and the plates' movement with velocity $\boldsymbol{u}_{\boldsymbol{x} \mathbf{0}}$ and $\boldsymbol{u}_{\boldsymbol{x} \boldsymbol{H}}$ along the x-direction.

\section{A3.2 Direct validation of the BPPBS in the gas space confined between two parallel plates}

Recognizing that in a steady-state model incompressible gas flow at the uniform temperature, all variables describing flow depend on the position in space and are not dependent on time, so that $n$, the particle density, $m$ and $v_{T}$, the mass and the magnitude of the thermal velocity, respectively, of the particle/molecule are constant. In the model gas system confined by the infinite parallel plates being at rest along $y$ direction and characterized by the above conditions, the mass-flow velocity along $y$ direction is expected to be zero. Now, considering that point $y$ is not included in integration, we may formulate the system of mass-balance and momentum-balance integral equations, respectively, in these forms:

$$
\begin{gathered}
Z_{V} m=\frac{1}{2} P_{c} Z_{V} m \int_{\mathbf{0}}^{\mathbf{H}} \exp \left(-P_{c}\left|\boldsymbol{y}-\boldsymbol{y}^{\prime}\right|\right) d y^{\prime} \\
+Z_{b} P_{c} m \exp \left(-P_{c}(H-y)\right)+Z_{b} P_{c} m \exp \left(-P_{c} y\right),
\end{gathered}
$$

for mass-balance, and

$$
\begin{gathered}
0=\frac{1}{2} P_{c} Z_{V} v_{T} m \int_{\mathbf{0}}^{\mathbf{H}} \exp \left(-P_{c}\left|\boldsymbol{y}-\boldsymbol{y}^{\prime}\right|\right) \boldsymbol{n}_{+\mathbf{0}} d y^{\prime} \\
-Z_{b} P_{c} v_{T} m \exp \left(-P_{c}(H-y)\right)+Z_{b} P_{c} v_{T} m \exp \left(-P_{c} y\right),
\end{gathered}
$$

for $y$-momentum-balance. Here $P_{c}$ is the average number of collisions per unit length, $Z_{b}$ is the rate of collisions per unit area on plate 1 or plate 2 , and $Z_{V}$ is the rate of collisions per unit volume, which is defined as

$$
Z_{V}=\frac{1}{2} n P_{c} v_{T}
$$

and $\boldsymbol{n}_{+\mathbf{0}}$ is the unit vector of arbitrary direction from the point $y$, which is defined as

$$
n_{+0}=\frac{y-y^{\prime}}{\left|y-y^{\prime}\right|} \text {. }
$$

The left-hand of Equation (115) has zero value because of the average momentum of the diverging particle, which is measured by the instant momentum $m v_{T} \boldsymbol{n}_{+\mathbf{0}}$, is zero.

Solving Equation (114) will cause finding that

$$
Z_{b}=\frac{Z_{V}}{2 P_{c}}
$$

Substituting Equation (118) in Equation (115), we may verify that the mass and momentum balance in any point of space between plate 1 and plate 2 along $y$ axis are concerved, which suggests the valididty of the BPPBS in the one-dimensional configuration.

A3.3 Analytical derivation of the velocity profile induced in the model gas due to the pressure gradient along the channel between two parallel plates

Step 1. Formulating an integral form of the $\boldsymbol{u}_{\boldsymbol{x}}$-momentum balance equation 


\section{(c) $(1)(\Theta \Theta$}

Considering the above, we obtain the following integral form of the $\boldsymbol{u}_{\boldsymbol{x}}$ momentum balance equation in a stable model gas flow:

$$
\begin{gathered}
Z_{V} \boldsymbol{u}_{\boldsymbol{x}}(y) m=-\frac{d P}{d x}+\frac{1}{2} Z_{V} P_{c} m \int_{\mathbf{0}}^{\mathbf{H}} \exp \left(-P_{c}\left|\boldsymbol{y}-\boldsymbol{y}^{\prime}\right|\right) \boldsymbol{u}_{\boldsymbol{x}}\left(y^{\prime}\right) d y^{\prime} \\
+\frac{1}{2} Z_{V} \boldsymbol{u}_{\boldsymbol{x} \boldsymbol{H}} m \exp \left(-P_{c}(H-y)\right)+\frac{1}{2} Z_{V} \boldsymbol{u}_{\boldsymbol{x} \mathbf{0}} m \exp \left(-P_{c} y\right),
\end{gathered}
$$

Step 2. Obtaining a differential form matching to the corresponding integral form of the $\boldsymbol{u}_{\boldsymbol{x}}$ momentum balance equation

Here we use the method of differentiation (twice). Each step of the differentiation is followed by the step of subsequent elimination of the integral terms by using the original equation, which is given as:

$$
m \frac{Z_{V}}{P_{c}^{2}} \frac{d^{2}}{d y^{2}} \boldsymbol{u}_{\boldsymbol{x}}=\frac{d P}{d x}
$$

Step 3. Obtaining a general solution having arbitrary coefficients

Integrating twice the equation above, we obtain:

$$
\boldsymbol{u}_{\boldsymbol{x}}(y)=A y^{2}+B y+C,
$$

where

$$
A=\frac{P_{c}^{2}}{2 m} \frac{1}{Z_{V}} \frac{d P}{d x}=\frac{P_{c}}{m n v_{T}} \frac{d P}{d x}
$$

and $B$ and $C$ are the arbitrary coefficients.

Step 4. Determining the values of each of the arbitrary coefficients

Introducing Equation (121) in Equation (119) (for a specific number of points (two) within the model gas system), we determine the values of each of the arbitrary coefficients. For certainty, we selected points $y=0$ and $y=H$ for the balance establishment.

Step 5. Solving a system of the obtained in Step 4 algebraic equations

We obtained the following functional relationship of fluid velocity $\boldsymbol{u}_{\boldsymbol{x}}$ from other properties and geometry parameters characterizing the model gas system:

$$
B=-A H+\frac{P_{c}\left(u_{x H}-u_{x 0}\right)}{P_{c} H+2}
$$

and

$$
C=-A\left(\frac{2}{P_{c}{ }^{2}}+\frac{H}{P_{c}}\right)+\frac{u_{x H}}{P_{c} H+2}+\frac{u_{x 0}}{P_{c} H+2}\left[P_{c} H+1\right] .
$$

Where Plate 1 and Plate 2 are at rest, i.e. $u_{x H}=u_{x 0}=0$, substitution in Equation (121) of Equations (122), (123), and (124), in which $P_{c} H$ is replaced by $K n^{-1}$, will yield:

$$
\boldsymbol{u}_{x}(y)=-\frac{H}{m n v_{T}} \frac{d P}{d x} \frac{1}{K n}\left(-\frac{y^{2}}{H^{2}}+\frac{y}{H}+K n+2 K n^{2}\right),
$$

where $K n$ is the Knudsen number defined as the ratio of the mean free path $\lambda_{f} \sim 1 / P_{c}$ and the representative length scale $H$, i.e., $K n=\lambda_{f} / H$. Further integration of $\boldsymbol{u}_{\boldsymbol{x}}(y)$ in $y$-direction followed by normalization with $-\frac{H}{m n v_{T}} \frac{d P}{d x}$ results in an expression for a non-dimensional flow rate $Q_{N}$ : 


$$
Q_{N}=\frac{1}{K n}\left(\frac{1}{6}+K n+2 K n^{2}\right),
$$

The equation above reveals there is a minimum in the normalized mass flow rate (at about $K n \cong 0.3$ ), which is called the Knudsen paradox in the literature [19]. It is explained by the fact that " $[\mathrm{w}]$ ith increasing Knudsen number, the additional diffusive flux becomes significant and deviates from the no-slip solution. Beyond a critical limit $(K n>0.3)$, the diffusive flux dominates the convective flux [19].

Fig. 8 compares the non-dimensional flow rates calculated by Equation (126) for the proposed model (Ballistic model) and a selection of slip models proposed by various authors. The nondimensional flow rate was proposed in the form [20]

$$
Q_{N}=\sqrt{\pi}\left(1+6 C_{1} K n+12 C_{2} K n^{2}\right) /(12 K n),
$$

where corresponding slip coefficients $C_{1}$ and $C_{2}$ are taken from Table 6 [21]. The results of the linearized Boltzmann solution of [22] are plotted from Figure 2 of [23]. In Fig. 8, the flow rate according to Equations (126) is rescaled by $\sqrt{\pi} / 2$ factor for the comparison purposes.

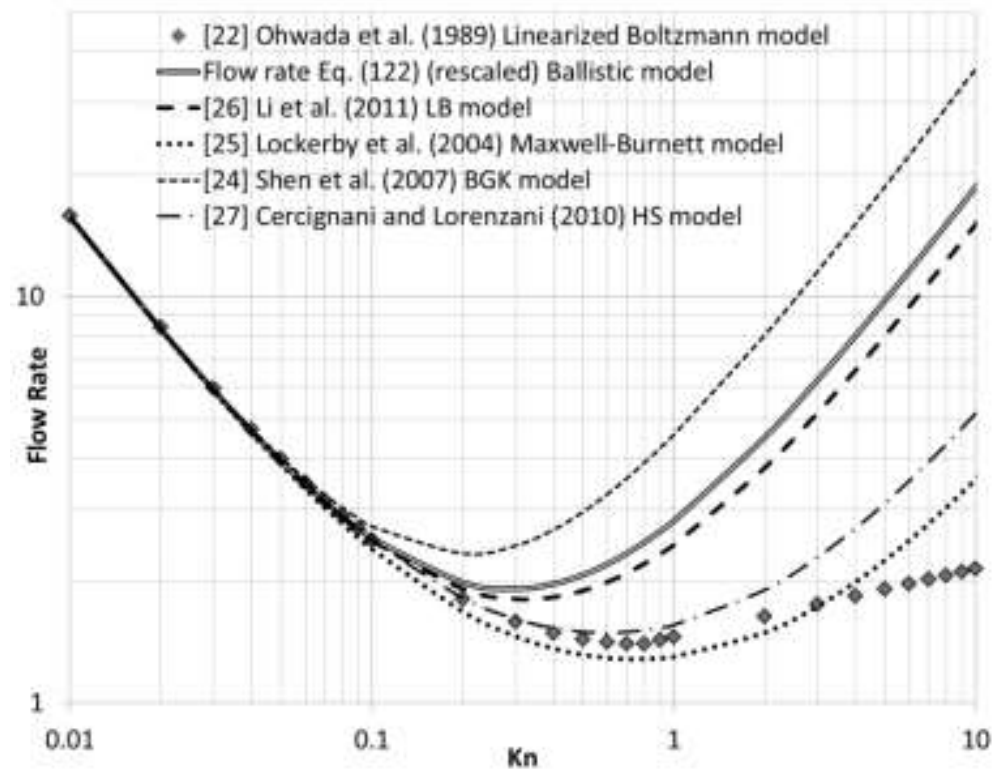

Fig. 8. Comparison of the non-dimensional flow rate $Q_{H}$ as a function of the Knudsen number for the proposed model and a selection of slip models

While comparing the non-dimensional flow rates calculated by Equation (126) for the Ballistic Model and a selection of slip models proposed by various authors [22], [24], [25], [26], and [27], we note that the discrepancy among various slip models, including the model presented here, is small for $K n<0.1$. However, the significant discrepancy occurs for $K n>$ 0.1. Still, the results obtained according to our Ballistic Model are within the reasonable range of discrepancies of variety of the literature data (see also Table 6 in [21]).

A4 Incompressible model gas flow in the space between two being at rest infinite parallel plates in the open channel at the uniform temperature in a case of mixed diffuse and specular particle scatterings 
Here we assume the symmetricity of the expected solution (because of the zero velocity of the confining plates). We also consider that the influx of $\boldsymbol{u}_{\boldsymbol{x}}$-momentum in a given point $y$ is formed by converging ballistic particles originated from initial collisions within the model gas volume and having at least the last preceding specular scattering either from the gas-solid interface of Plate 2 (709) or from the gas-solid interface of Plate 1 (710). With these assumptions, we may obtain the following integral form of the $\boldsymbol{u}_{\boldsymbol{x}}$ momentum balance equation in a stable model gas flow:

$$
\begin{gathered}
m Z_{V} \boldsymbol{u}_{\boldsymbol{x}}=-\frac{d P}{d x}+\frac{1}{2} P_{c} m Z_{V} \int_{\mathbf{0}}^{\mathrm{H}} \exp \left(-P_{c}\left|\boldsymbol{y}-\boldsymbol{y}^{\prime}\right|\right) \boldsymbol{u}_{\boldsymbol{x}}\left(y^{\prime}\right) d y^{\prime} \\
+\frac{1}{2} P_{c} \frac{(1-\sigma) \exp \left(-P_{c} \mathrm{H}\right)\left[\exp \left(-P_{c}(H-y)\right)+\exp \left(-P_{c} y\right)\right]}{1-(1-\sigma) \exp \left(-P_{c} H\right)} m Z_{V} \int_{\mathbf{0}}^{\boldsymbol{H}} \boldsymbol{u}_{\boldsymbol{x}}\left(\boldsymbol{y}^{\prime}\right) \exp \left(P_{c} y^{\prime}\right) \mathrm{dy}^{\prime}
\end{gathered}
$$

where $\sigma$ is the momentum accommodation coefficient, which is the probability, for an incident particle, to accommodate momentum from the gas-solid interface and to scatter back in the model gas as a diffuse particle. Comparative analysis of the integral equations describing model gas flow confined between the parallel plates with purely diffuse scattering (see Equation (119)) and mixed diffuse and specular scattering (see Equation (128)) results in finding they have similar forms if

$$
u_{x H}=u_{x 0}=P_{c} \exp \left(-P_{c} \mathrm{H}\right) \frac{(1-\sigma)}{1-(1-\sigma) \exp \left(-P_{c} H\right)} \int_{\mathbf{0}}^{\boldsymbol{H}} u_{x}\left(\boldsymbol{y}^{\prime}\right) \exp \left(P_{c} \boldsymbol{y}^{\prime}\right) \mathrm{dy}^{\prime},
$$

where $u_{x H}$ and $u_{x 0}$ represent coefficients of Equation (119), and the right-hand of the equation above represents the analogous coefficient in Equation (128). The analysis also leads to the conclusion that the velocity profile can be described by Equation (121), where coefficient $A$ is expressed by Equation (122). Substituting Equation (129) in Equation (123), we obtain:

$$
B=-A H \text {. }
$$

Finally, substituting $u_{x}\left(\boldsymbol{y}^{\prime}\right)=A\left(y^{\prime}\right)^{2}+B y^{\prime}+C$ in Equation (129) and substituting the resulting equation in Equation (124), then executing corresponding integrations and algebra operations, we obtain:

$$
C=-A H^{2}\left[\frac{2-\sigma}{\sigma} K n+2 K n^{2}\right] .
$$

Remarkably, the derived tangential slip velocity coefficient $C$ contains the term being proportional to $\frac{2-\sigma}{\sigma}$, which was not indirectly introduced by applying the Maxwell-type assumptions to the slip-velocity boundary conditions.

Substitution in Equation (121) of Equations (122), (130), and (131) will yield:

$$
\boldsymbol{u}_{\boldsymbol{x}}(y)=-\frac{H}{m n v_{T}} \frac{d P}{d x} \frac{1}{K n}\left(-\frac{y^{2}}{H^{2}}+\frac{y}{H}+\frac{2-\sigma}{\sigma} K n+2 K n^{2}\right) .
$$

That the tangential slip velocity coefficient $C$ is analytically defined from the continuum through the slip and transition to free-molecule flow regimes gave us confidence that our approach is valid. Again, our method uses none of the Maxwell-type assumptions. Still, coefficient $C$ contains the terms proportional to $K n$ and $K n^{2}$ in Equation (125) or Equation (132). 
In addition, we need to note we provided the above to demonstrate the feasibility of our Ballistic Model. These results may be improved by considering the compressibility of the gas. However, obtaining the analytical representation of the velocity profile with this complication may be a challenge, if not impossible.

\section{Conclusions}

(1) A physical approach to finding implicit solutions for the Navier-Stokes equations is presented here. Instead of modeling based on using an infinitesimal fluid element, which is treated as a continuous medium, fluid flow is approximated as a model gas flow in a model gas system being identical to the fluid system. The physical approach is based on if each of a plurality of particles, which compose the model gas, travels with a probability between any of two points in space occupied by the model gas and follows a ballistic trajectory governed by a law of motion in free space and on treating each of the plurality of ballistic particles as a property carrier transporting one or more of mass, momentum, and energy between the points of consecutive collisions.

(2) According to the physical approach, each point in space occupied by the model gas is treated as both a sink accumulating property delivered by converging ballistic particles from the entire model gas system and a source dispersing property by diverging ballistic particles, a general integrodifferential form of the property balance equation is proposed. The general integro-differential form of the property balance is further adopted to derive the integro-differential forms of mass balance, momentum balance, and energy balance.

(3) The physical approach is validated by demonstrating that, in the collision-dominated flow regime, the differential equations, which we converted from the derived integro-differential mass and momentum balance equations, are identical to the corresponding Navier-Stokes equations. This finding supports the conclusion that, in the collision-dominated flow regime, the formulated integrodifferential forms of the balance are exact implicit solutions for corresponding Navier-Stokes equations. It also suggests that a physical problem described by Navier-Stokes equations may be represented by the proposed matching vector integro-differential forms of the mass, momentum, and energy balance, which can be solved by computer-implemented methods with no difficulty. Finally, the formulated integro-differential forms of the mass, momentum, and energy balances are advantageous over the Navier-Stokes equations since they are operable from the continuum flow regime to the rarefied flow regime.

(4) From the fundamental viewpoint, we have discovered a new principle of the property (mass, momentum, energy, and so on) balance in space, which we have named the Ballistic Principle of the Property Balance in Space. The direct test supporting the validity of the BPPBS is performed by demonstration of the mass-balance and momentum-balance conservation in a given non-moving point in one-dimensional incompressible gas space confined between two parallel plates at the uniform temperature by formulating and computing the balance between the rate of mass and momentum influx and the rate of mass and momentum efflux, respectively, in the given point of the gas space.

(5) We demonstrated the analytical solution for determining the velocity profile induced in the model gas flow due to the pressure gradient along the channel. The derivation is based on the proposed model gas properties and the Ballistic Model. That the analytical solution is valid to describe 
the velocity profile in the wide range of gas pressure from the continuum through the slip and transition to free-molecule flow regimes gave us additional confidence that our approach is valid.

(6) From the practical viewpoint, the proposed method can be a fundamental base of a new generation of the CFD software that does not use the Navier-Stokes solver; thus, eliminating uncertainty, improving predictability, and shortening the computational time.

\section{REFERENCES}

[1] "https://www.grc.nasa.gov/www/k-12/airplane/nseqs.html," 05 May 2015. [Online].

[2] P. Lemarie-Rieusset, The Navier-Stokes Problem in the 21 st Century (Chapman and Hall/CRC, New York, 2016)., New York: Chapman and Hall/CRC, 2016.

[3] C. Fefferman, Existence and smoothness of the Navier-Stokes equation, J. Carlson, Ed., Providence: American Mathematical Society, 2006.

[4] T. Tao, "https://terrytao.wordpress.com/2007/03/18/why-global-regularity-for-navier-stokes-ishard/," 2007. [Online].

[5] G. Karnidakis and A. Beskok, Microflows: Fundamentals and Simulation, New York: Springer, 2001.

[6] M. Gad-el-Hak, "Flow Physics," in Handbook of MEMS, M. Gad-el-Hak, Ed., Boca Raton, FL, CRC, 2002.

[7] N. Hadjiconstantinoua, "The limits of Navier-Stokes theory and kinetic extensions for describing small-scale gaseous hydrodynamics," Phys. Fluids 18, p. 111301, 2006.

[8] R. Benzi, S. Sicci and M. Vergassola, "The lattice Boltzmann equation: theory and applications," Physics Reports, vol. 222, no. 3, pp. 145-197, 1992.

[9] F. Tosi and S. Succi, "AN INTRODUCTION TO ENTROPIC LATTICE," SIMAI e-Lecture Notes ISSN 1970-4429, vol. 1, pp. 2-42, 2008.

[10] H. Chen, J. Hoch Jr. and C. Teixeira, "Computer simulation of physical processes". US Patent 6089744, 18072000.

[11] N. Kislov, "Analytical Tools and Methods for Modeling Transport Processes in Fluids." US Patent 10467362 B2, 5 November 2018.

[12] N. Kislov, "Analytical Tools and Method for Modeling Transport Processes in Fluids." International Patent Publication: WO 2019/143428 A1, 14 December 2018.

[13] J. Plawsky, Transport Phenomena Fundamentals, 2nd ed., Boca Raton: CRC, 2010, p. 8.

[14] M. Tenenbaum and H. Pollard, Ordinary differential equations, 2nd ed., New York: Dover Publications, Inc., 1985.

[15] A. D. Polyanin and A. Manzhirov, Handbook of Integral Equations, 2nd ed., Chapman and Hall: CRC, 2008, p. 564.

[16] W. Wang, J. Jia, and J. Li, "Slide film damping in microelectromechanical system devices," $J$ Nanoengineering and Nanosystems, vol. 227, no. 4, pp. 162-170, 2013. 
[17] N. Kislov, "https://contest.techbriefs.com/2019/entries/aerospace-and-defense/9789-0627-134220analytical-tools-and-method-for-next-generation-computational-fluid-dynamics.," Tech Brief, 2706 2019. [Online].

[18] G. Tang, Y. He and W. Tao, "Comparison of gas slip models with solutions of linearized Boltzmann equation and direct simulation of Monte Carlo method," International Journal of Modern Physics C, vol. 18, no. 2, pp. 203-216, 2007.

[19] N. Dongari, Dadzie, Z. Y. S.K. and J. Reese, "Isothermal micro-channel gas flow using a hydrodynamic model with dissipative mass flux," AIP Conf. Proc., vol. 1333, pp. 718-723, 2011.

[20] N. Hadjiconstantinou and O. Simek, "Constant-wall-temperature Nusselt number in micro and nanochannels," ASME J Heat, vol. 124, pp. 356-364, 2002.

[21] W. Zhang, G. Meng and X. Wei, "A review on slip models for gas microflows," Microfluidics and Nanofluidics, vol. 13, no. 6, p. 845-882, 2012.

[22] T. Ohwada, Y. Sone and K. Aoki, "Numerical analysis of the Poiseuille and thermal transpiration flows between parallel plates on the basis of the Boltzmann equation for hard-sphere molecules.," Phys. Fluids A, vol. 1, p. 2042, 1989.

[23] G. Tang, Y. He and W. Tao, "Comparison of gas slip models with solutions of linearized Boltzmann equation and direct simulation of Monte Carlo method," International Journal of Modern Physics C, vol. 18, no. 2, p. $203\{216,2007$.

[24] S. Shen, G. Chen, R. Crone and M. Dufresne, "A kinetic-theory based first order slip boundary condition for gas flow," Phys. Fluids, vol. 19, p. 086101, 2007.

[25] D. Lockerby, J. Reese, D. Emerson and R. Barber, "Velocity boundary condition at solid walls in rarefied gas calculations," Phys Rev E, vol. 70, p. 017303, 2004.

[26] Q. Li, Y. He, G. Tang and W. Tao, "Lattice Boltzmann modeling of microchannel flows in the transition flow regime," Microfluid Nanofluid, vol. 10, pp. 607-618, 2011.

[27] C. Cercignany and S. Lorenzani, "Variational derivation of second-order slip coefficients on the basis of the Boltzmann equation for hard-sphere molecules," Phys Fluids, vol. 22, p. 062004, 2010. 